\title{
Synthesis of Enantioenriched 3,4-Disubstituted Chromans Through Lewis- Base Catalyzed Carbosulfenylation
}

\author{
Travis Menard, Aragorn Laverny and Scott E. Denmark*
}

sdenmark@illinois.edu

Roger Adams Laboratory, Department of Chemistry, University of Illinois, Urbana, IL 61801, USA

SUPPORTING INFORMATION 
TABLE OF CONTENTS

PAGE

Factors Affecting the Reproducibility of the Reaction S3

NMR Spectra and CSP-HPLC/SFC Traces

X-Ray Data

References 


\section{Factors Affecting the Reproducibility of the Reaction}

During the course of surveying the scope of the transformation, several factors were identified which negatively affected the reproducibility of results. The two main factors were the water content of HFIP and reaction times.

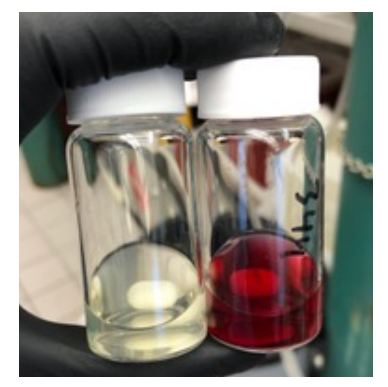

Figure S1. Difference in color between a substrate in dry HFIP (left) vs wet HFIP (right).

To demonstrate the robustness of the reaction, all LB catalyzed reactions were run in nonflame-dried glassware, under air, utilizing bulk solvents. This presented no problems until it was observed that previously tested reactions began to give results significantly worse than previously recorded. In one instance it was noticed that, upon addition of HFIP to the substrate, a significant color change from pale yellow to deep red was observed (Figure 1).

Upon characterization, complete decomposition of the substrate was observed. The HFIP was tested with standard $\mathrm{pH}$ paper and was found to be highly acidic $(\mathrm{pH}=0)$. The HFIP was distilled and stored over $3 \AA \AA$ molecular sieves for one day, followed by retesting of $\mathrm{pH}$. The dry HFIP was found to be $\mathrm{pH}$ neutral. A survey of published literature revealed it had been previously reported the acidity of fluorinated alcoholic solvents increases significantly with increasing mole fraction of water. ${ }^{1}$ Thus it is imperative that dry HFIP be used for the Lewis base catalyzed cyclization to avoid decomposition of the acid-sensitive starting materials.

The issue of reproducibility with respect to reaction times manifested in the increase of minor impurities formed during the reaction. Although no byproducts could be definitively identified, the formation of a number of sulfide and di-sulfide impurities were observed by ${ }^{1} \mathrm{HNMR}$. Despite increases in the conversion of starting materials, the increased challenges of purification lead to decreased yields with longer reaction times. To alleviate this issue, reaction times were standardized to 24 hours which provided a good balance of starting material conversion and reaction cleanliness. For substrates featuring strongly electron-withdrawing groups on A-ring, the catalyst loading could be increased from $10 \%$ to $20 \%$ resulting in slight increases in yield. This 
was deemed unnecessary for general reaction conditions as the increase in yield was mostly negligible relative to the increased catalyst loading. 
(E)-(Cinnamyloxy)benzene (1a) $-{ }^{1} \mathrm{H}$ NMR (500 MHz, $\left.\mathrm{CDCl}_{3}\right)$

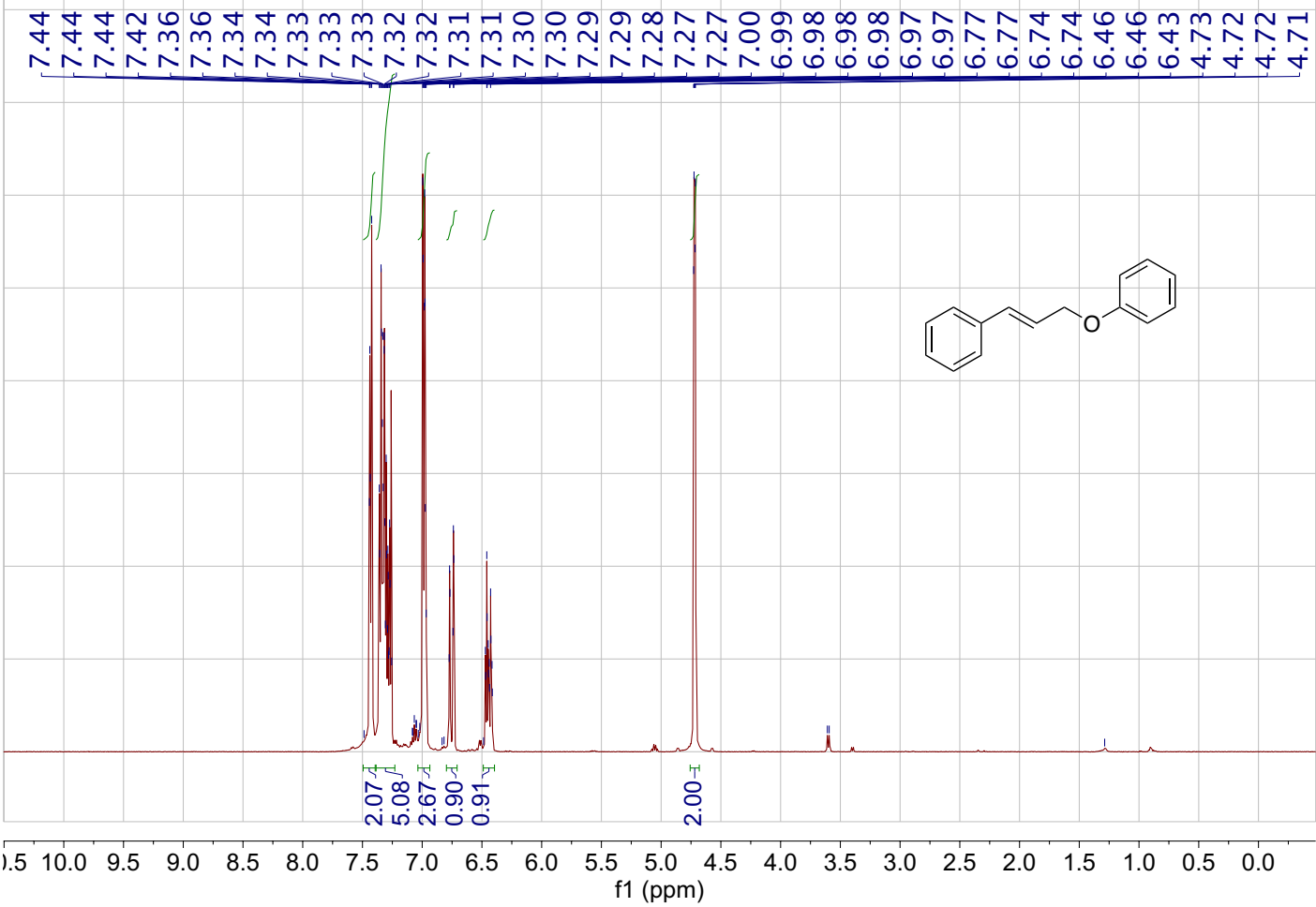

(E)-1-(Cinnamyloxy)-4-methoxybenzene (1c) - ${ }^{1} \mathrm{H}$ NMR $\left(500 \mathrm{MHz}, \mathrm{CDCl}_{3}\right)$

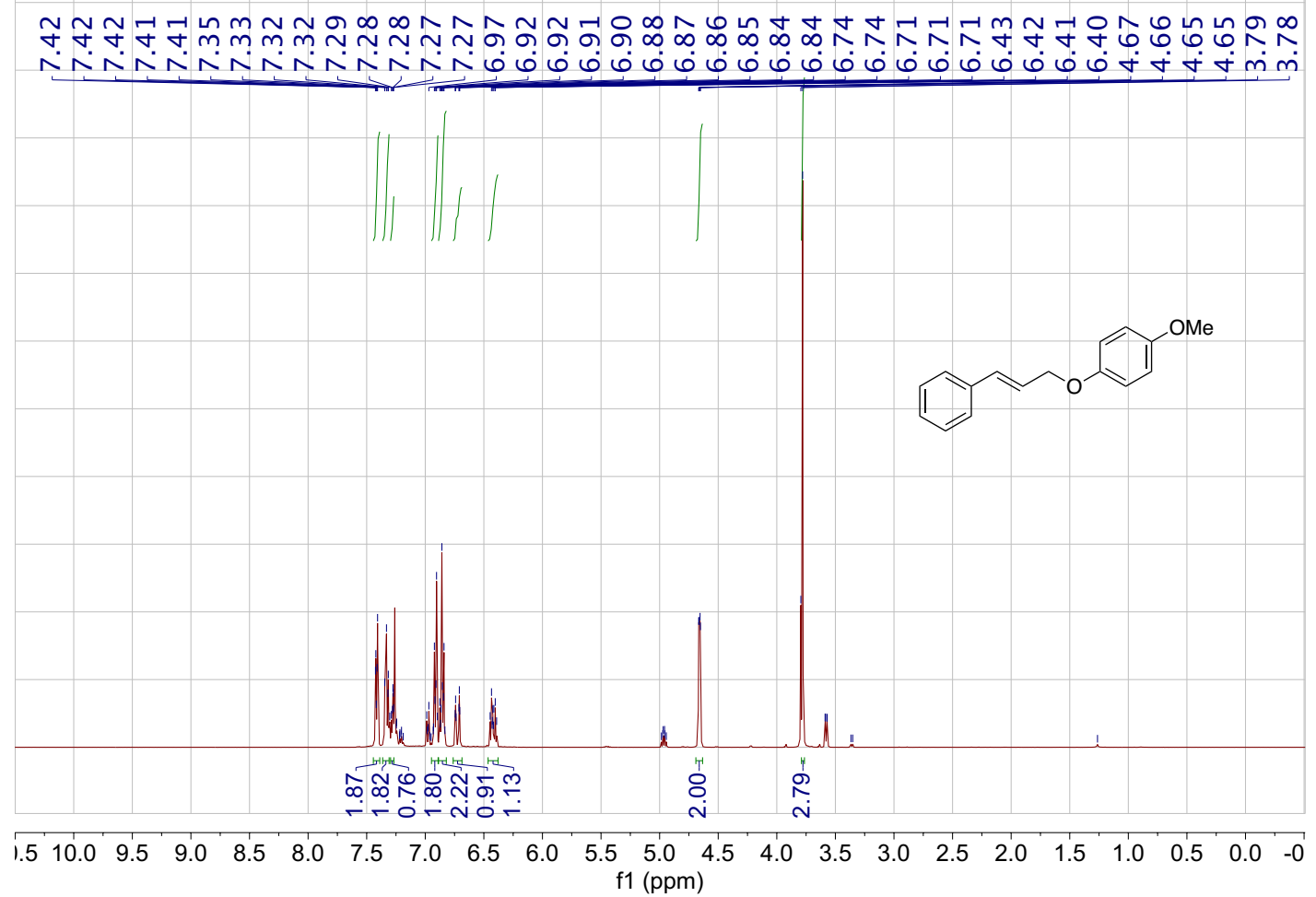


(E)-1-(Cinnamyloxy)-2-methoxybenzene (1d) - ${ }^{1} \mathrm{H}$ NMR $\left(500 \mathrm{MHz}, \mathrm{CDCl}_{3}\right)$

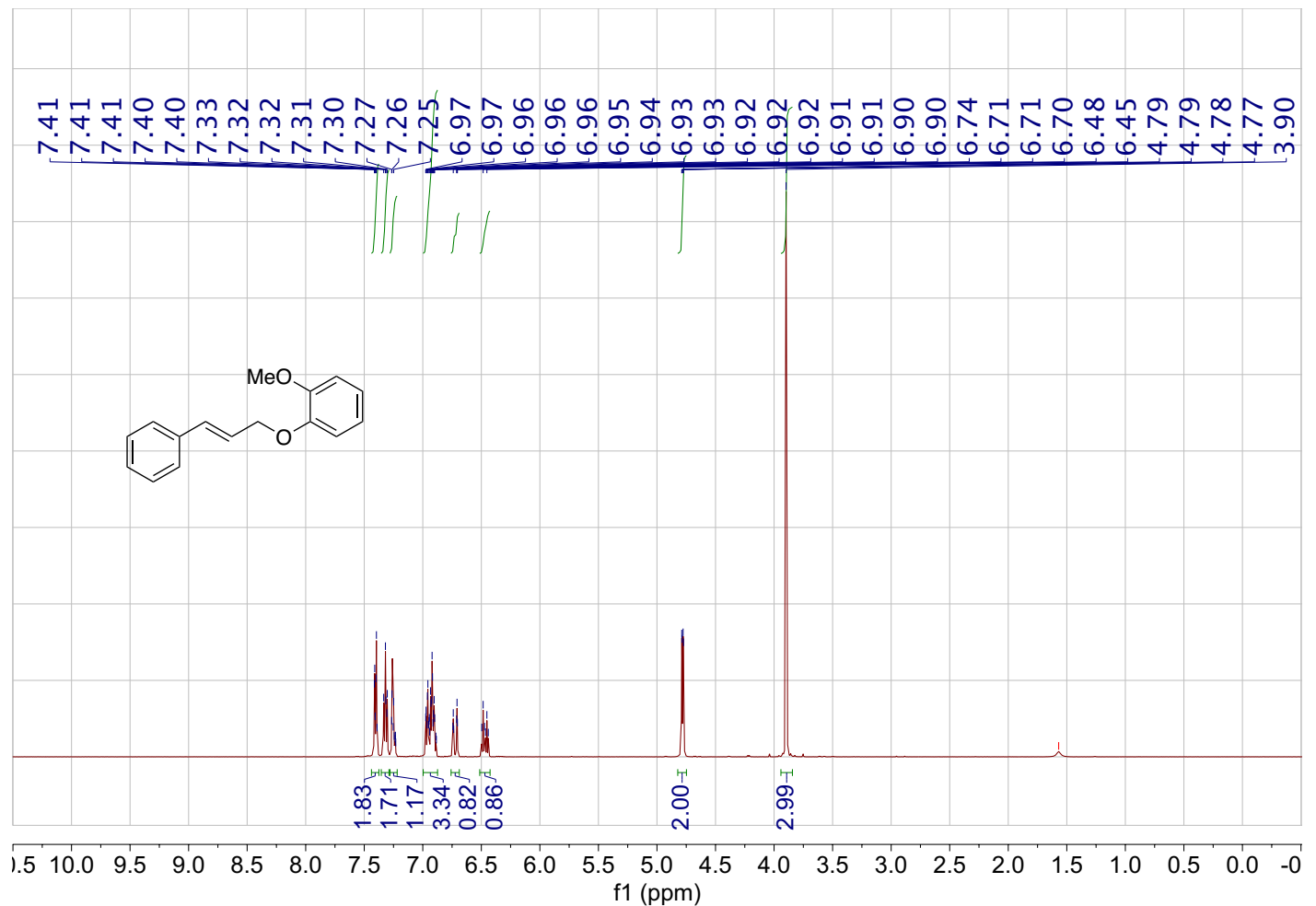

\section{(E)-1-Bromo-4-(cinnamyloxy)benzene (1e) - ${ }^{1} \mathrm{H}$ NMR $\left(500 \mathrm{MHz}, \mathrm{CDCl}_{3}\right)$}

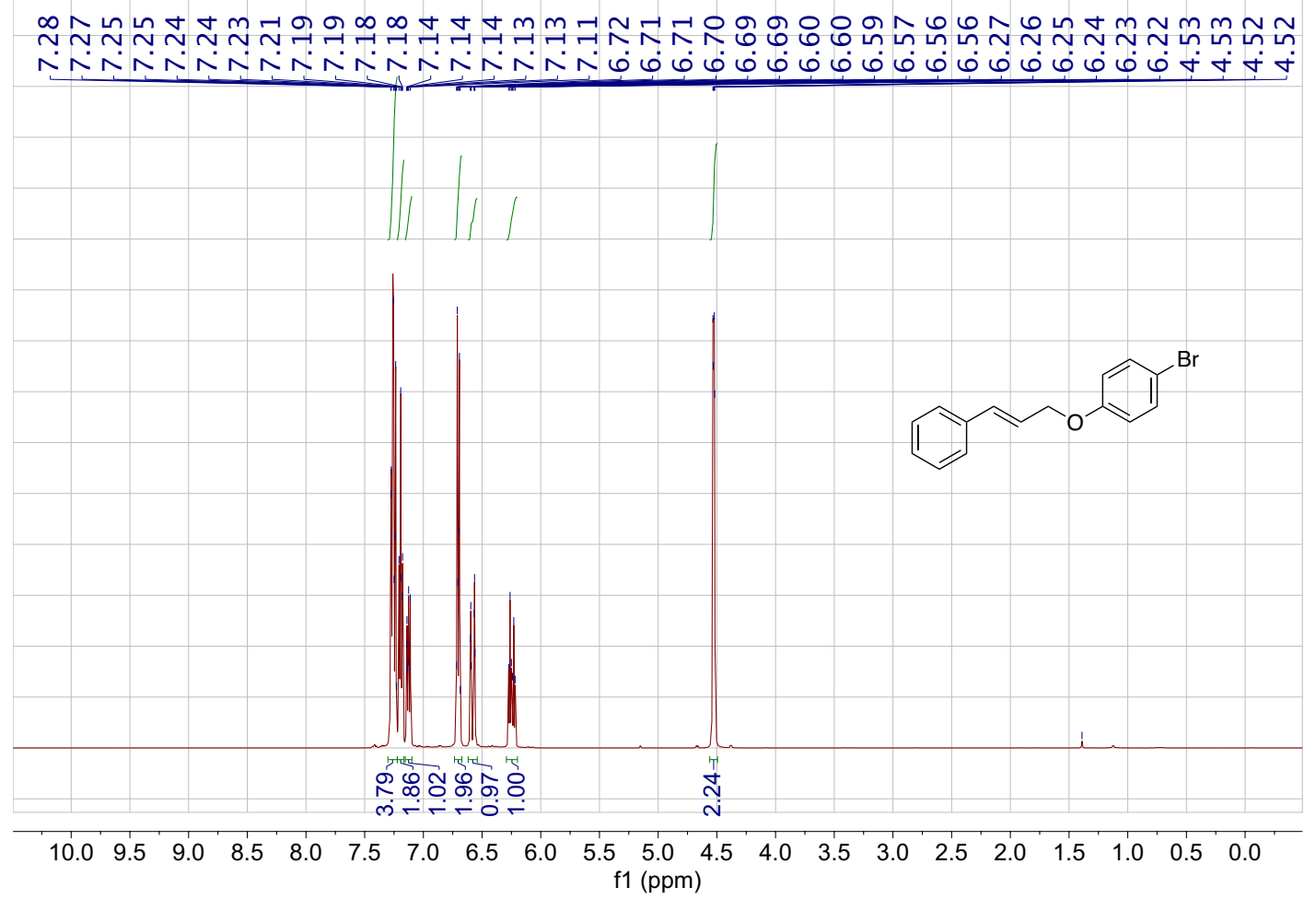


(E)-1-(Cinnamyloxy)-4-(trifluoromethyl)benzene (1g) - ${ }^{1} \mathrm{H}$ NMR $\left(500 \mathrm{MHz}, \mathrm{CDCl}_{3}\right)$

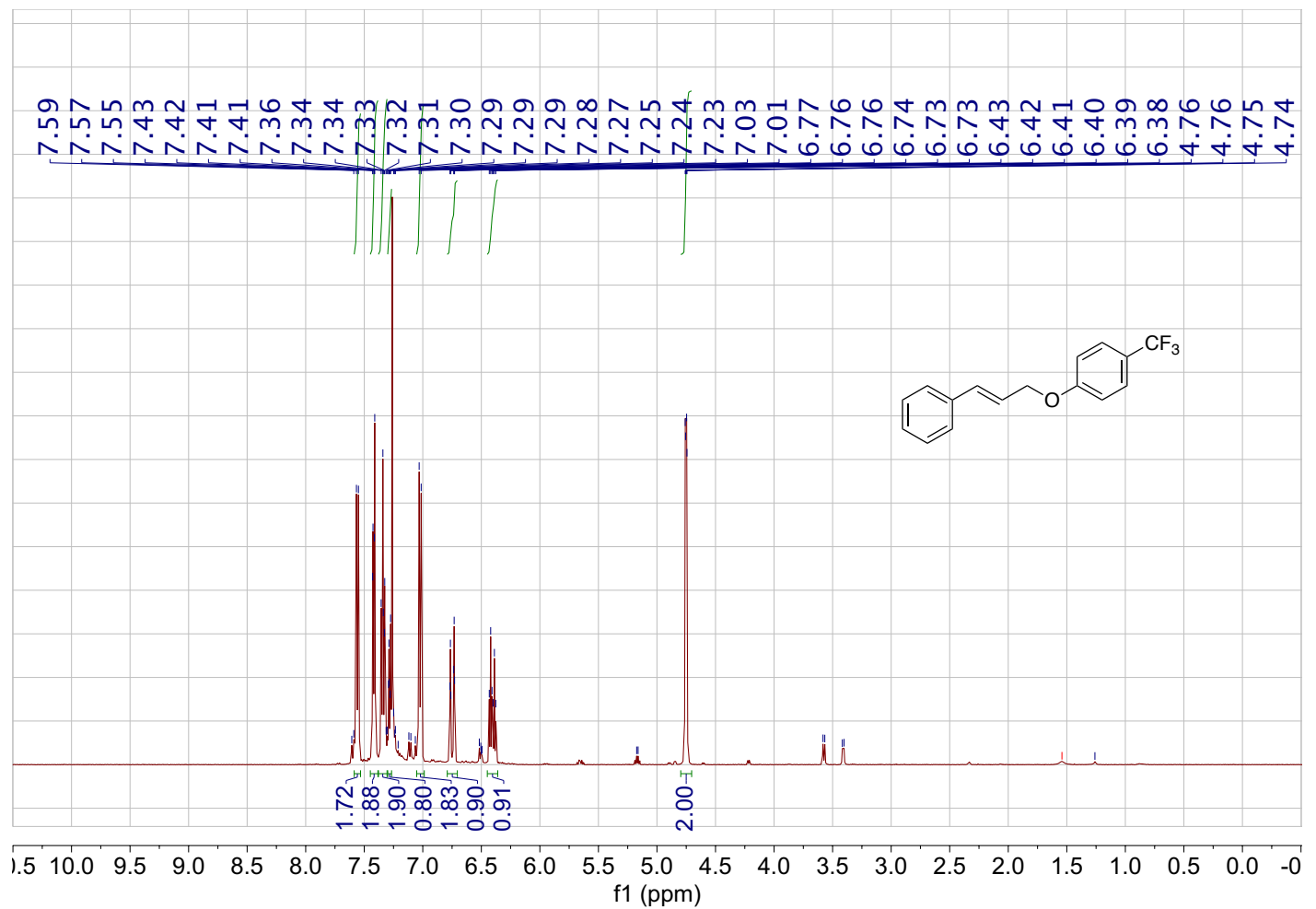

(E)-1-(Cinnamyloxy)-3-methoxybenzene (1j) - ${ }^{1} \mathrm{H}$ NMR $\left(500 \mathrm{MHz}, \mathrm{CDCl}_{3}\right)$

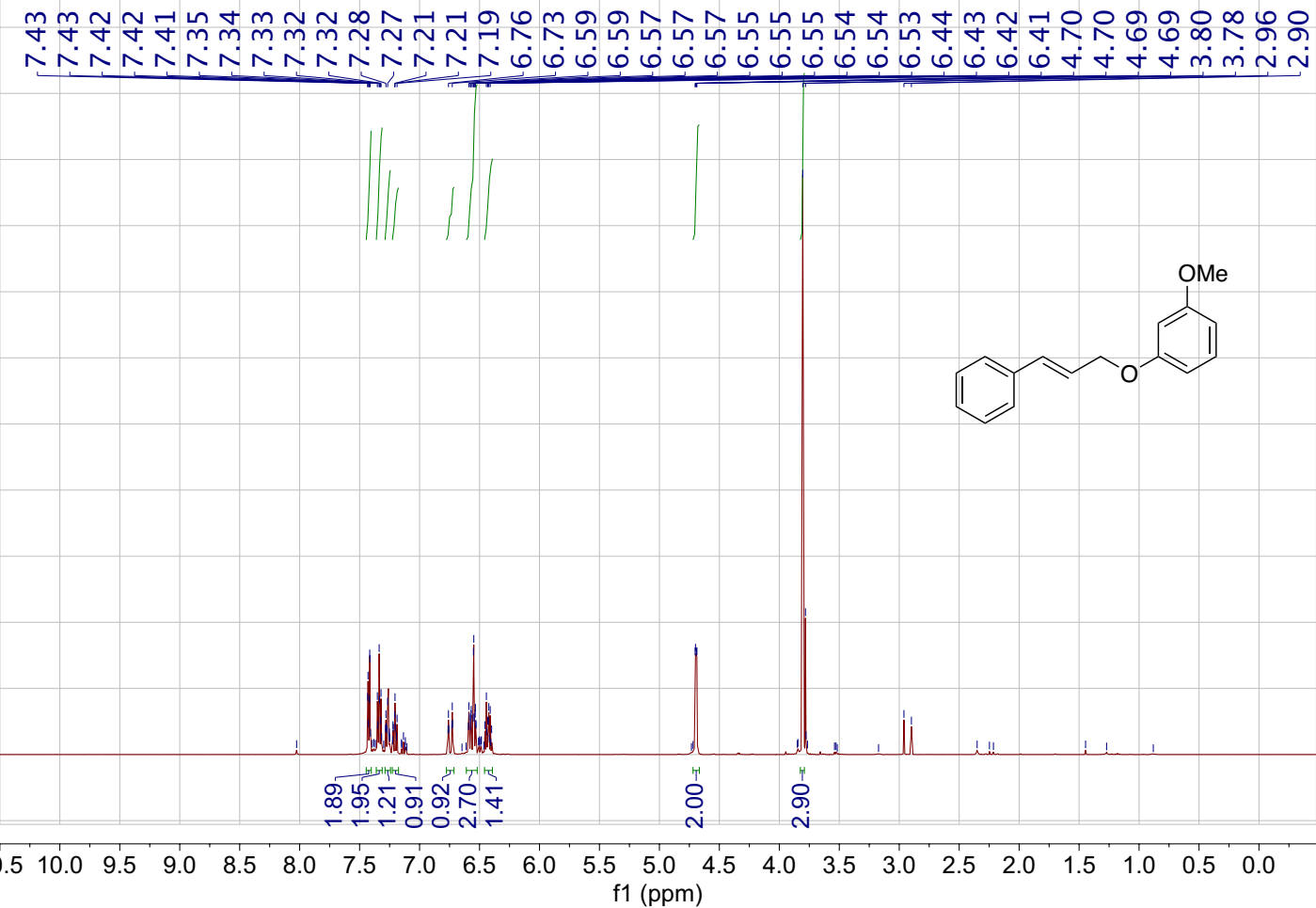


(E)-1-Chloro-4-(3-phenoxyprop-1-en-1-yl)benzene (1n) - ${ }^{1} \mathrm{H}$ NMR (500 MHz, $\mathrm{CDCl}_{3}$ )

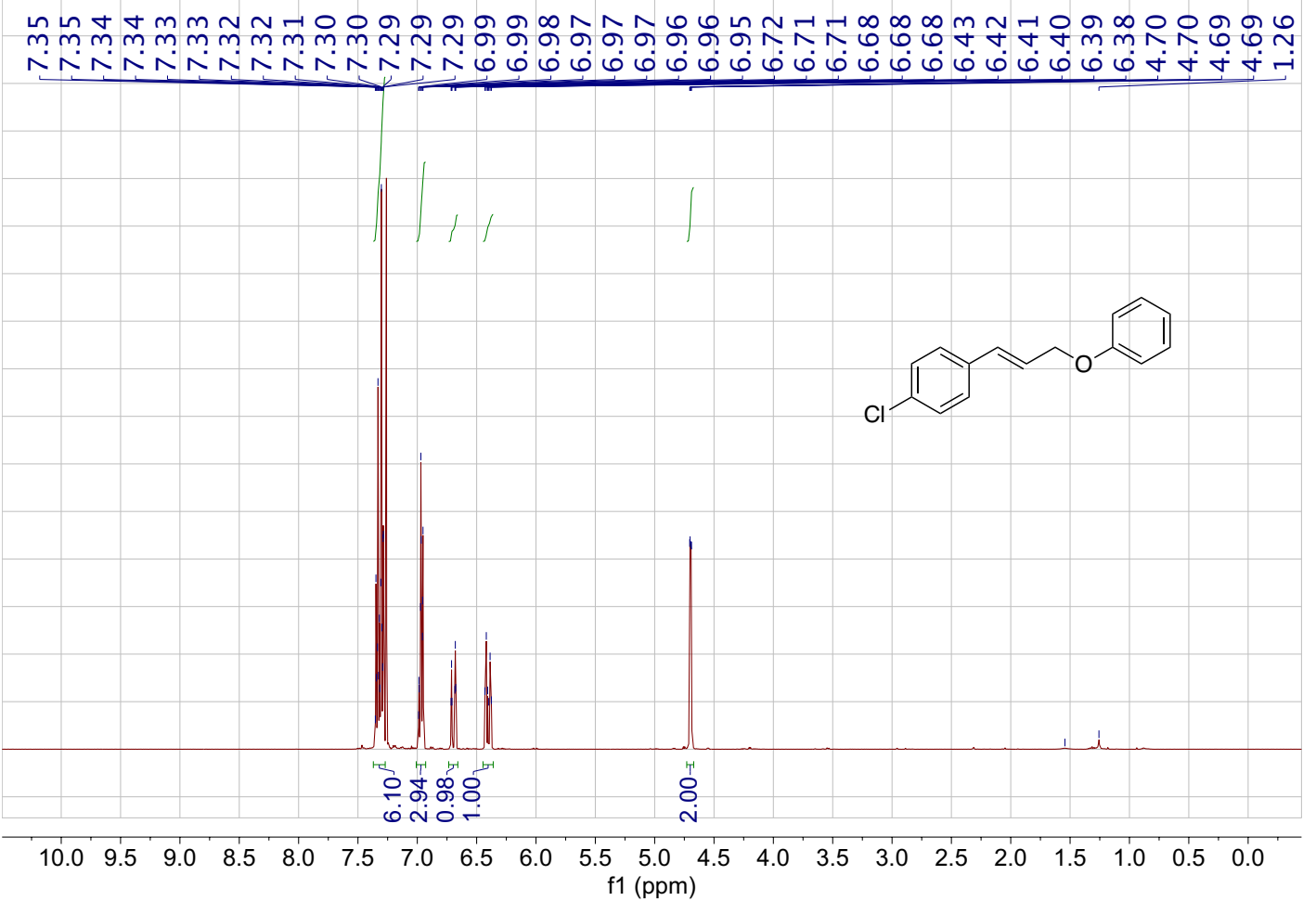

(E)-1-Methoxy-4-(3-phenoxyprop-1-en-1-yl)benzene (1o) - ${ }^{1} \mathrm{H}$ NMR $\left(500 \mathrm{MHz}, \mathrm{CDCl}_{3}\right)$

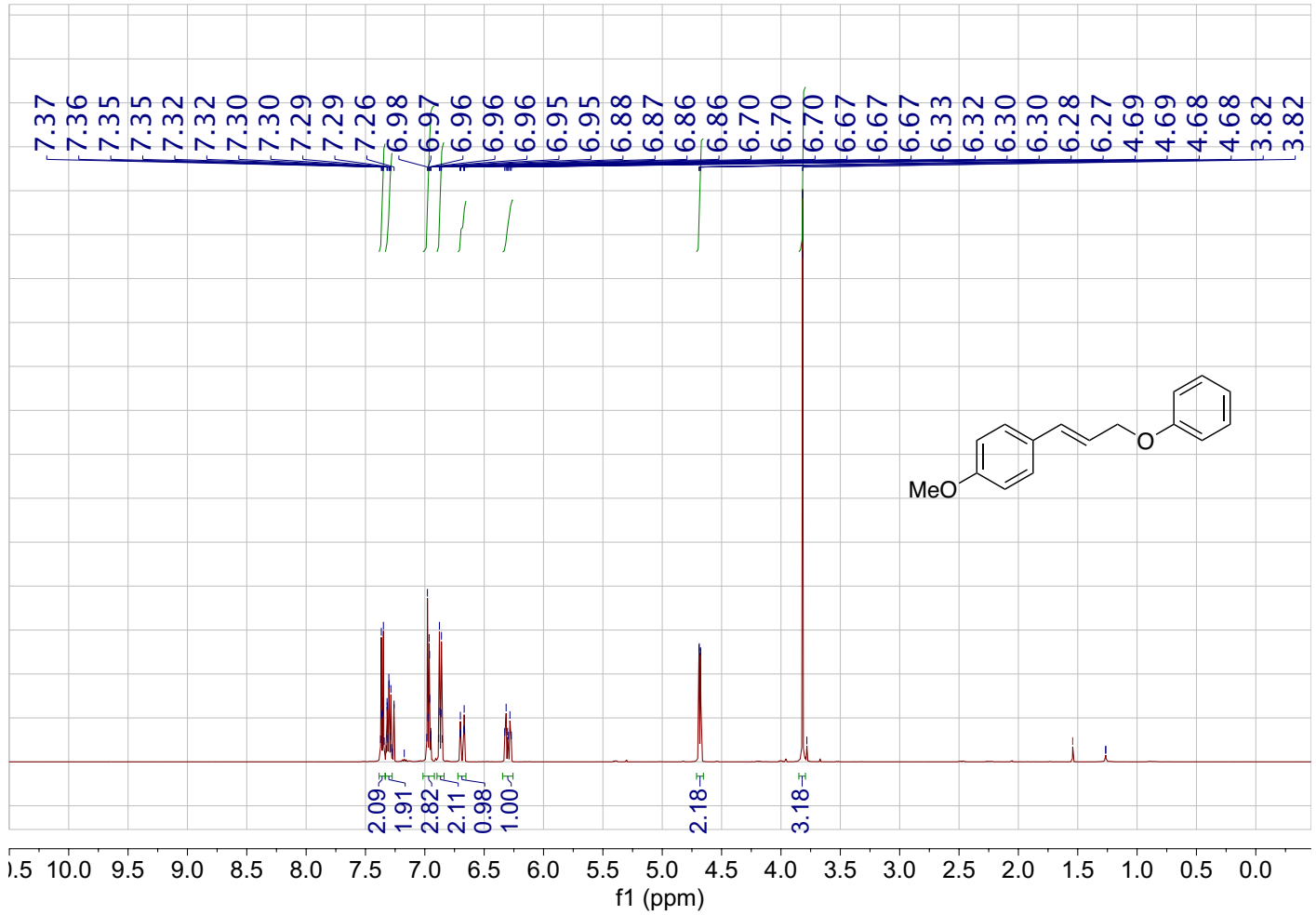


(E)-1-Bromo-4-(3-phenoxyprop-1-en-1-yl)benzene (1p”) - ${ }^{1} \mathrm{H}$ NMR (500 MHz, $\left.\mathrm{CDCl}_{3}\right)$

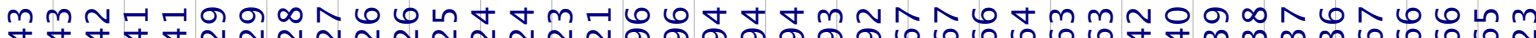

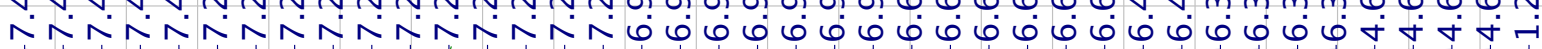

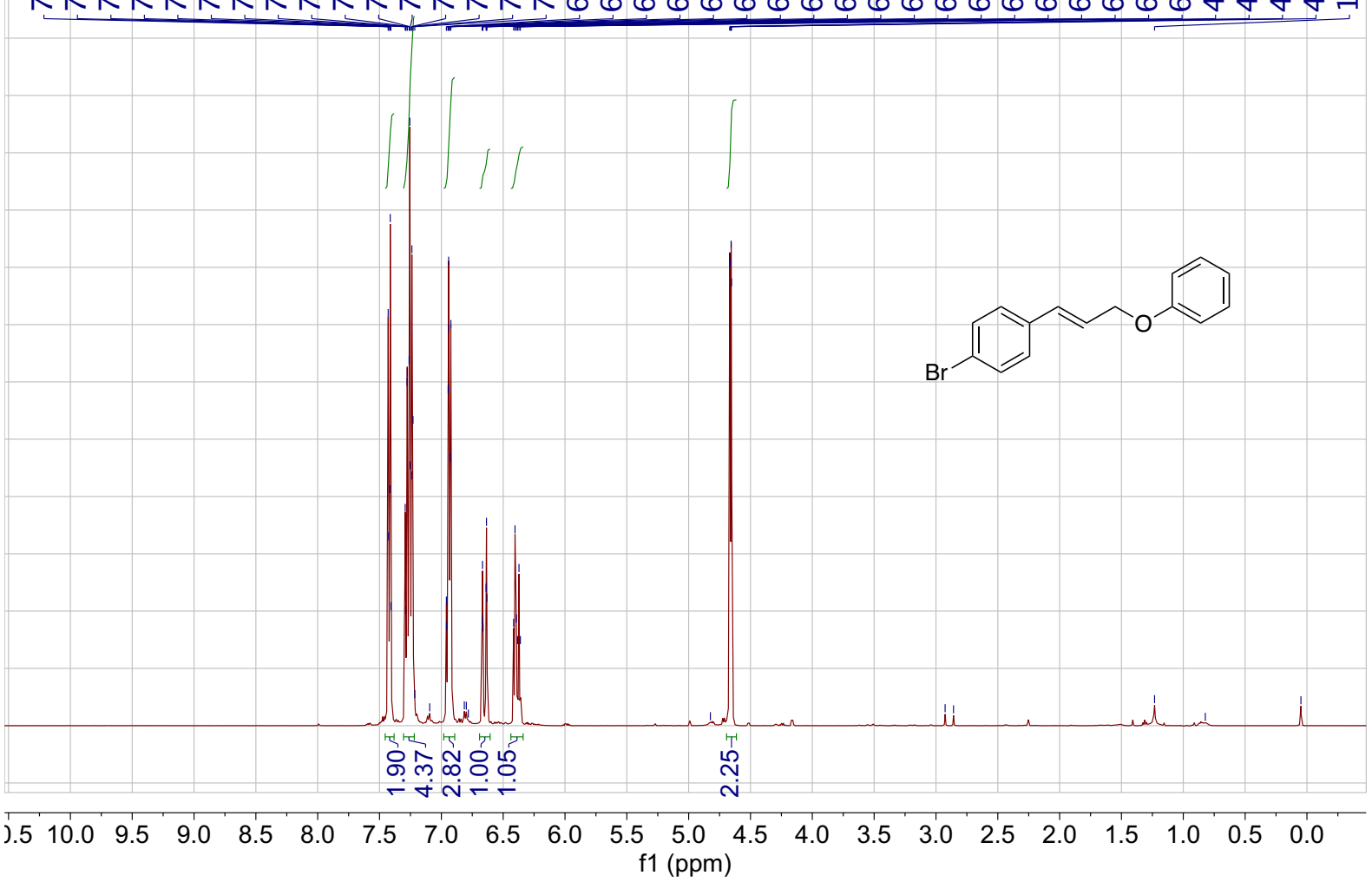


(E)-1-(Cinnamyloxy)-2-fluorobenzene (1f) - ${ }^{1} \mathrm{H}$ NMR $\left(500 \mathrm{MHz}, \mathrm{CDCl}_{3}\right) ;{ }^{13} \mathrm{C}\left\{{ }^{1} \mathrm{H}\right\}(126 \mathrm{MHz}$, $\left.\mathrm{CDCl}_{3}\right)$

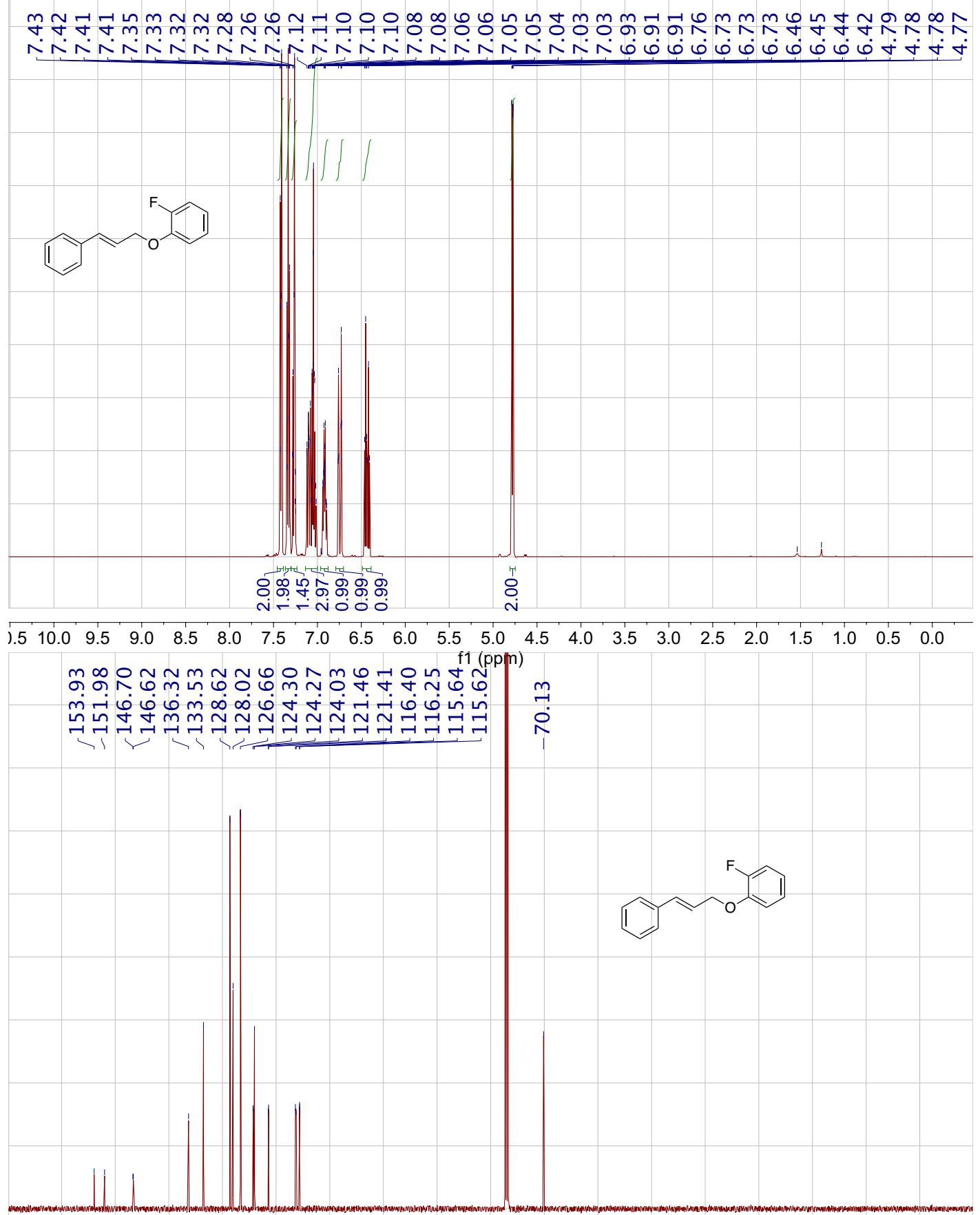

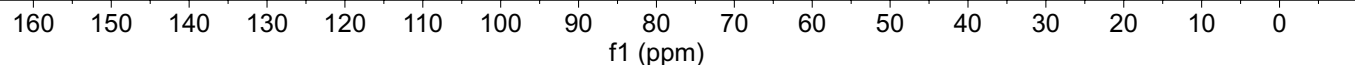


(E)-1-Ethyl 4-(Cinnamyloxy)benzoate (1h) - ${ }^{1} \mathrm{H}$ NMR $\left(500 \mathrm{MHz}, \mathrm{CDCl}_{3}\right) ;{ }^{13} \mathrm{C}\left\{{ }^{1} \mathrm{H}\right\}(126 \mathrm{MHz}$, $\left.\mathrm{CDCl}_{3}\right)$
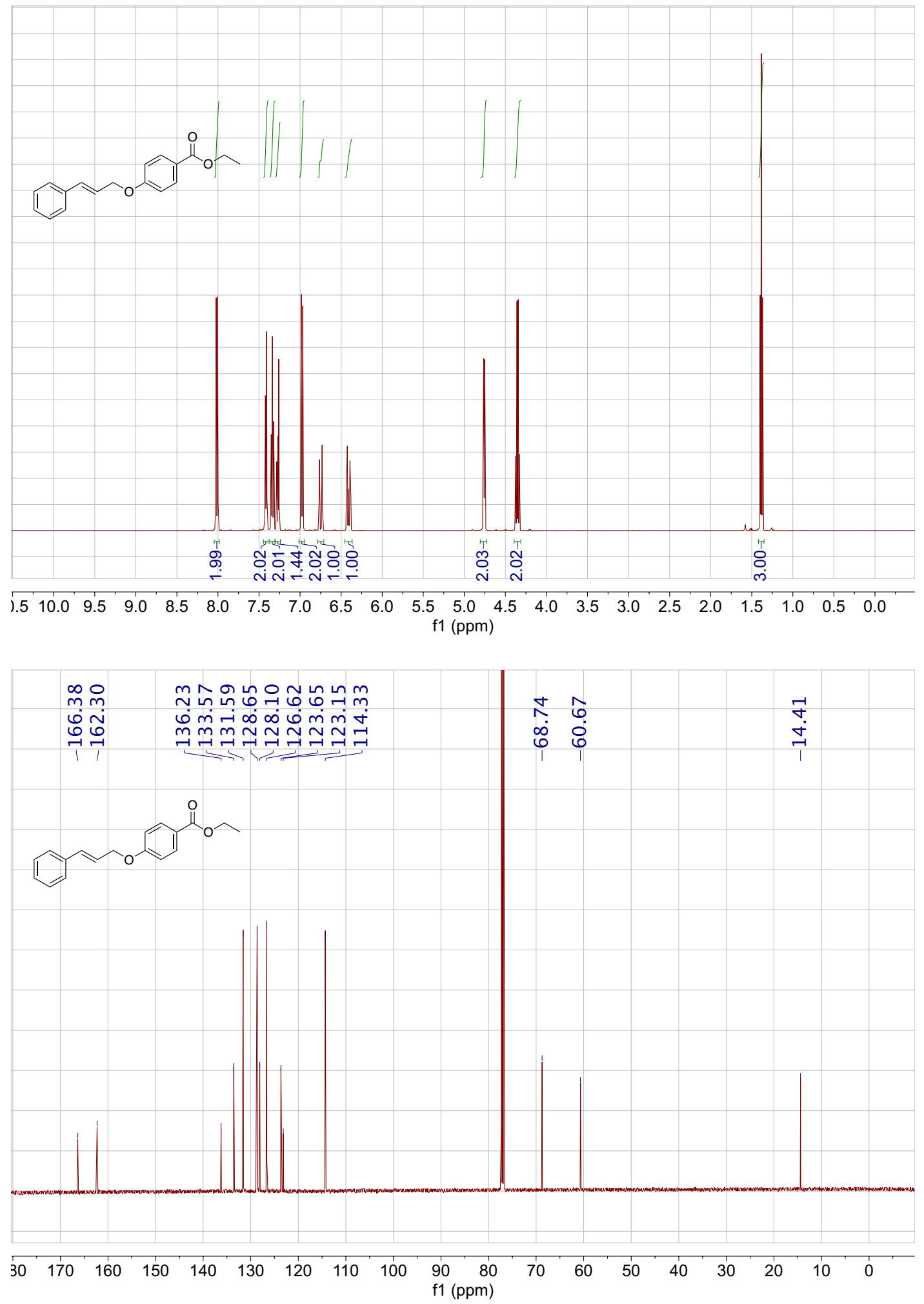
(E)-N-(4-(Cinnamyloxy)phenyl)acetamide (1i) - ${ }^{1} \mathrm{H}$ NMR (500 MHz, $\left.\mathrm{CDCl}_{3}\right) ;{ }^{13} \mathrm{C}\left\{{ }^{1} \mathrm{H}\right\}(126$ $\left.\mathrm{MHz}, \mathrm{CDCl}_{3}\right)$
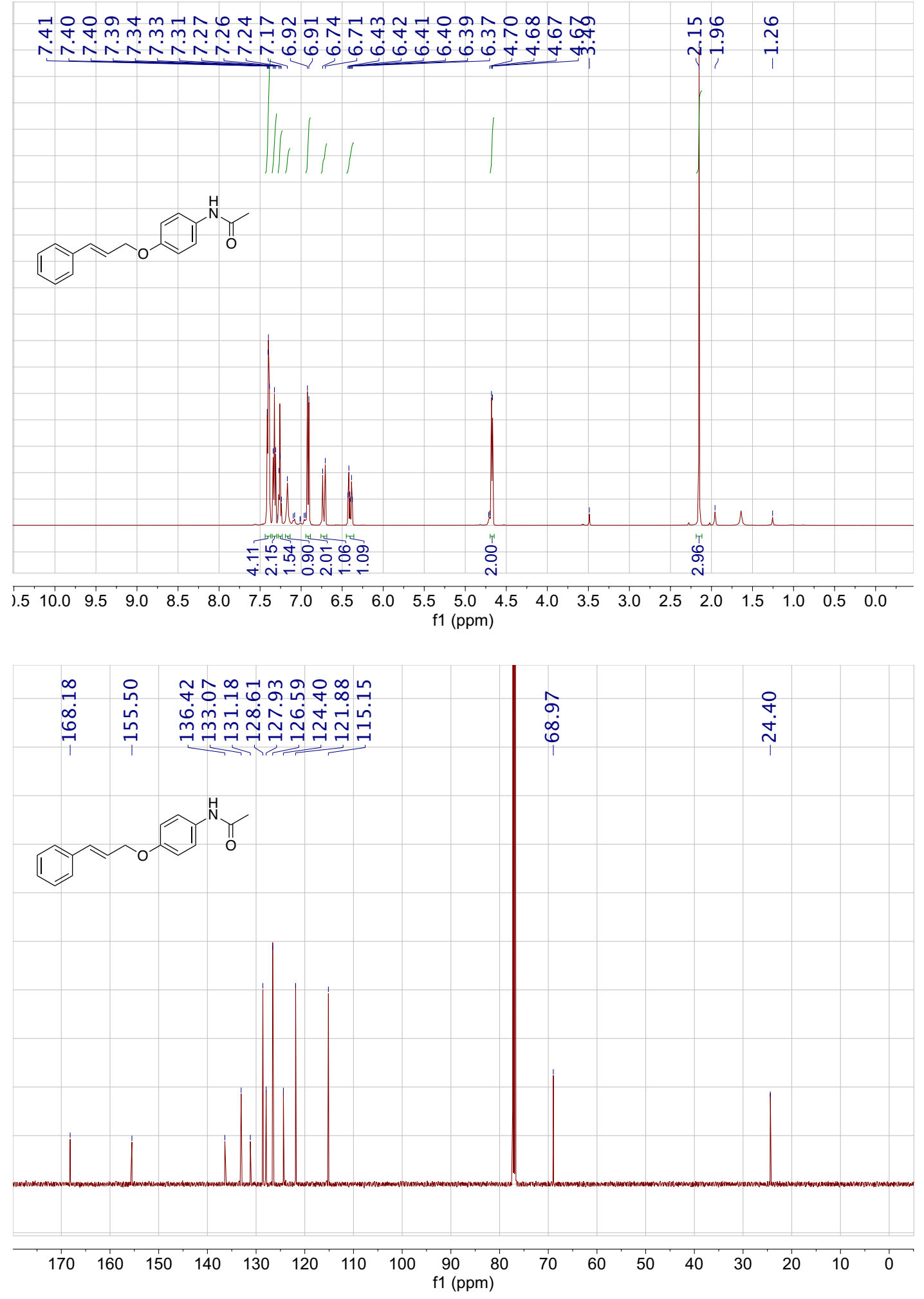
(E)-1-(Cinnamyloxy)-3-nitrobenzene (1k) $-{ }^{1} \mathrm{H}$ NMR $\left(500 \mathrm{MHz}, \mathrm{CDCl}_{3}\right) ;{ }^{13} \mathrm{C}\left\{{ }^{1} \mathrm{H}\right\}(126 \mathrm{MHz}$, $\left.\mathrm{CDCl}_{3}\right)$
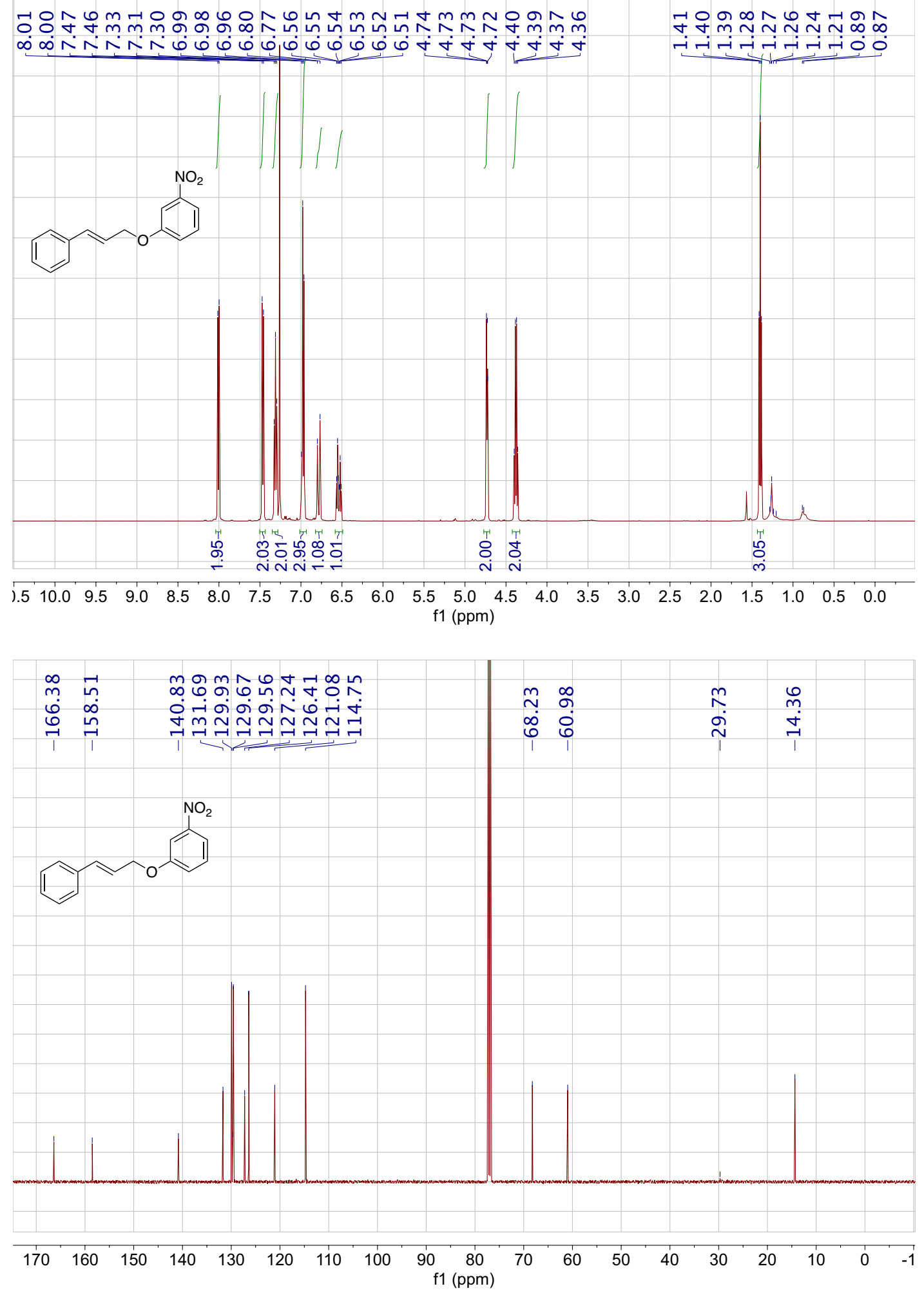
tert-Butyl (E)-3-(3-hydroxyprop-1-en-1-yl)-1H-indole-1-carboxylate (D6) - ${ }^{1} \mathrm{H}$ NMR (500 MHz, $\left.\mathrm{CDCl}_{3}\right)$

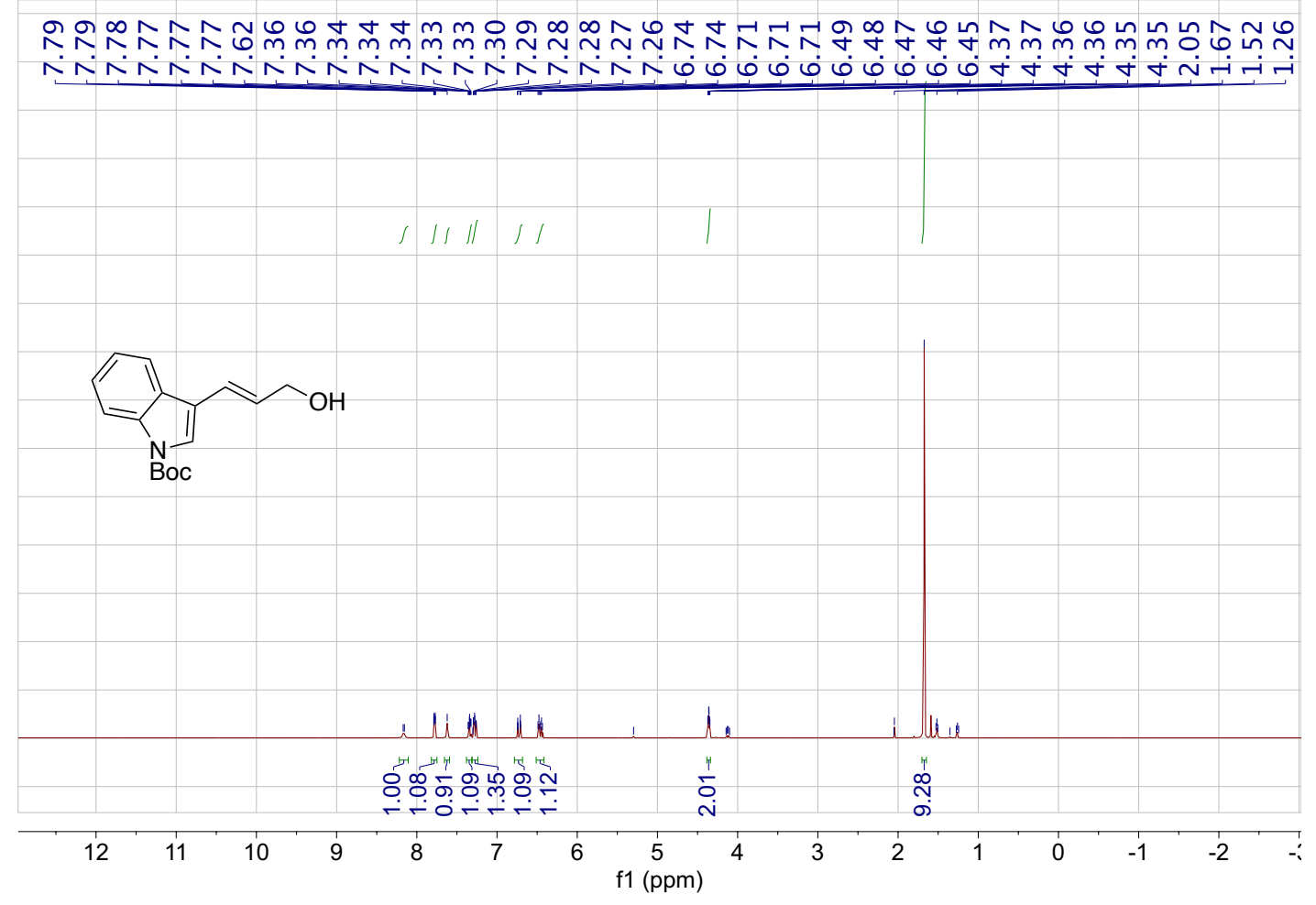


tert-Butyl (E)-3-(3-Bromoprop-1-en-1-yl)-1H-indole-1-carboxylate (E6) - ${ }^{1} \mathrm{H}$ NMR (500 MHz, $\left.\mathrm{CDCl}_{3}\right)$

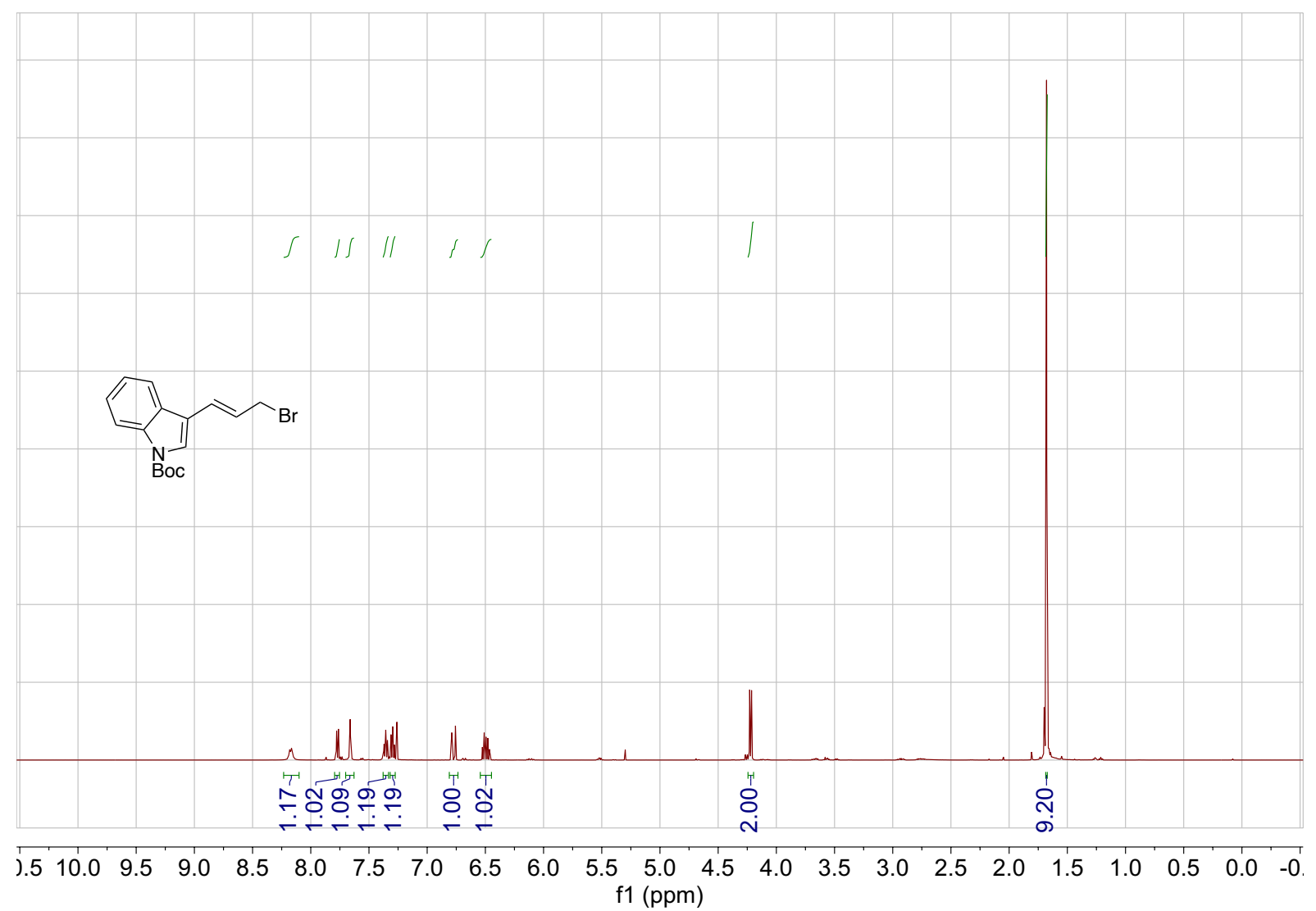


(E)-1-Bromo-2-(3-phenoxyprop-1-en-1-yl)benzene (11) - ${ }^{1} \mathrm{H} \mathrm{NMR}\left(500 \mathrm{MHz}, \mathrm{CDCl}_{3}\right) ;{ }^{13} \mathrm{C}\left\{{ }^{1} \mathrm{H}\right\}$ $\left(126 \mathrm{MHz}, \mathrm{CDCl}_{3}\right)$
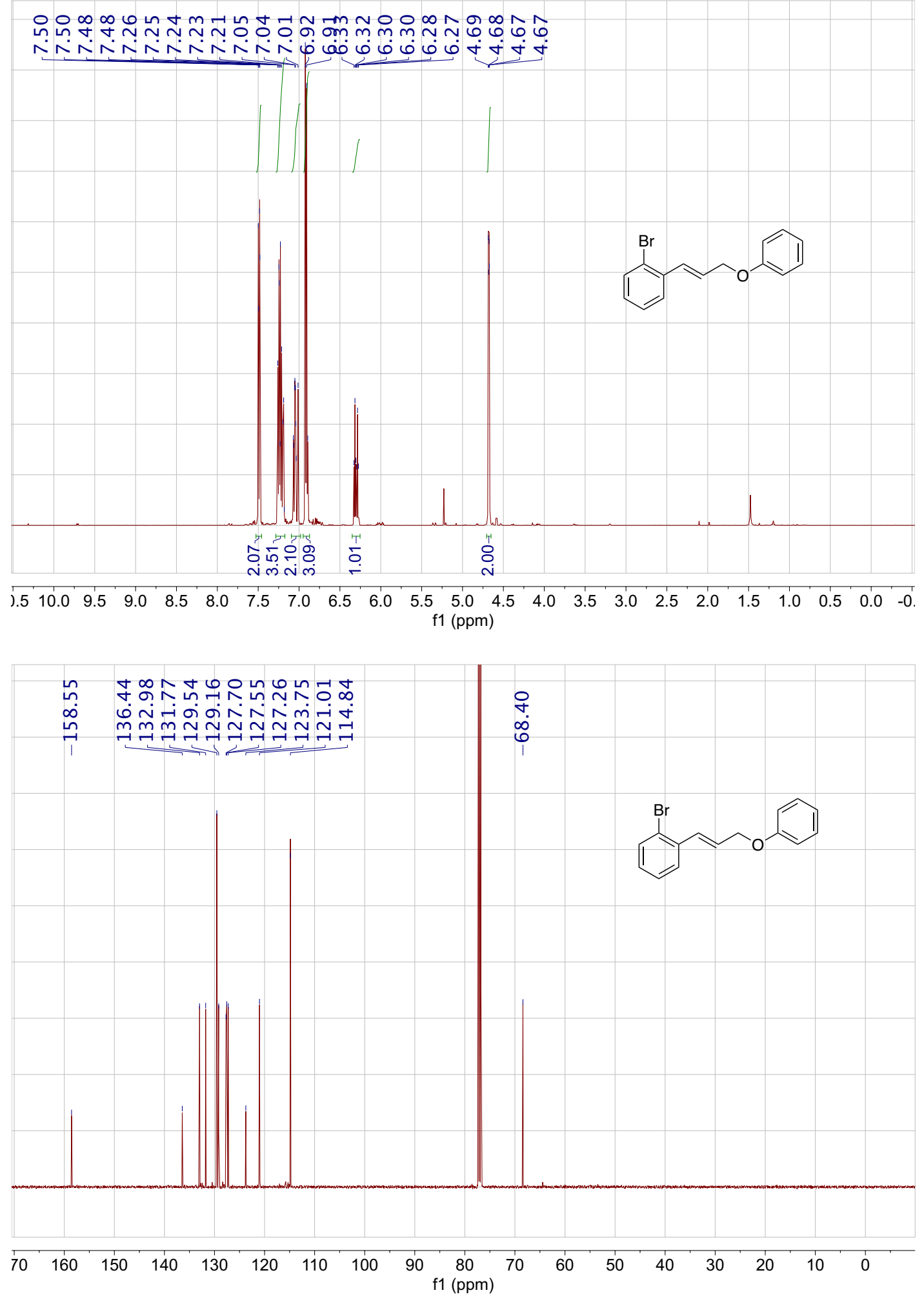
(E)-1-(Benzyloxy)-4-(3-phenoxyprop-1-en-1-yl)benzene (1 m) - ${ }^{1} \mathrm{H}$ NMR $\left(500 \mathrm{MHz}, \mathrm{CDCl}_{3}\right)$; ${ }^{13} \mathrm{C}\left\{{ }^{1} \mathrm{H}\right\}\left(126 \mathrm{MHz}, \mathrm{CDCl}_{3}\right)$

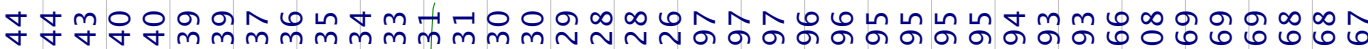

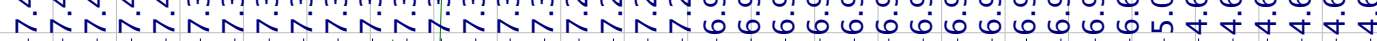
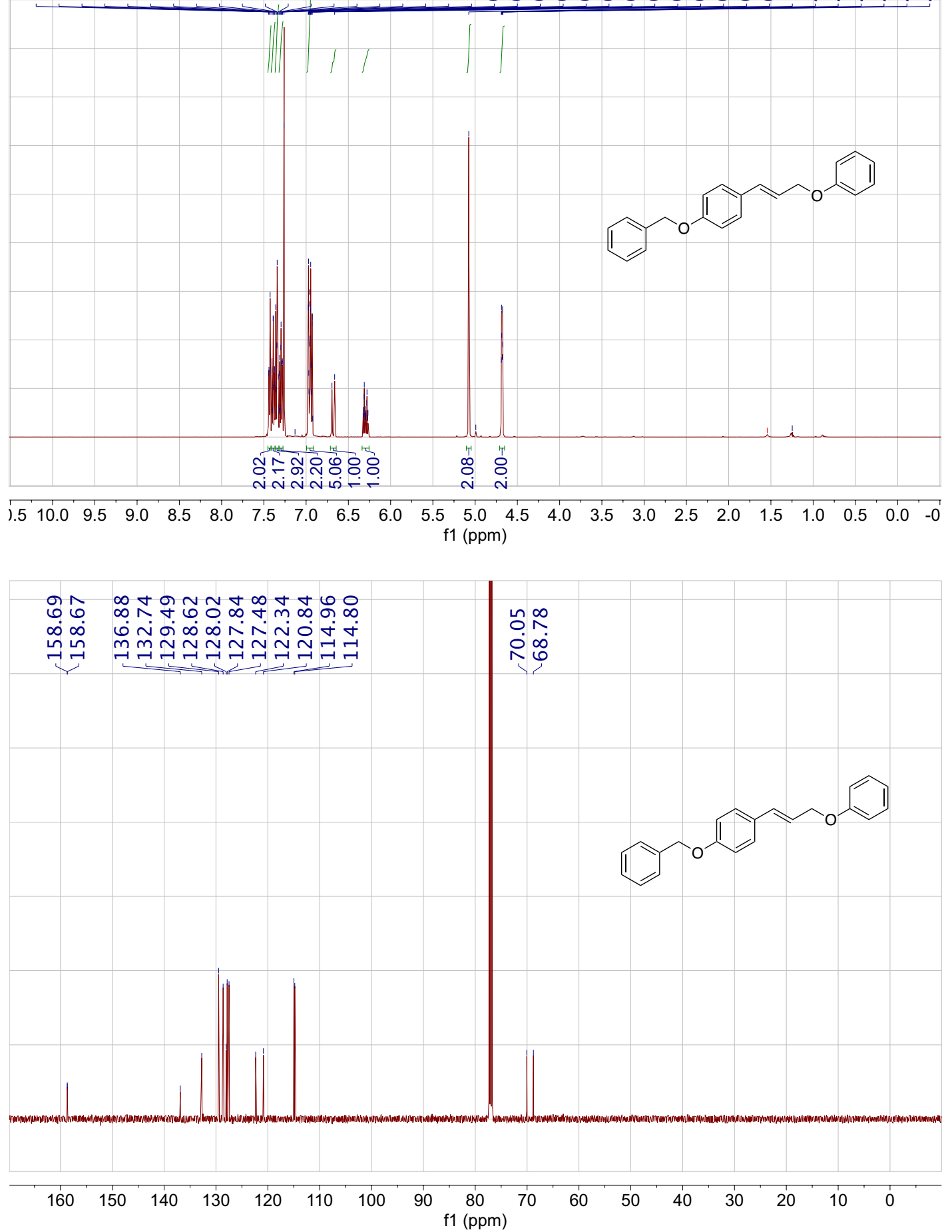
tert-Butyl (E)-3-(3-Phenoxyprop-1-en-1-yl)-1H-indole-1-carboxylate (1q) - ${ }^{1} \mathrm{H}$ NMR (500 MHz, $\left.\mathrm{CDCl}_{3}\right)$

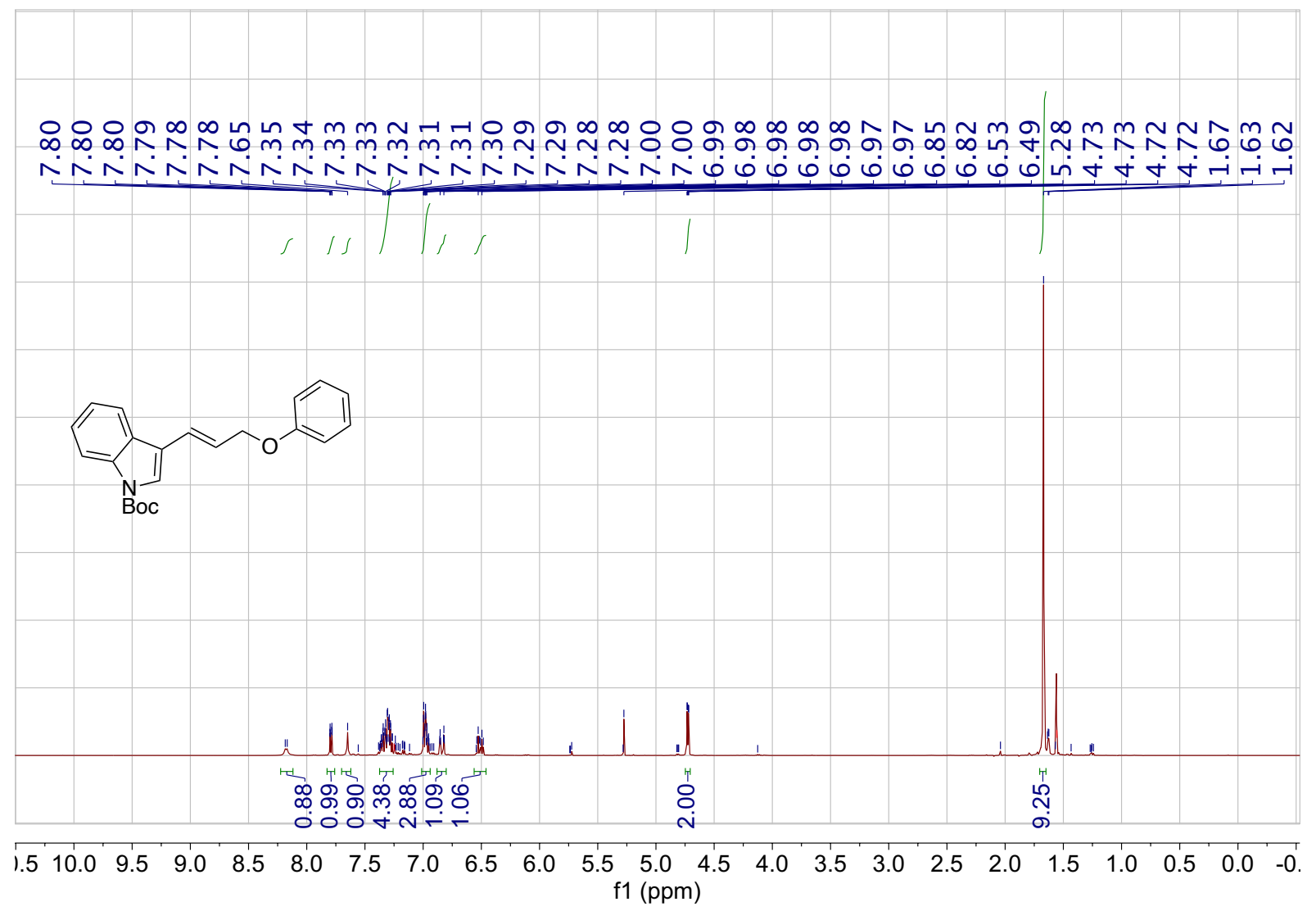


Ethyl (E)-4-(3-phenoxyprop-1-en-1-yl)benzoate (1p) - ${ }^{1} \mathrm{H}$ NMR $\left(500 \mathrm{MHz}, \mathrm{CDCl}_{3}\right) ;{ }^{13} \mathrm{C}\left\{{ }^{1} \mathrm{H}\right\}$ $\left(126 \mathrm{MHz}, \mathrm{CDCl}_{3}\right)$
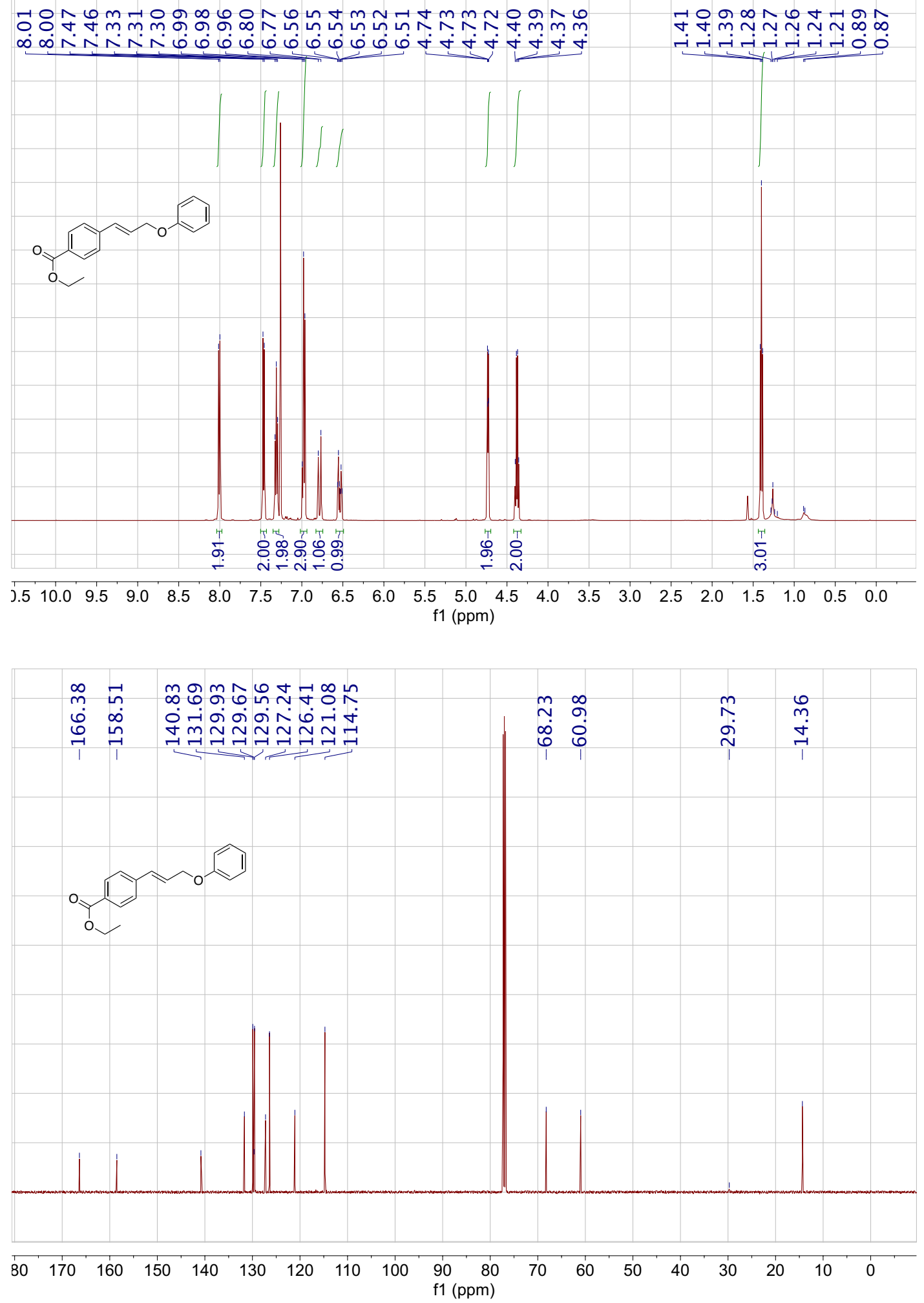
(E)-((3-Cyclohexylallyl)oxy)benzene (1r) - ${ }^{1} \mathrm{H}$ NMR $\left(500 \mathrm{MHz}, \mathrm{CDCl}_{3}\right) ;{ }^{13} \mathrm{C}\left\{{ }^{1} \mathrm{H}\right\}(126 \mathrm{MHz}$, $\left.\mathrm{CDCl}_{3}\right)$
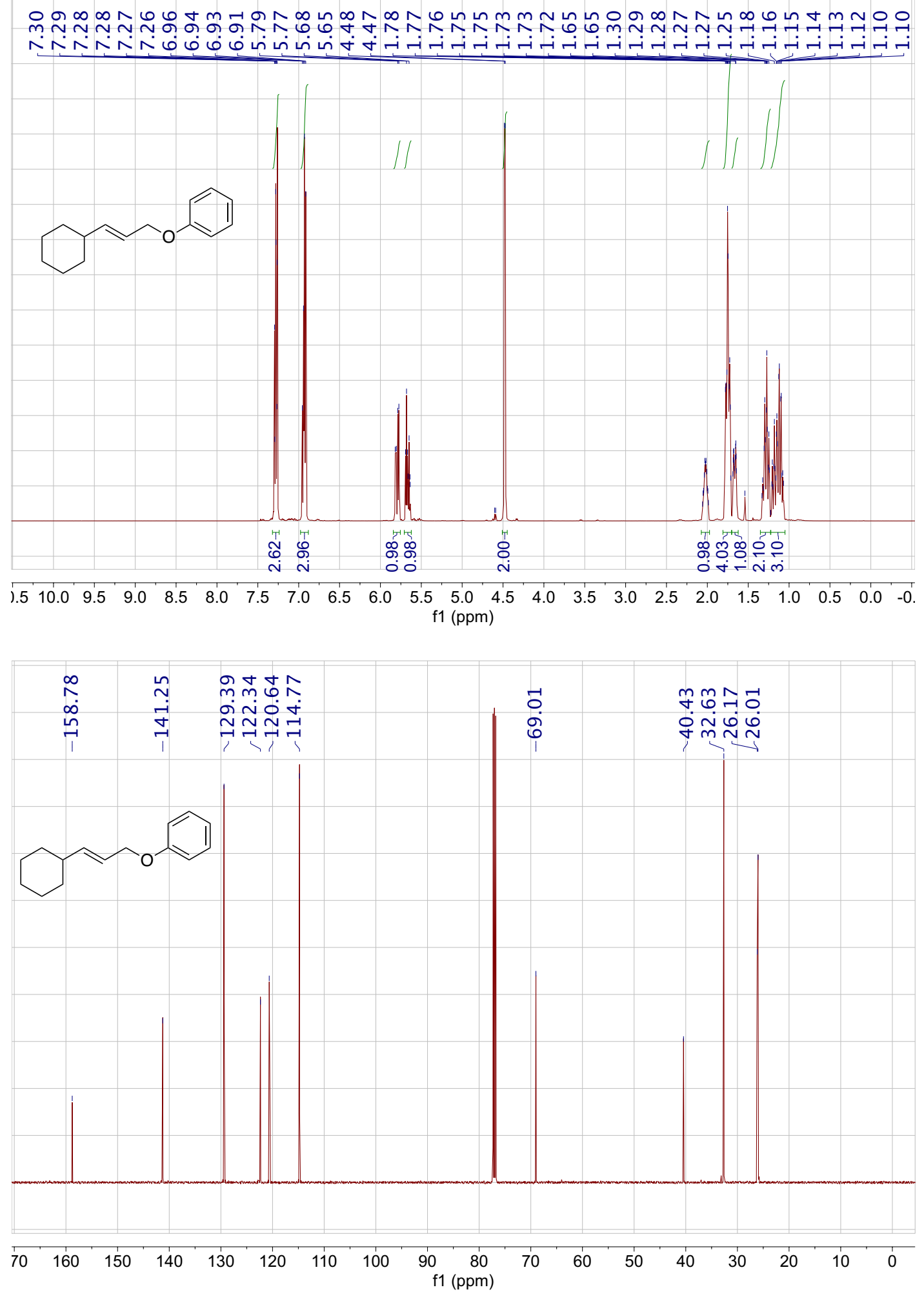
(3R,4S)-3-((2,6-Diisopropylphenyl)thio)-4-phenylchromane - (3a) - ${ }^{1} \mathrm{H}$ NMR $(500 \mathrm{MHz}$, $\left.\mathrm{CDCl}_{3}\right) ;{ }^{13} \mathrm{C}\left\{{ }^{1} \mathrm{H}\right\}\left(126 \mathrm{MHz}, \mathrm{CDCl}_{3}\right)$
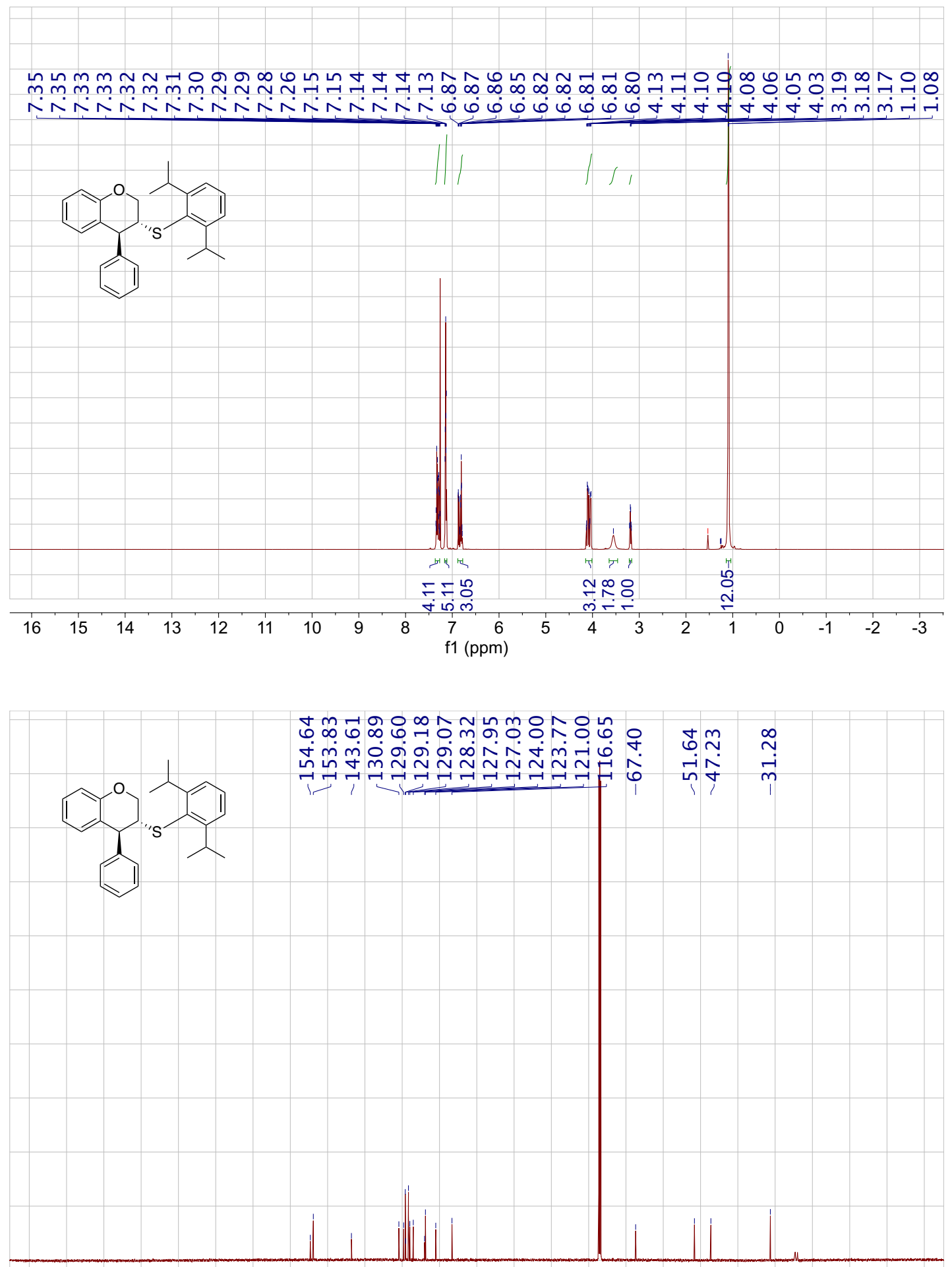

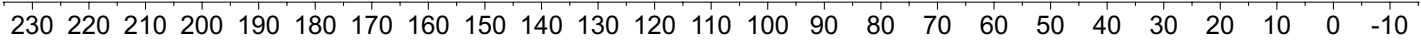
f1 (ppm) 

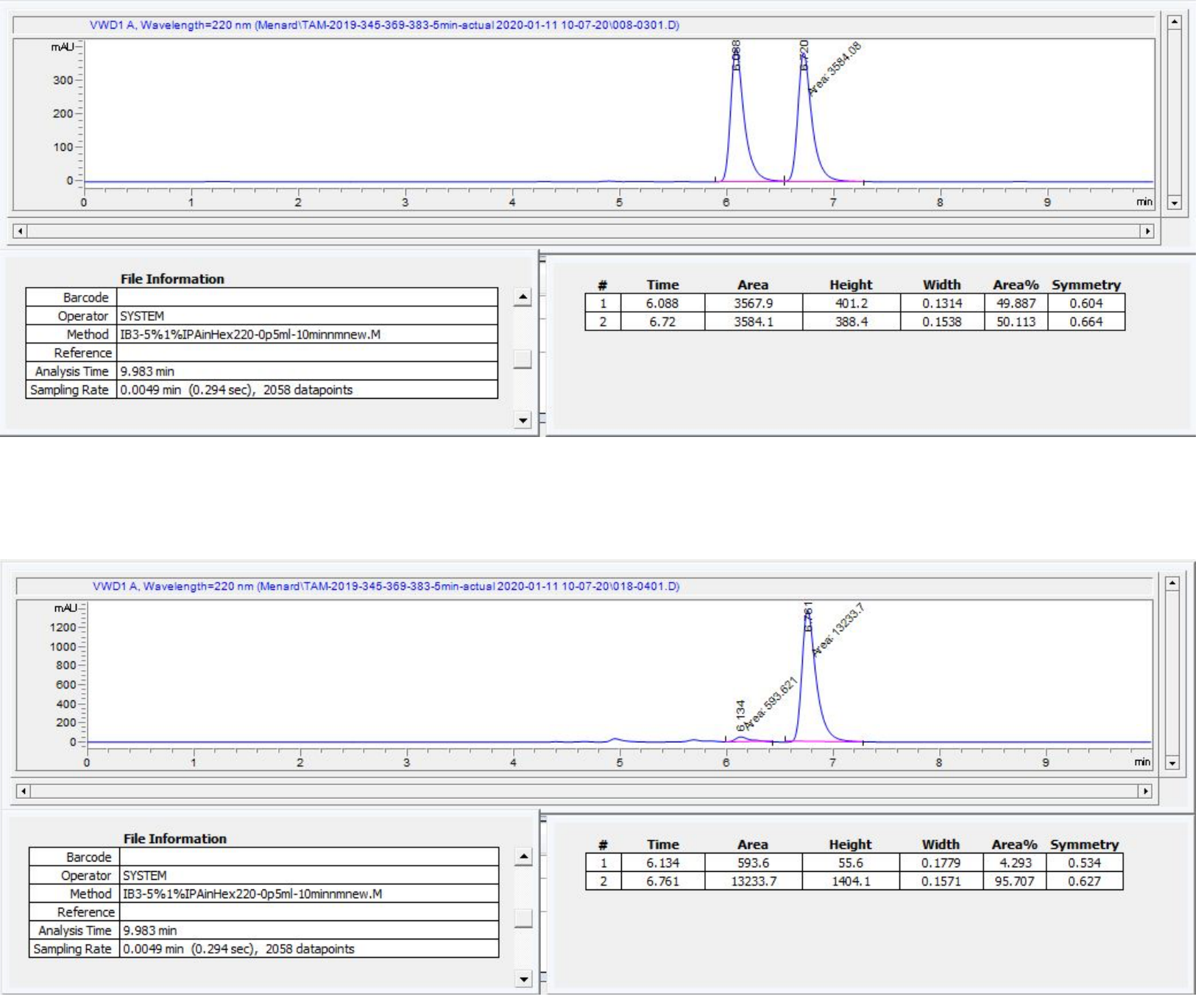
$(3 R, 4 S)-3-((2,6-D i i s o p r o p y l p h e n y l) t h i o)-6-m e t h y l-4-p h e n y l c h r o m a n e-(3 \mathbf{b})-{ }^{1} \mathrm{H}$ NMR (500 $\left.\mathrm{MHz}, \mathrm{CDCl}_{3}\right) ;{ }^{13} \mathrm{C}\left\{{ }^{1} \mathrm{H}\right\}\left(126 \mathrm{MHz}, \mathrm{CDCl}_{3}\right)$
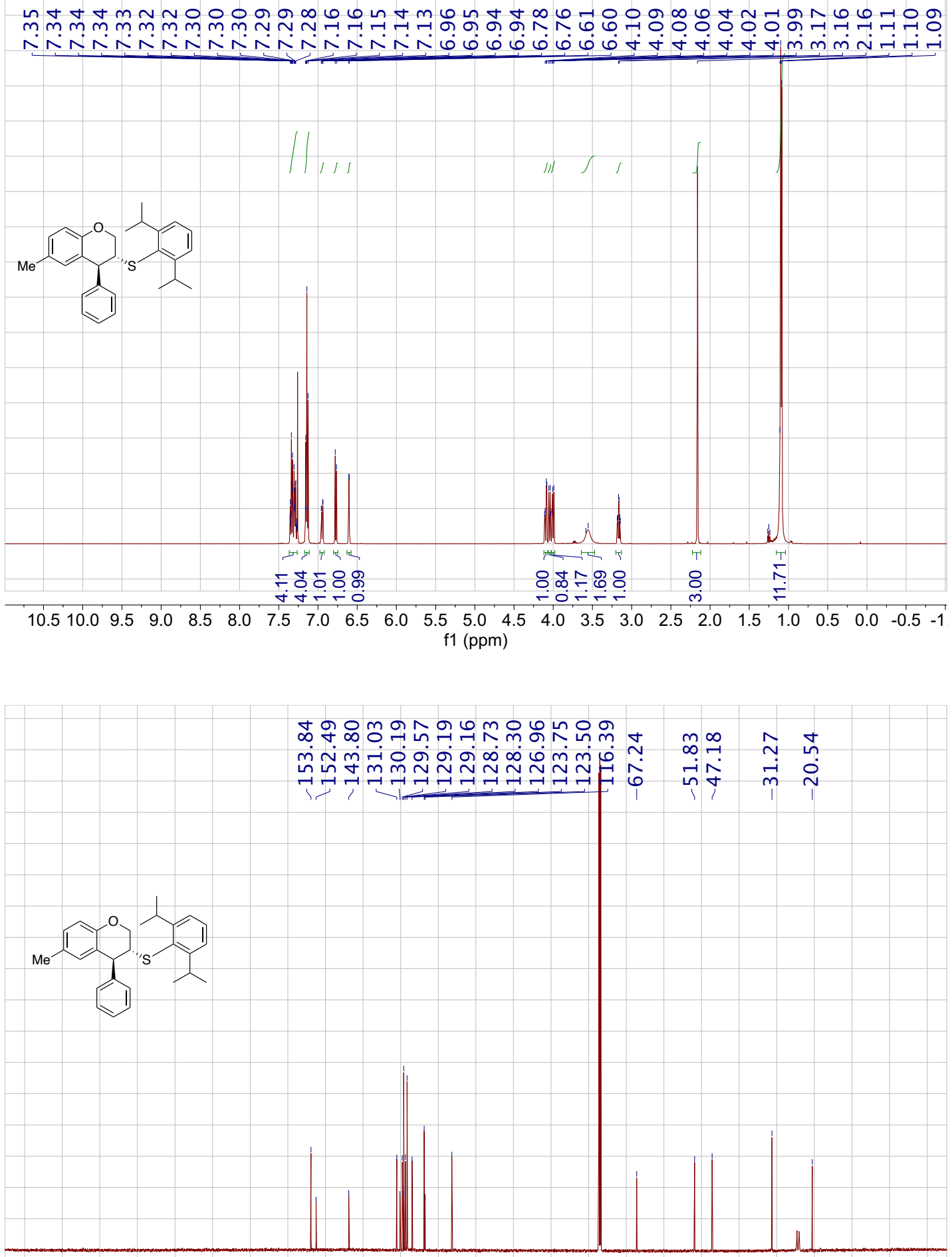

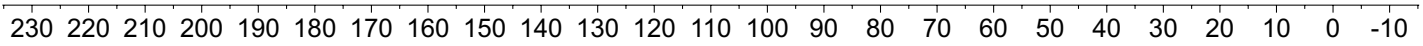
f1 (ppm) 

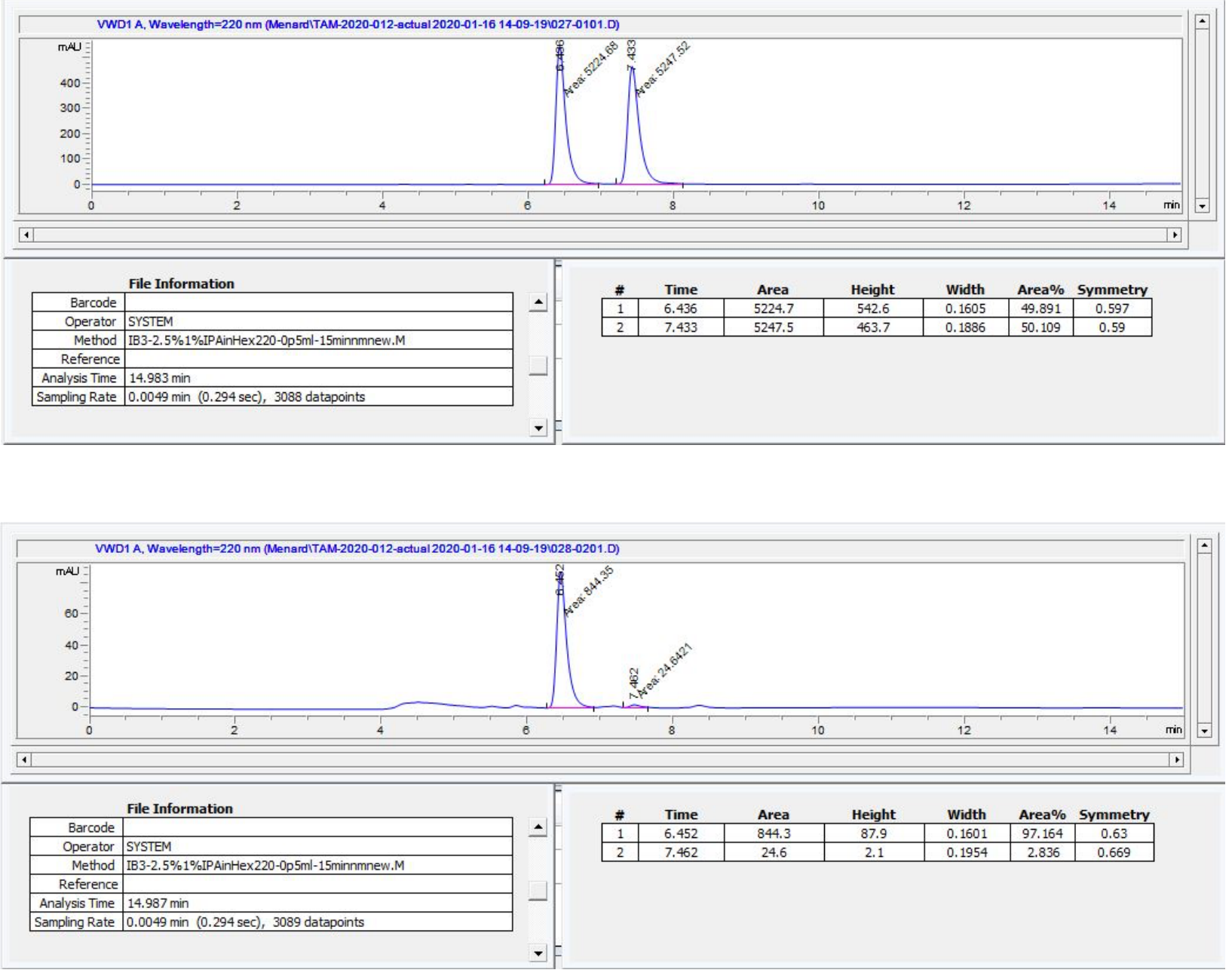
(3R,4S)-3-((2,6-Diisopropylphenyl)thio)-6-methoxy-4-phenylchromane - (3c) - ${ }^{1} \mathrm{H}$ NMR (500 $\left.\mathrm{MHz}, \mathrm{CDCl}_{3}\right) ;{ }^{13} \mathrm{C}\left\{{ }^{1} \mathrm{H}\right\}\left(126 \mathrm{MHz}, \mathrm{CDCl}_{3}\right)$
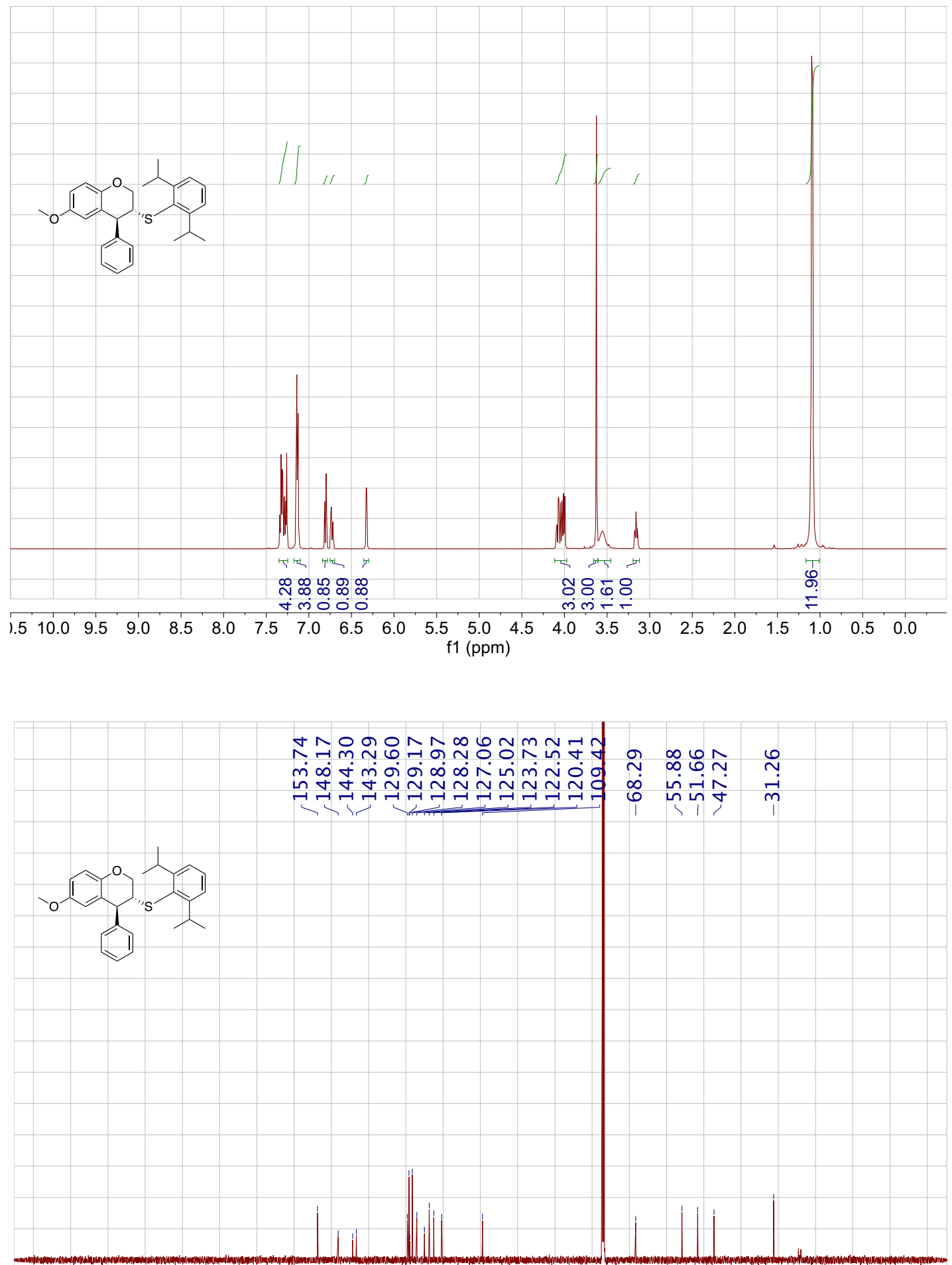

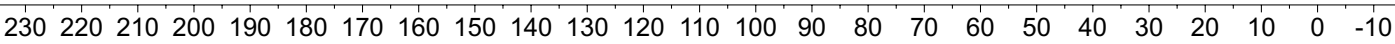
f1 (ppm) 

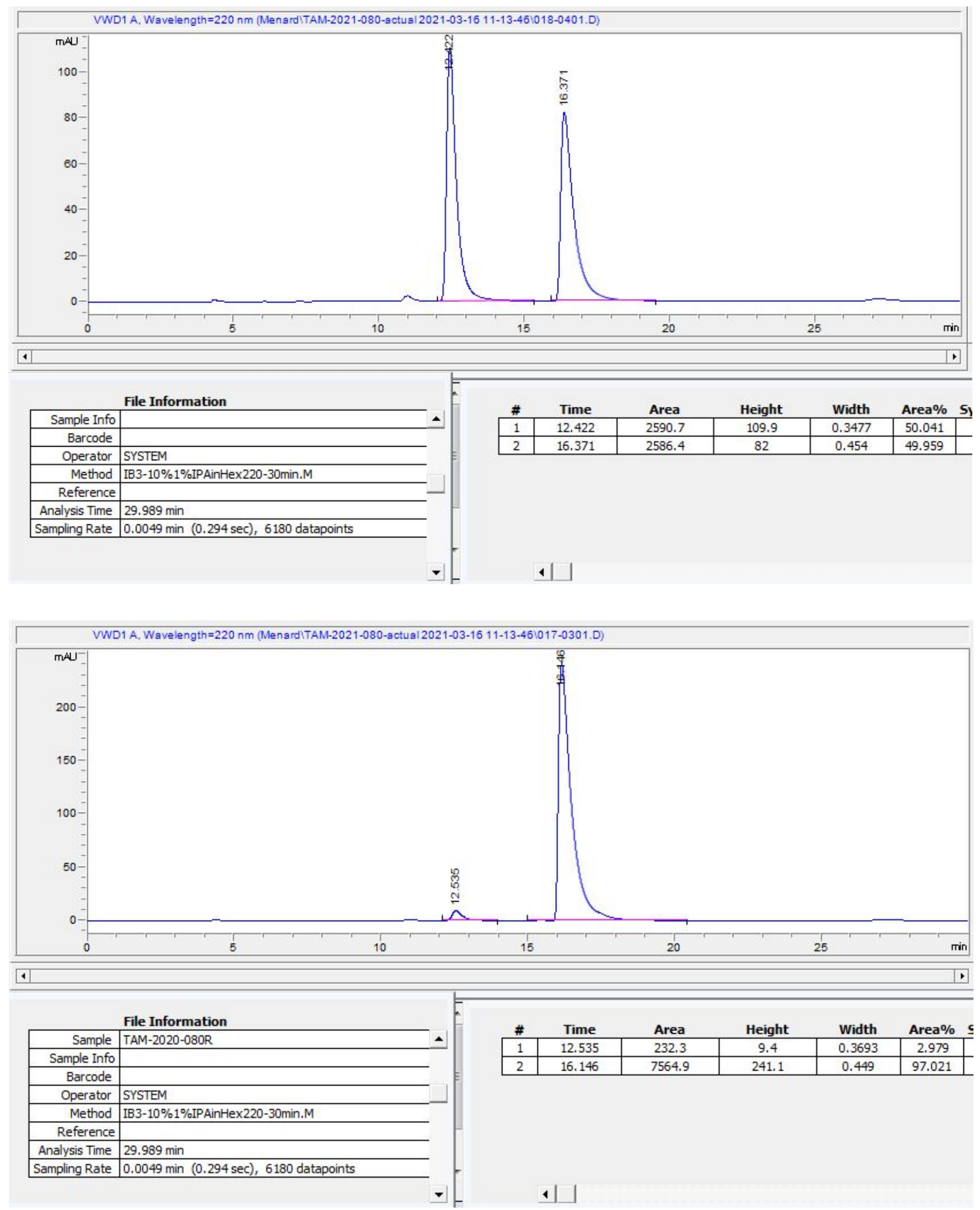
(3R,4S)-3-((2,6-Diisopropylphenyl)thio)-8-methoxy-4-phenylchromane - (3d) - ${ }^{1} \mathrm{H}$ NMR (500 $\left.\mathrm{MHz}, \mathrm{CDCl}_{3}\right) ;{ }^{13} \mathrm{C}\left\{{ }^{1} \mathrm{H}\right\}\left(126 \mathrm{MHz}, \mathrm{CDCl}_{3}\right)$
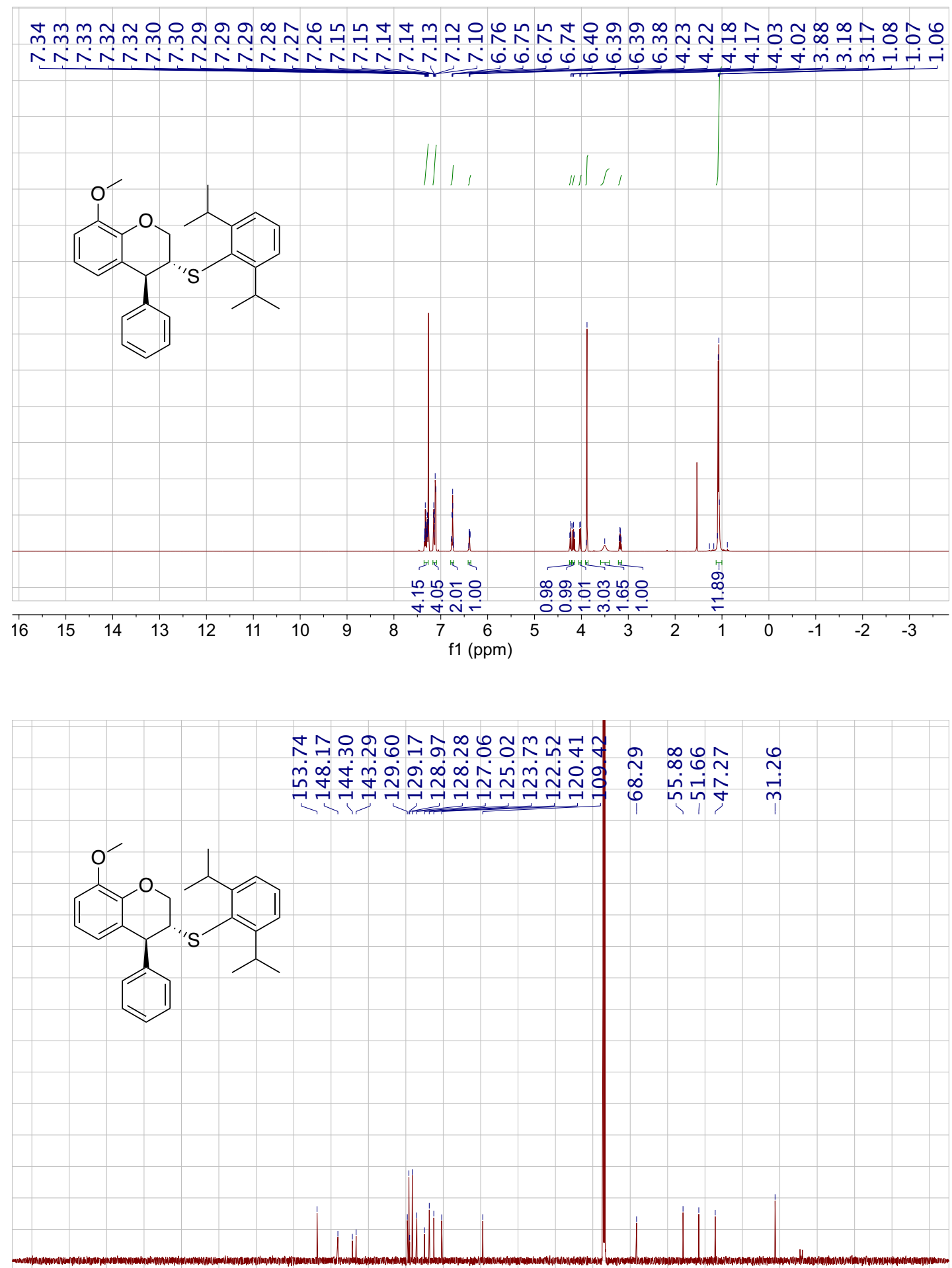

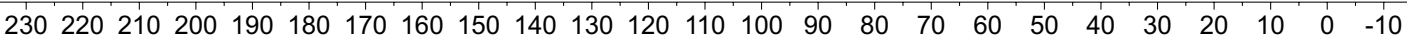
f1 (ppm) 

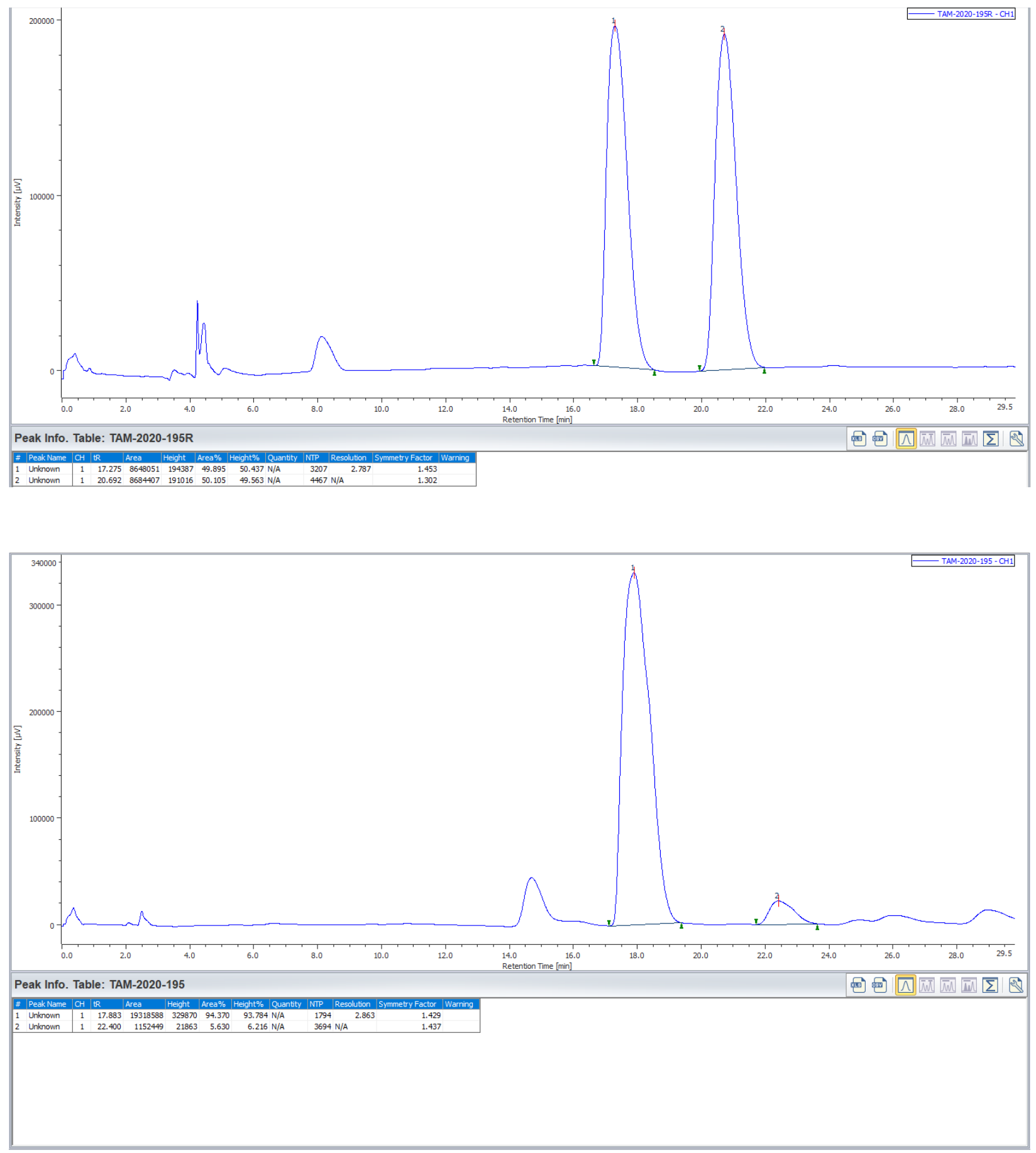
(3S,4R)-6-Bromo-3-((2,6-diisopropylphenyl)thio)-4-phenylchromane - (3e) - ${ }^{1} \mathrm{H}$ NMR (500 $\left.\mathrm{MHz}, \mathrm{CDCl}_{3}\right) ;{ }^{13} \mathrm{C}\left\{{ }^{1} \mathrm{H}\right\}\left(126 \mathrm{MHz}, \mathrm{CDCl}_{3}\right)$
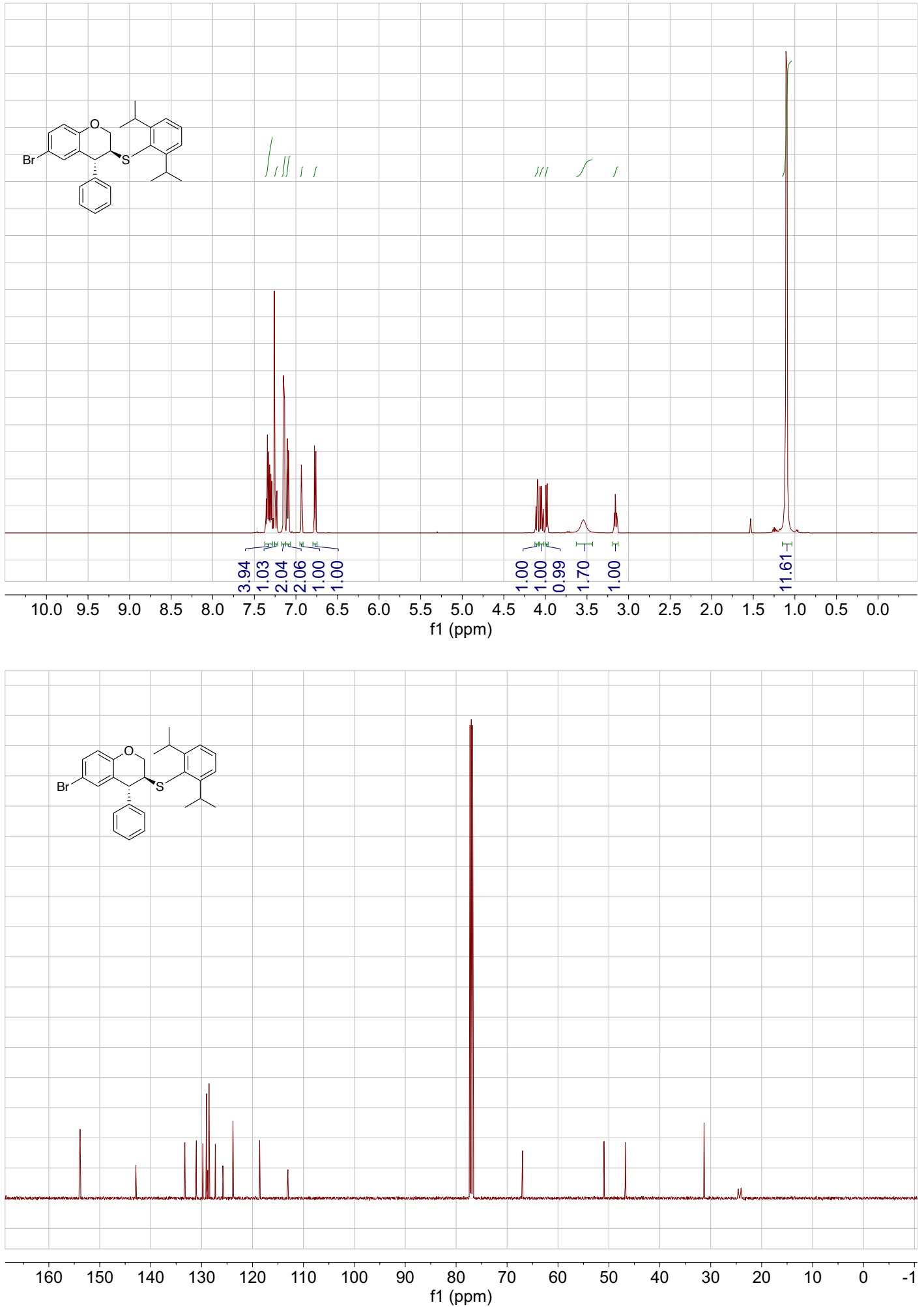

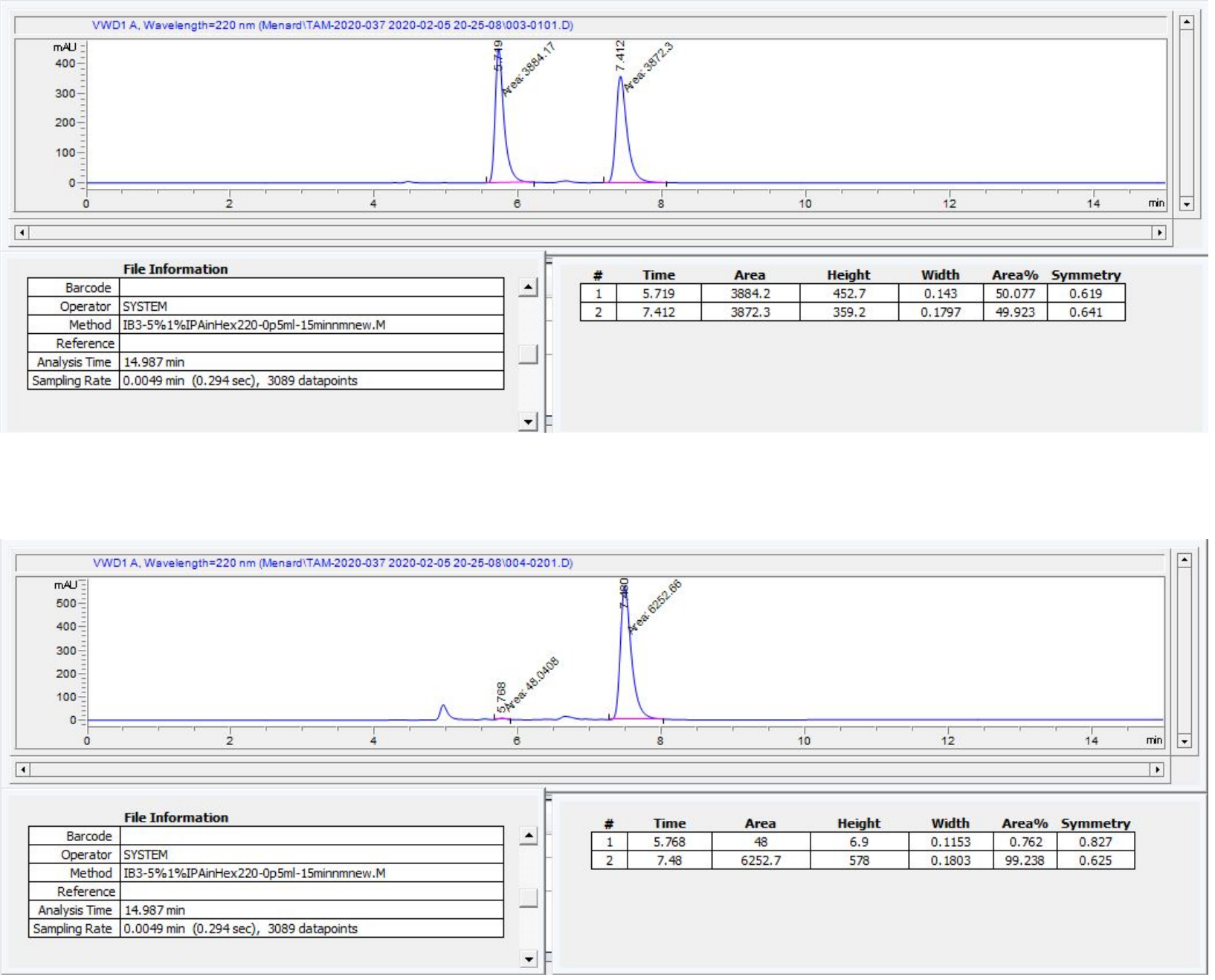
(3S,4R)-3-((2,6-Diisopropylphenyl)thio)-8-fluoro-4-phenylchromane - (3f) - ${ }^{1} \mathrm{H}$ NMR (500 $\left.\mathrm{MHz}, \mathrm{CDCl}_{3}\right) ;{ }^{13} \mathrm{C}\left\{{ }^{1} \mathrm{H}\right\}\left(126 \mathrm{MHz}, \mathrm{CDCl}_{3}\right)$
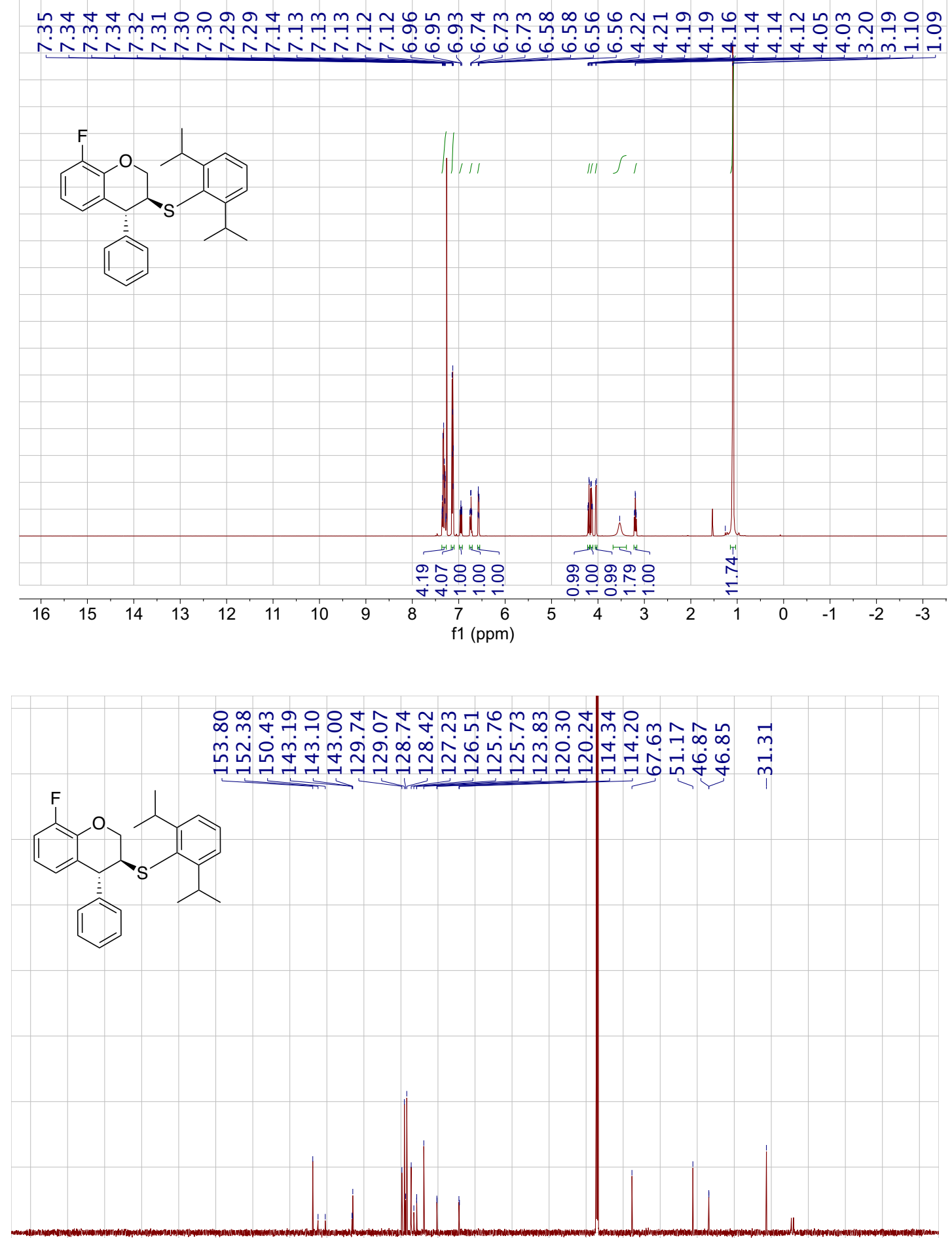

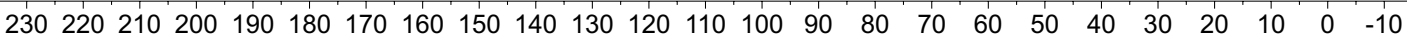



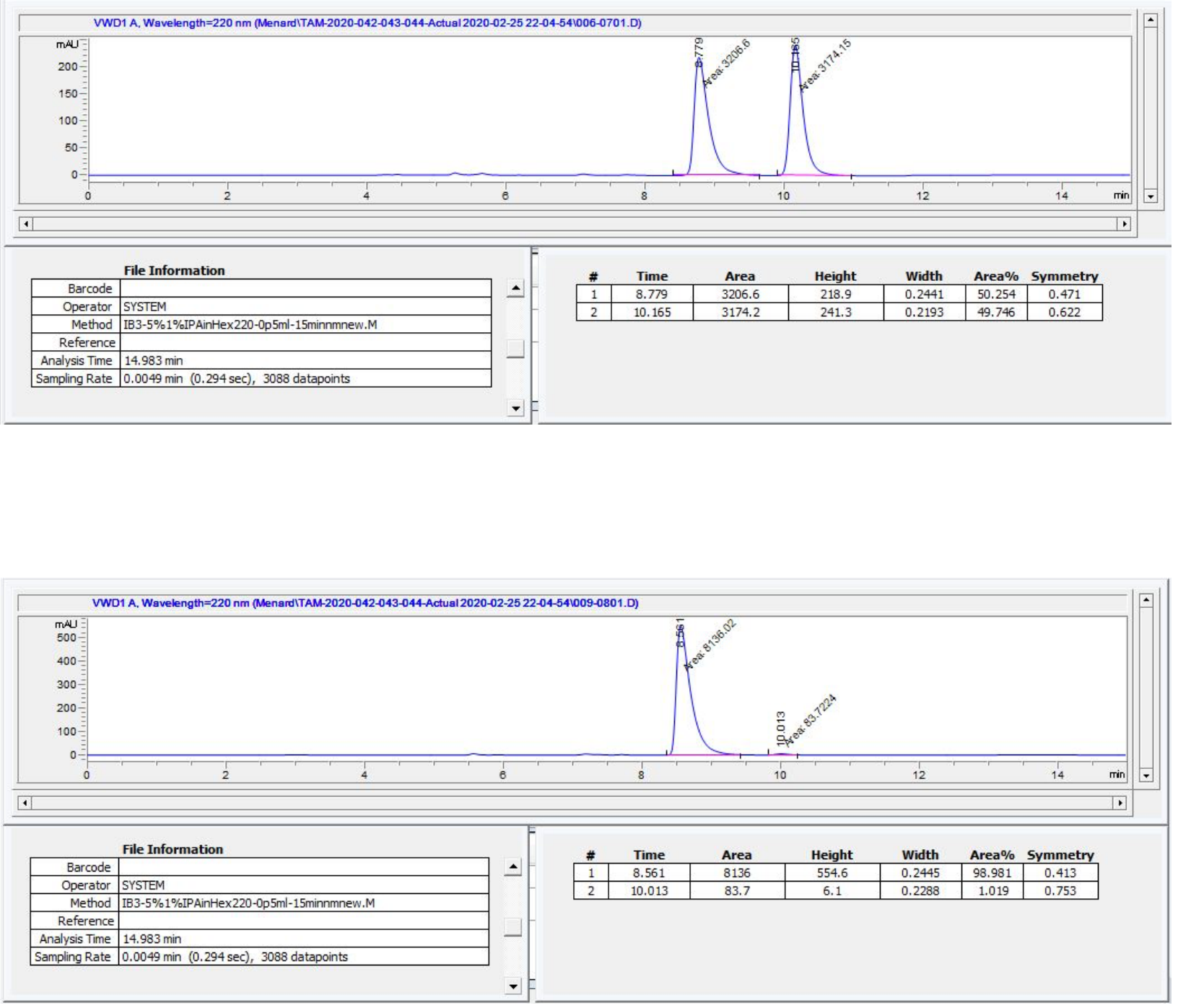
(3S,4R)-3-((2,6-Diisopropylphenyl)thio)-4-phenyl-6-(trifluoromethyl)chromane $-(\mathbf{3 g})-{ }^{1} \mathrm{H}$ $\operatorname{NMR}\left(500 \mathrm{MHz}, \mathrm{CDCl}_{3}\right) ;{ }^{13} \mathrm{C}\left\{{ }^{1} \mathrm{H}\right\}\left(126 \mathrm{MHz}, \mathrm{CDCl}_{3}\right)$
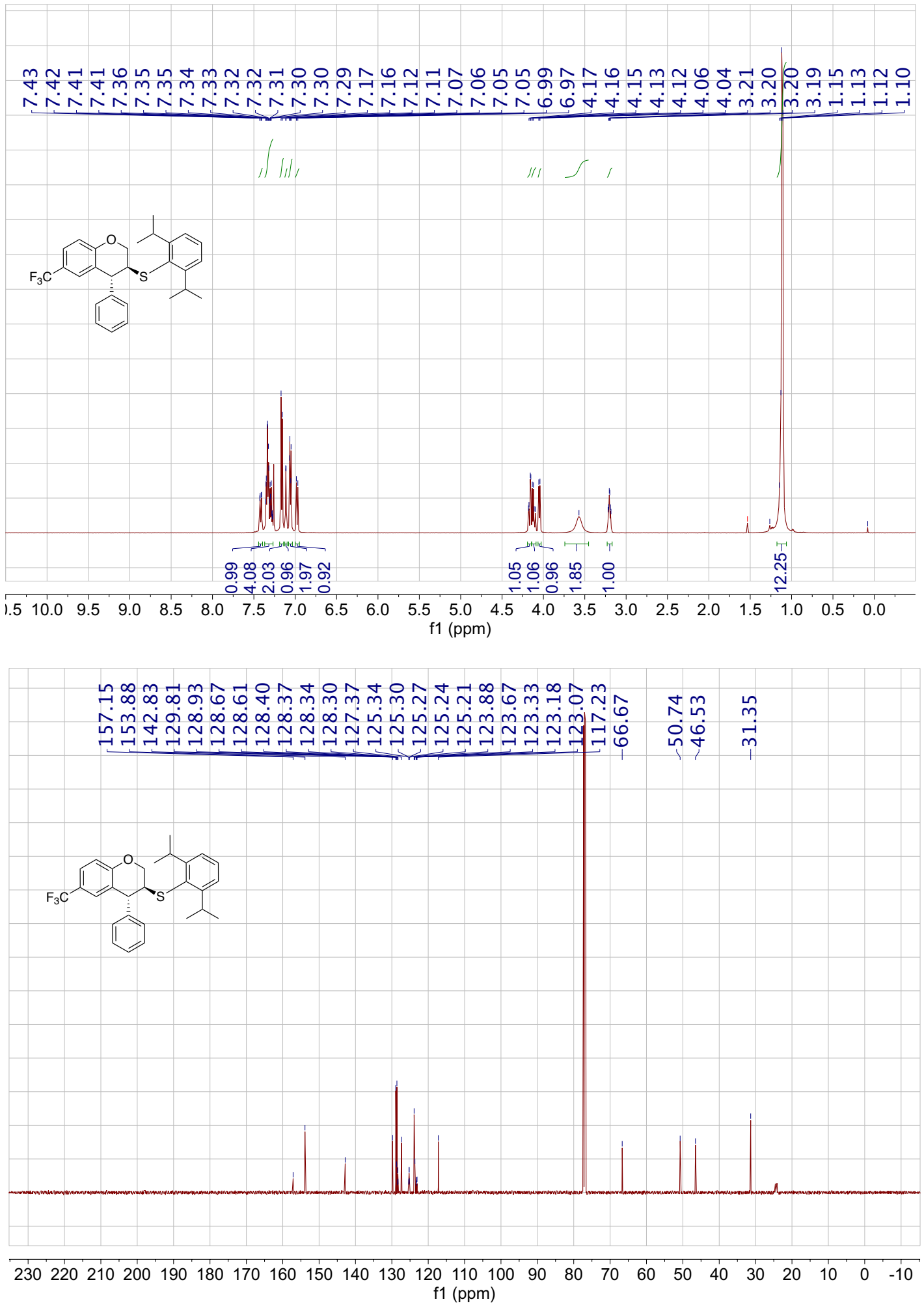

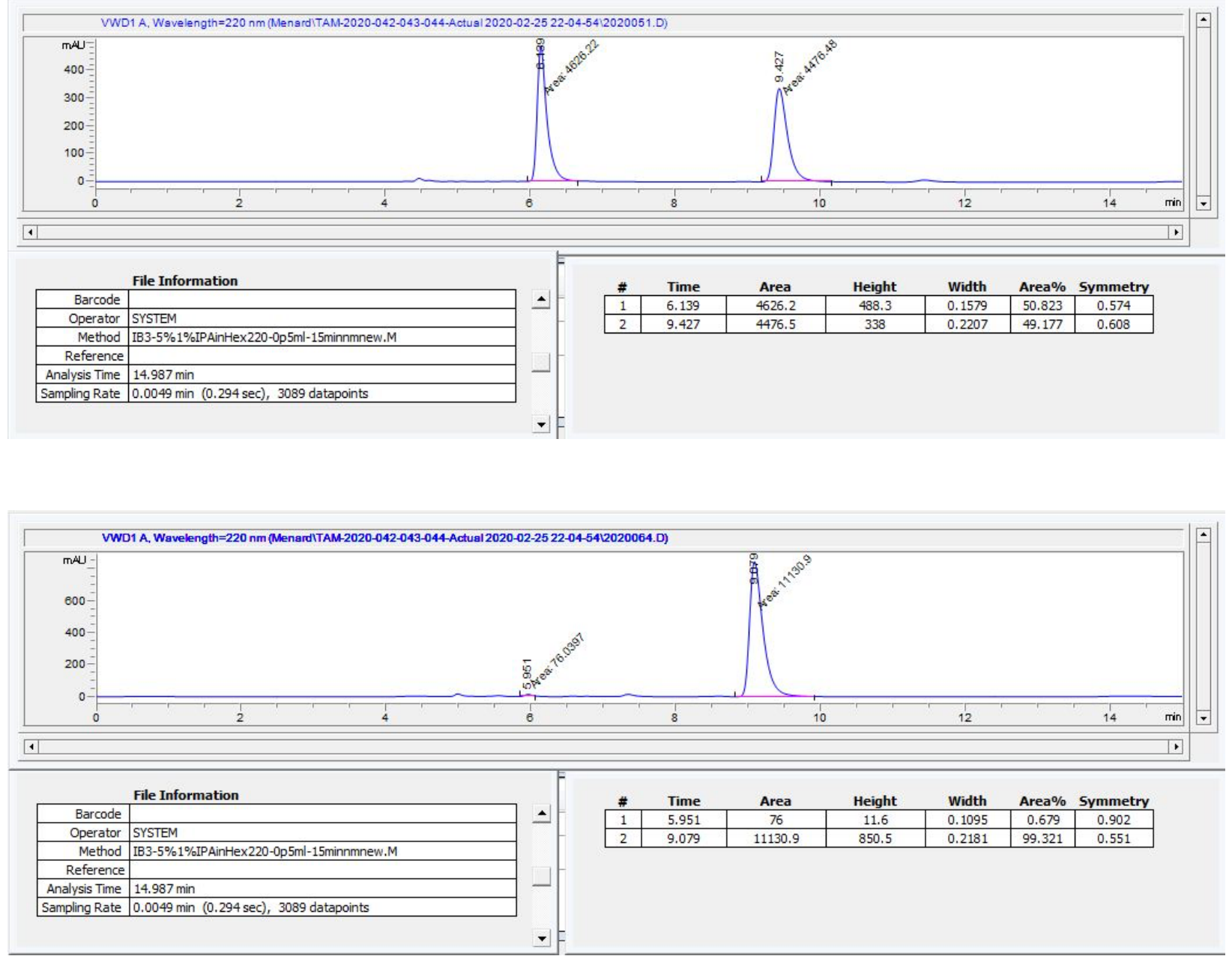
Ethyl (3R,4S)-3-((2,6-diisopropylphenyl)thio)-4-phenylchromane-6-carboxylate $-(\mathbf{3 h})-{ }^{1} \mathrm{H}$ $\operatorname{NMR}\left(500 \mathrm{MHz}, \mathrm{CDCl}_{3}\right) ;{ }^{13} \mathrm{C}\left\{{ }^{1} \mathrm{H}\right\}\left(126 \mathrm{MHz}, \mathrm{CDCl}_{3}\right)$
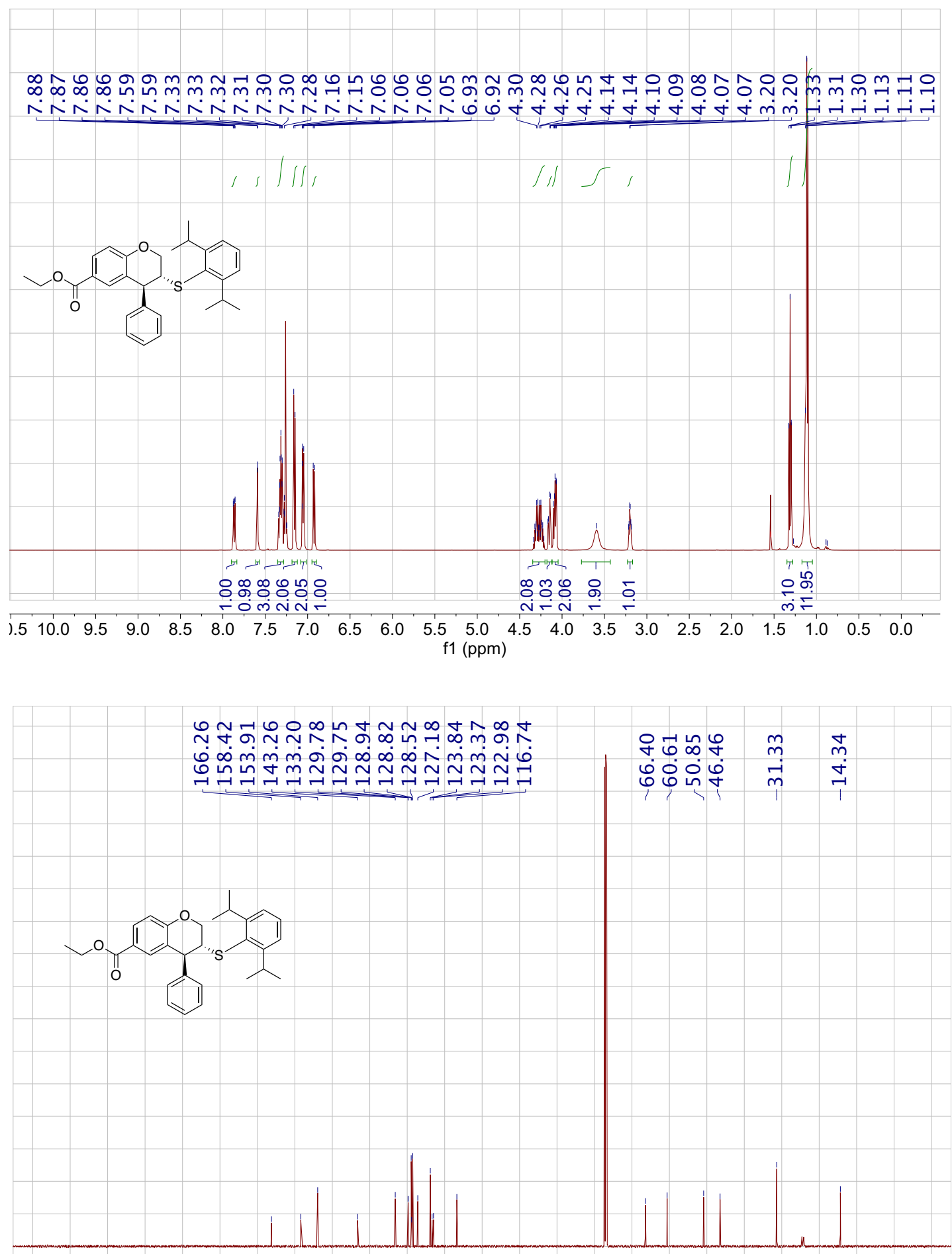

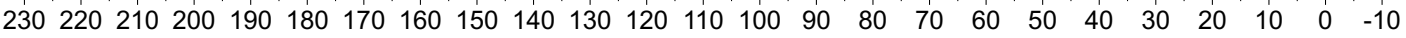
f1 (ppm) 


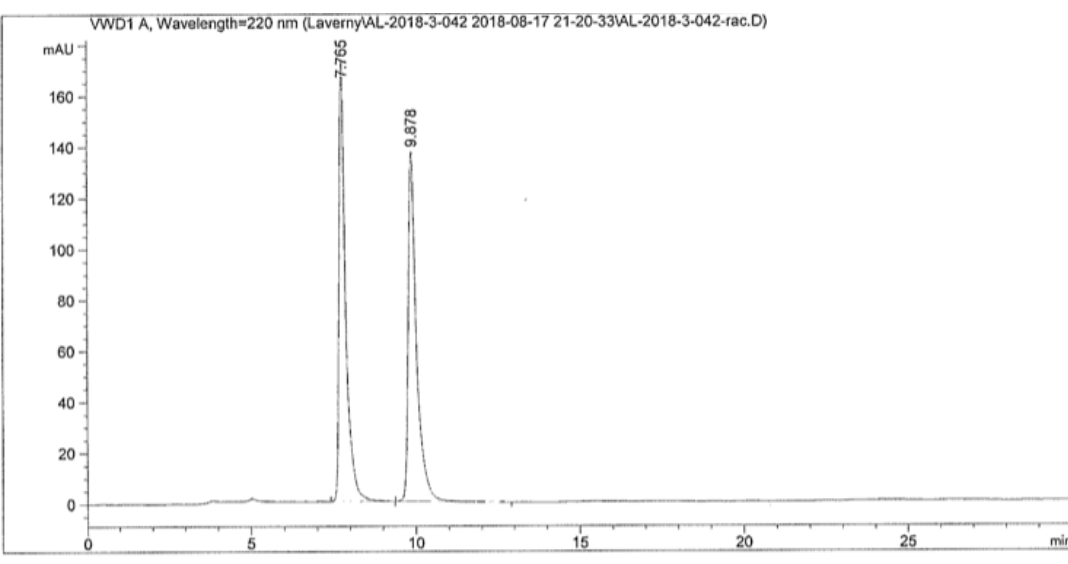

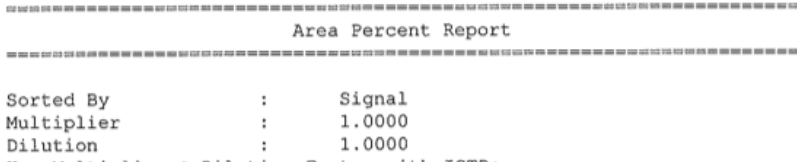

Use Multiplier \& Dilution Factor with ISTDs

Signal 1: VWD1 A, Wavelength $=220 \mathrm{~nm}$

\begin{tabular}{|c|c|c|c|c|c|c|}
\hline eak & $\begin{array}{l}\text { RetTime } \\
\text { [min] }\end{array}$ & Type & $\begin{array}{l}\text { Width } \\
\text { [min] }\end{array}$ & $\begin{array}{c}\text { Area } \\
{[\mathrm{mAU} * \mathrm{~s}]}\end{array}$ & $\begin{array}{l}\text { Height } \\
\text { [MAU] }\end{array}$ & $\begin{array}{c}\text { Area } \\
8\end{array}$ \\
\hline 1 & & 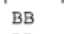 & 0.2 & 2379.7 & 763 & 039 \\
\hline 2 & 9.878 & BB & 0.2511 & 2369.86108 & 137.12048 & 49.8961 \\
\hline
\end{tabular}

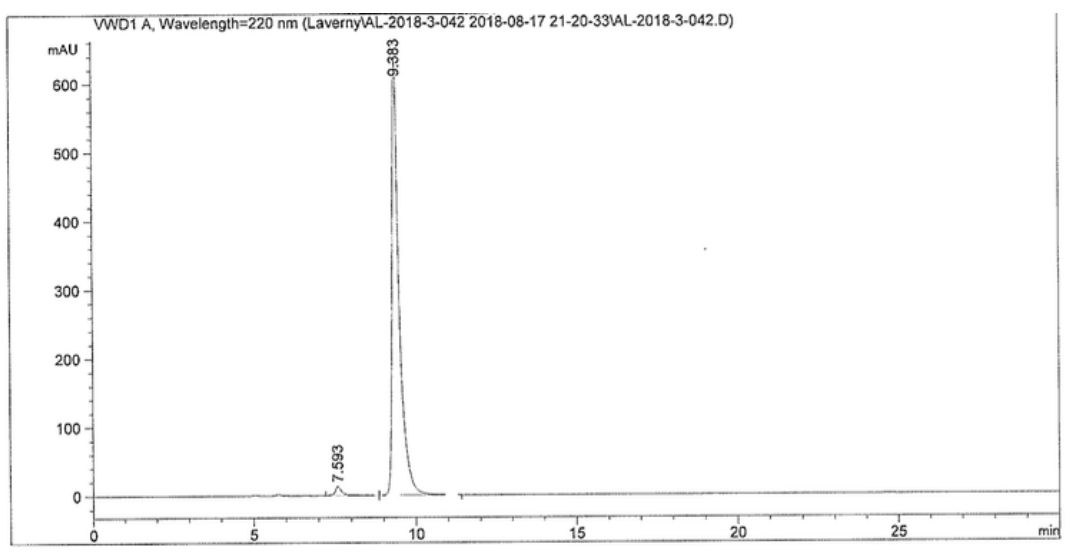

A.rea Percent Report

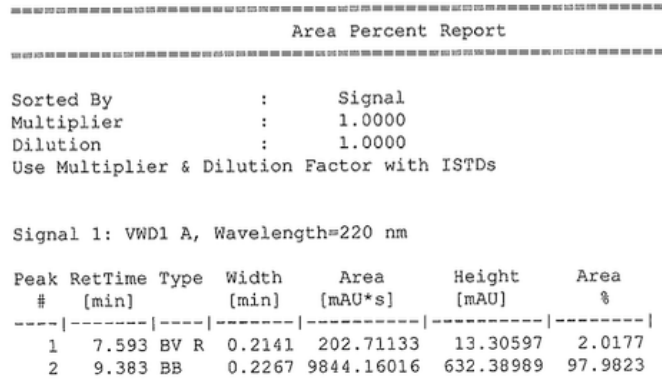


$N$-((3R,4S)-3-((2,6-Diisopropylphenyl)thio)-4-phenylchroman-6-yl)acetamide - (3i) - ${ }^{1} \mathrm{H}$ NMR $\left(500 \mathrm{MHz}, \mathrm{CDCl}_{3}\right) ;{ }^{13} \mathrm{C}\left\{{ }^{1} \mathrm{H}\right\}\left(126 \mathrm{MHz}, \mathrm{CDCl}_{3}\right)$
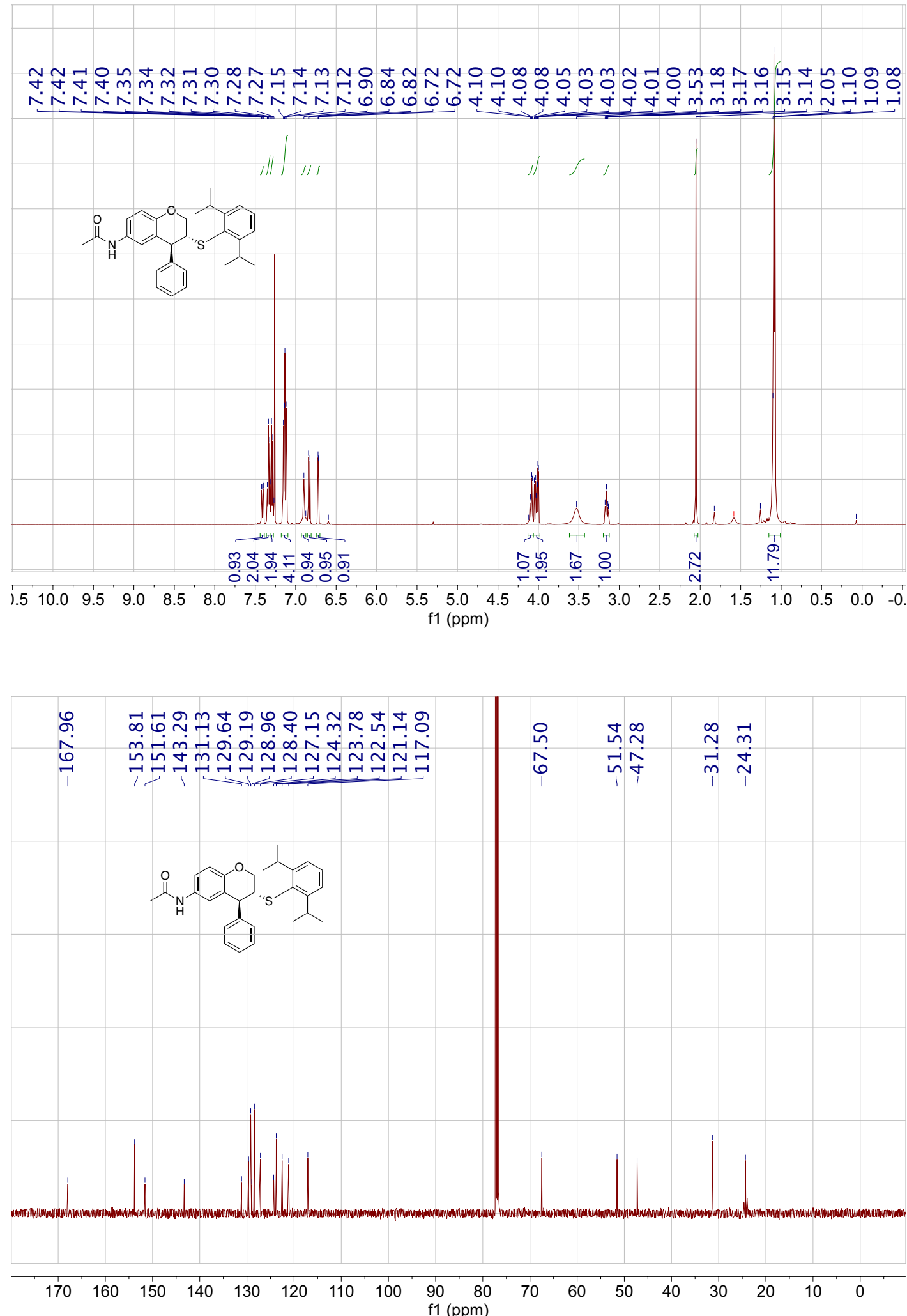

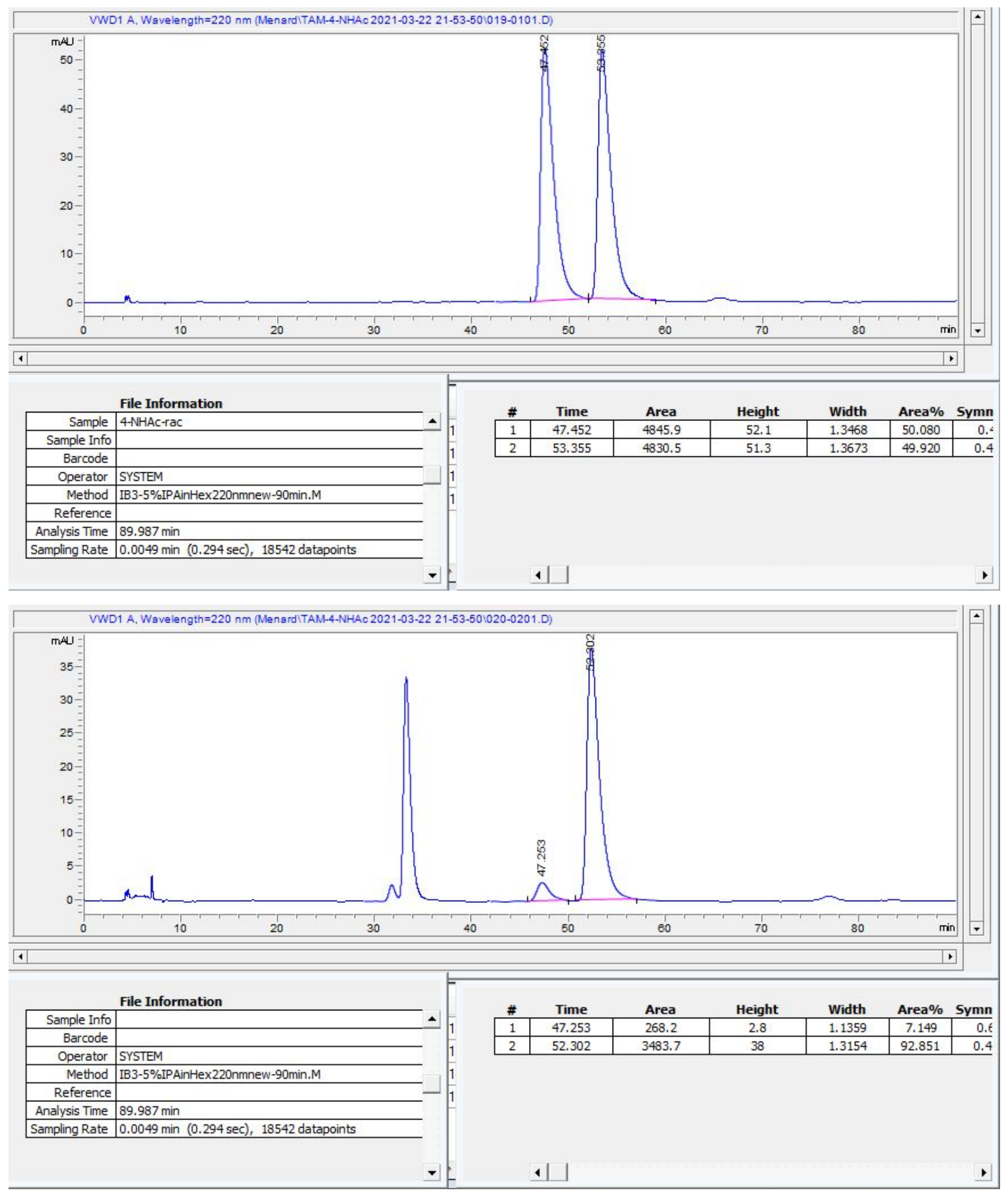
$(3 R, 4 S)-3-((2,6-D i i s o p r o p y l p h e n y l) t h i o)-7-m e t h o x y-4-p h e n y l c h r o m a n e-(3 \mathbf{j})-{ }^{1} \mathrm{H}$ NMR (500 $\left.\mathrm{MHz}, \mathrm{CDCl}_{3}\right) ;{ }^{13} \mathrm{C}\left\{{ }^{1} \mathrm{H}\right\}\left(126 \mathrm{MHz}, \mathrm{CDCl}_{3}\right)$
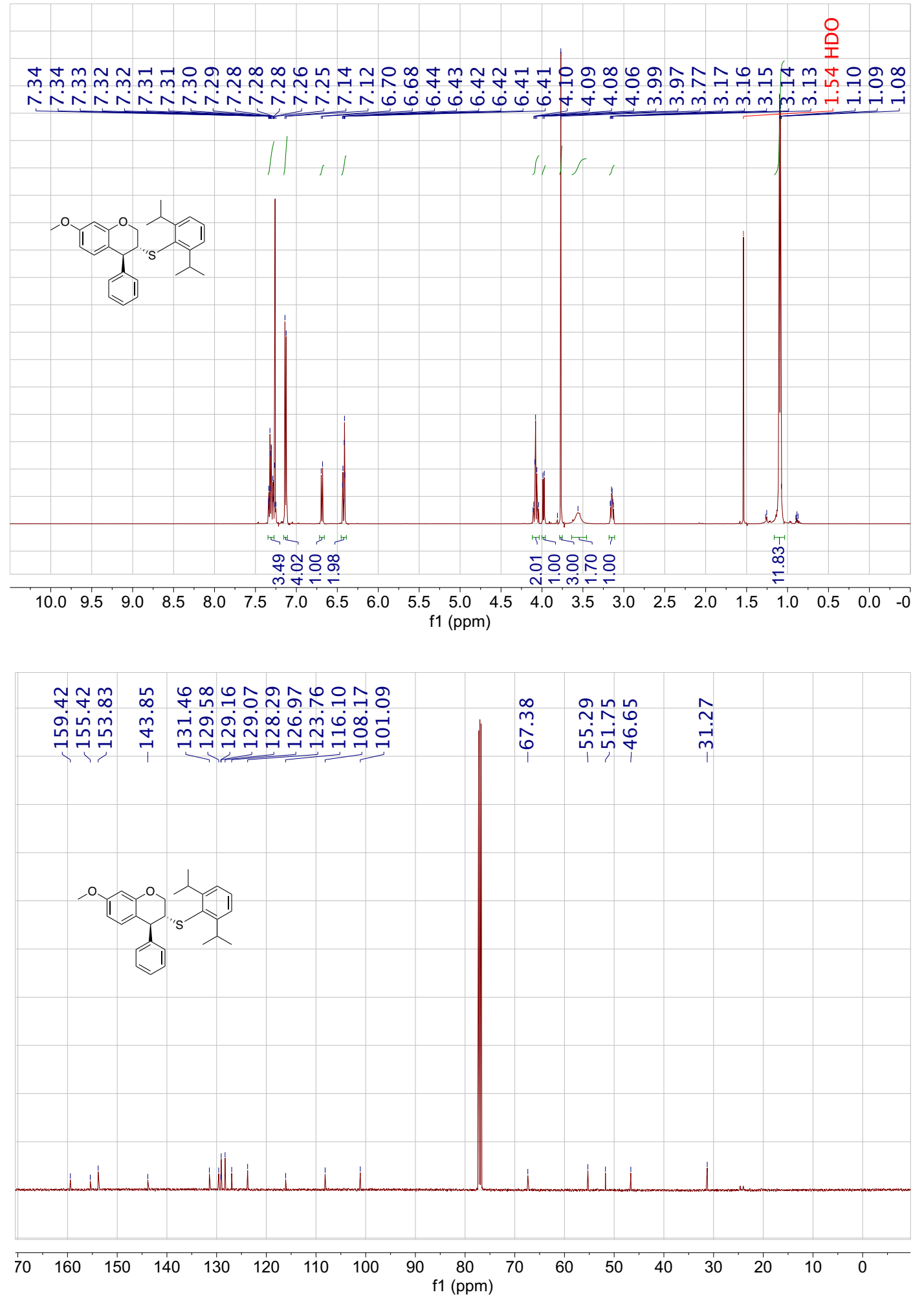


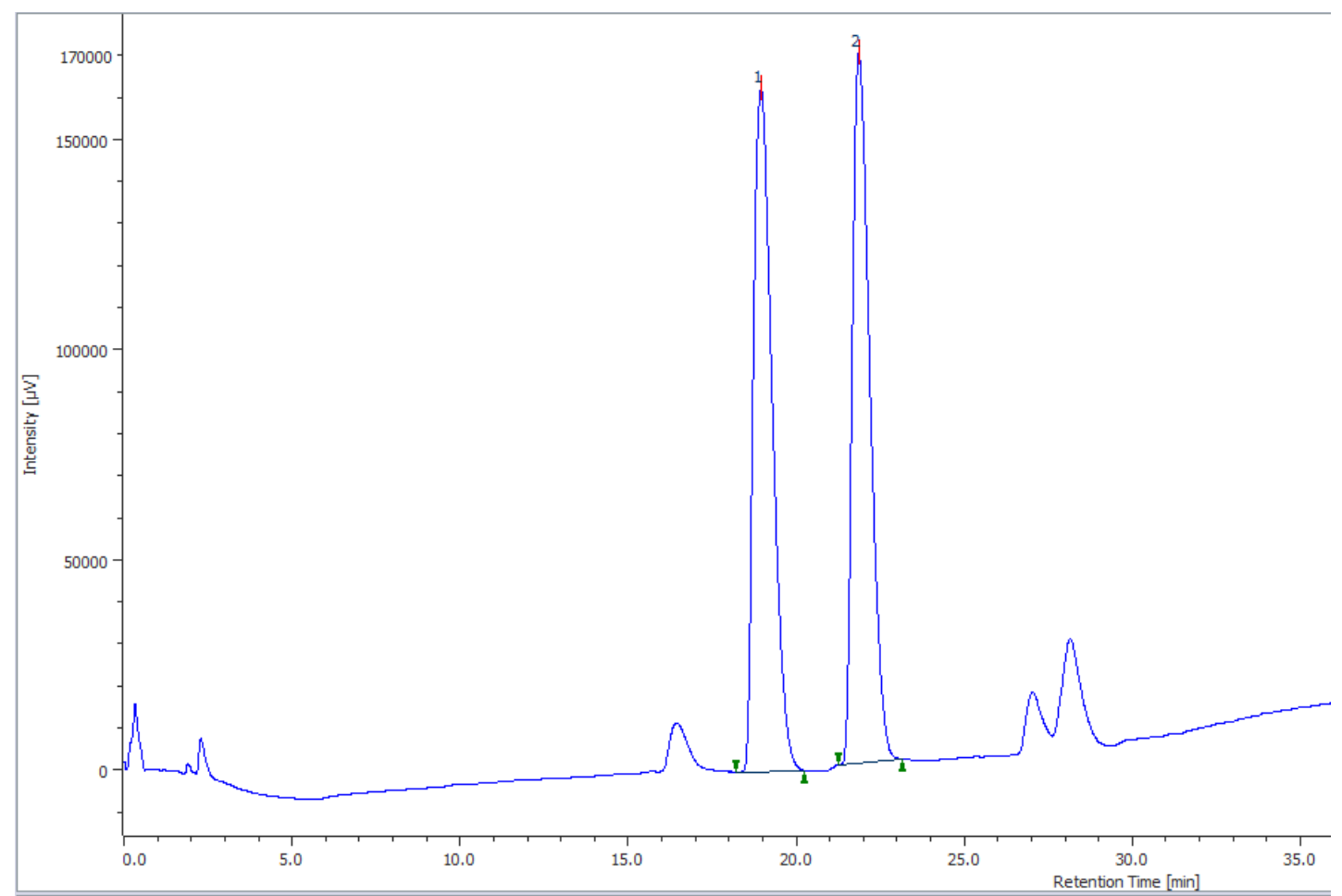

Peak Info. Table: TAM-2020-188R

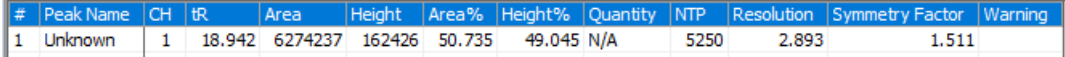

\begin{tabular}{|ll|lllllllll|}
1 & Unknown & 1 & 18.942 & 6274237 & 162426 & 50.735 & $49.045 \mathrm{~N} / \mathrm{A}$ & 5250 & 2.893 & 1.511 \\
2 & Unknown & 1 & 21.850 & 6092377 & 168749 & 49.265 & $50.955 \mathrm{~N} / \mathrm{A}$ & $8109 \mathrm{~N} / \mathrm{A}$ & & 1.555 \\
\hline
\end{tabular}

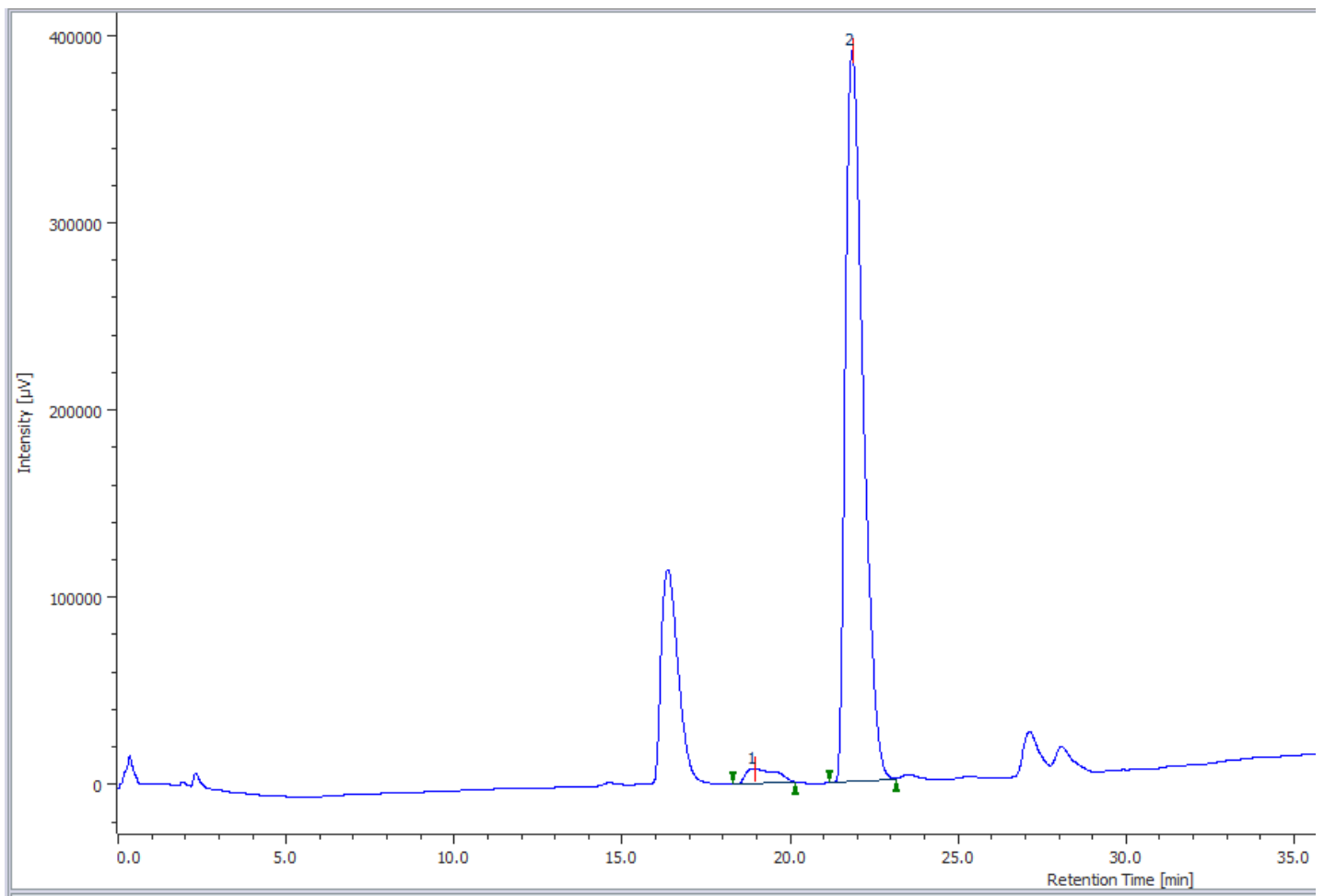

Peak Info. Table: TAM-2020-184

\begin{tabular}{||l|r|r|r|r|r|r|r|r|r|r|r||}
\hline \# & Peak Name & CH & tR & Area & Height & Area \% & Height \% & Quantity & NTP & Resolution & Symmetry Factor Warning \\
\hline 1 & Unknown & 1 & 18.942 & 465666 & 7731 & 3.144 & 1.946 N/A & 1569 & 2.012 & 1.796 \\
2 & Unknown & 1 & 21.842 & 14343955 & 389614 & 96.856 & 98.054 N/A & 7993 & N/A & 1.536 \\
\hline
\end{tabular}


(3S,4R)-3-((2,6-Diisopropylphenyl)thio)-5-nitro-4-phenylchromane - (3k ) - ${ }^{1} \mathrm{H}$ NMR (500 $\left.\mathrm{MHz}, \mathrm{CDCl}_{3}\right) ;{ }^{13} \mathrm{C}\left\{{ }^{1} \mathrm{H}\right\}\left(126 \mathrm{MHz}, \mathrm{CDCl}_{3}\right)$
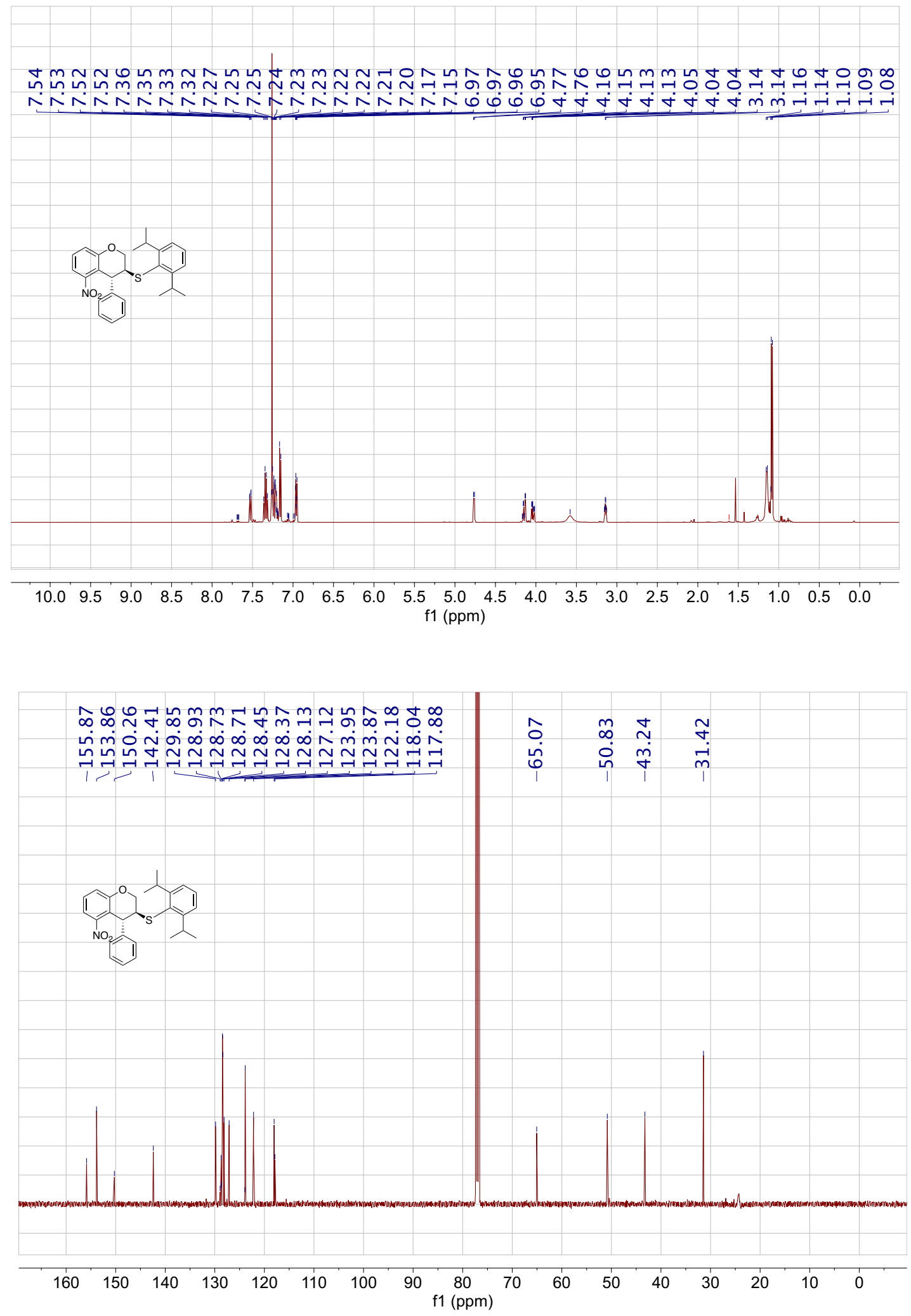

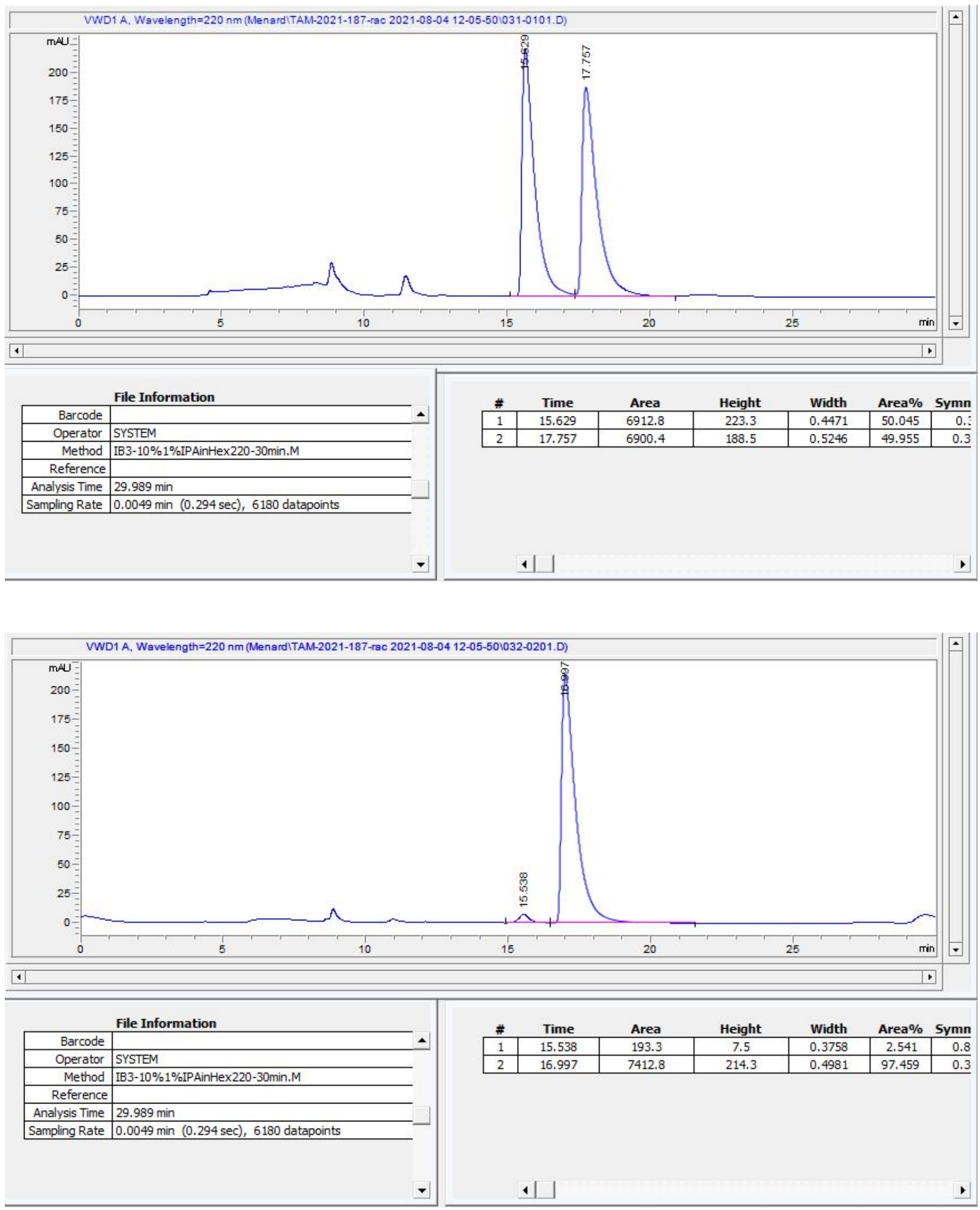
(3R,4R)-4-(2-Bromophenyl)-3-((2,6-diisopropylphenyl)thio)chromane - (4a) - ${ }^{1} \mathrm{H}$ NMR (500 $\left.\mathrm{MHz}, \mathrm{CDCl}_{3}\right) ;{ }^{13} \mathrm{C}\left\{{ }^{1} \mathrm{H}\right\}\left(126 \mathrm{MHz}, \mathrm{CDCl}_{3}\right)$
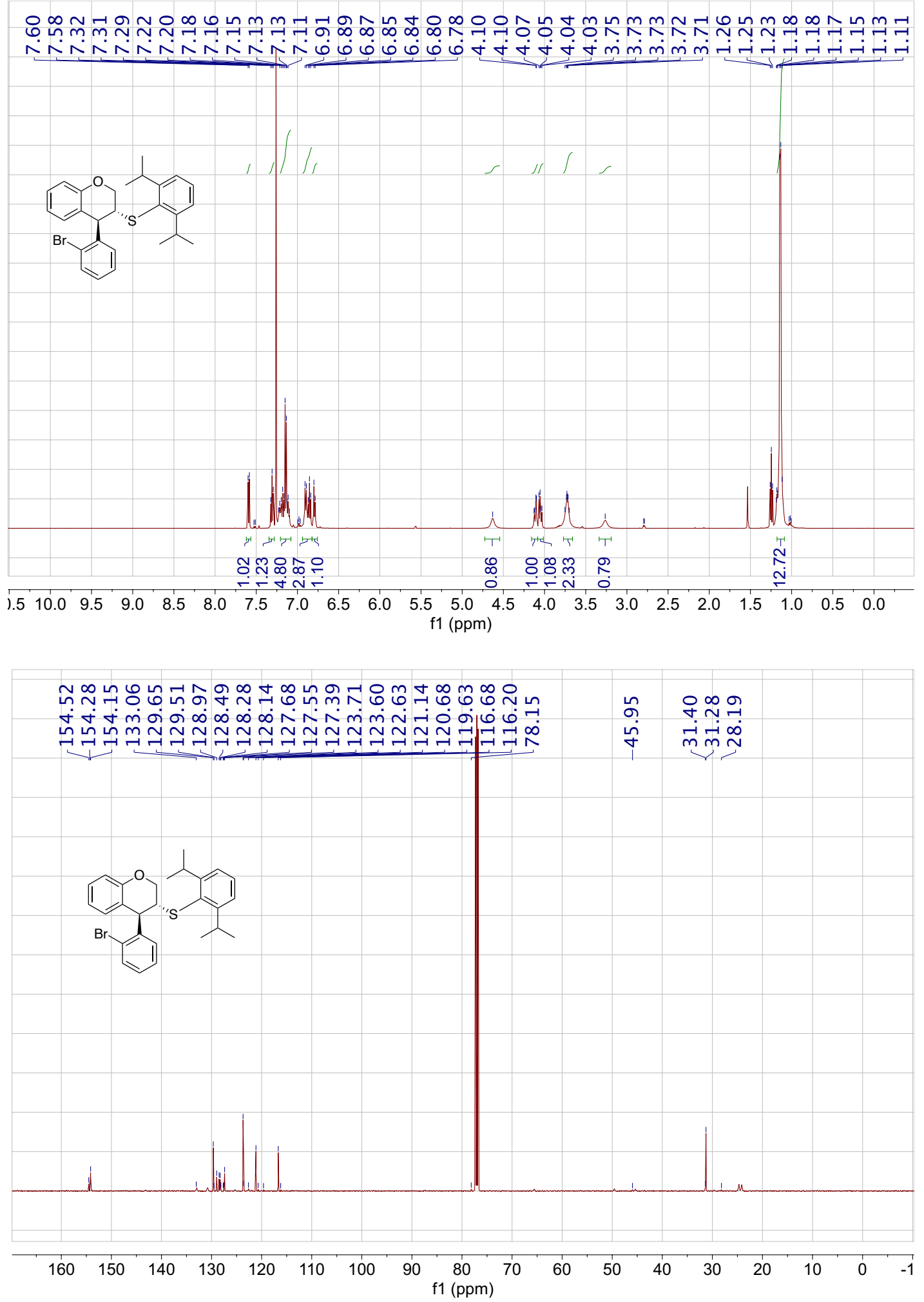

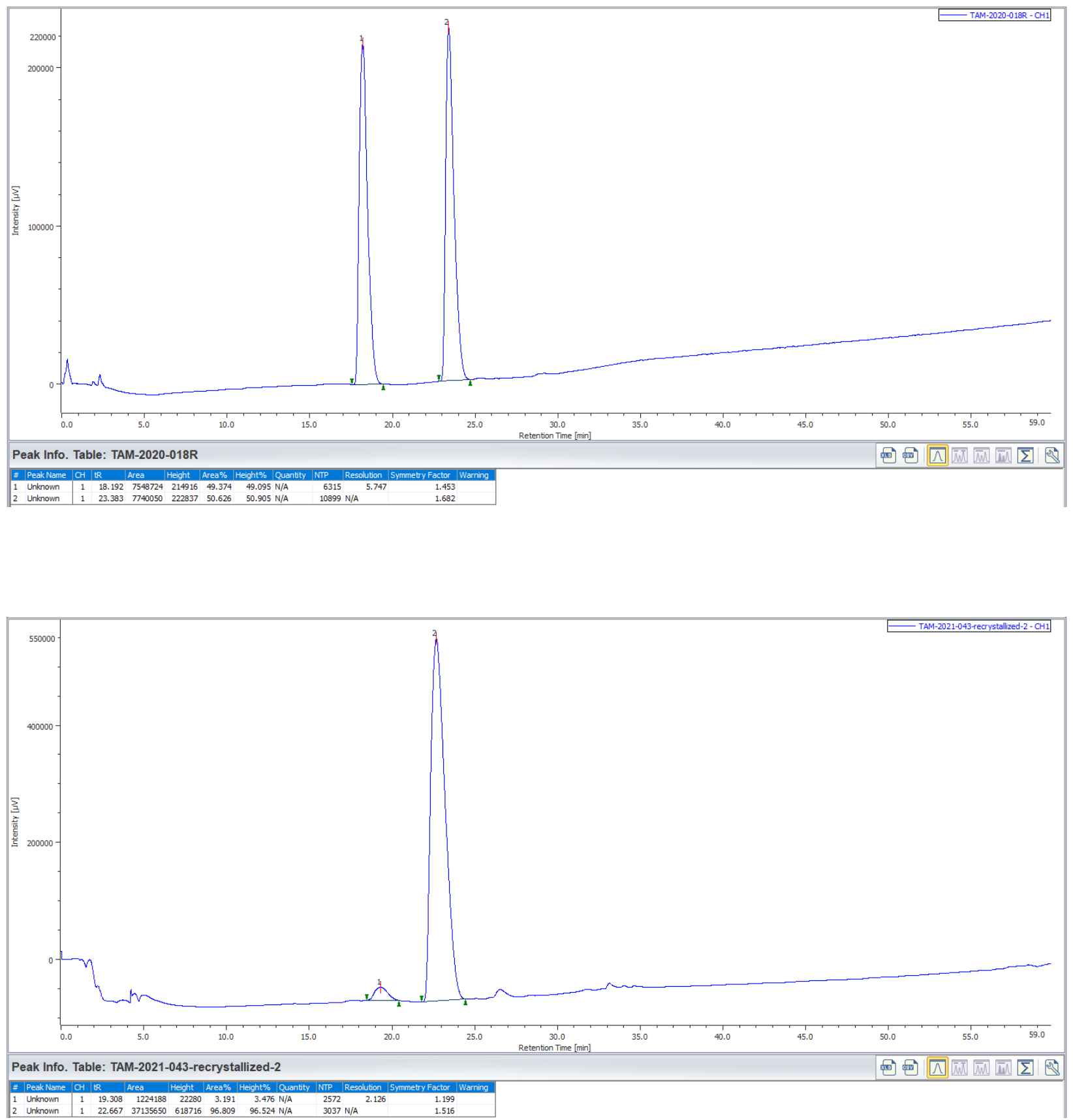
(3R,4S)-4-(4-Chlorophenyl)-3-((2,6-diisopropylphenyl)thio)chromane - (4b) - ${ }^{1} \mathrm{H}$ NMR (500 $\left.\mathrm{MHz}, \mathrm{CDCl}_{3}\right) ;{ }^{13} \mathrm{C}\left\{{ }^{1} \mathrm{H}\right\}\left(126 \mathrm{MHz}, \mathrm{CDCl}_{3}\right)$
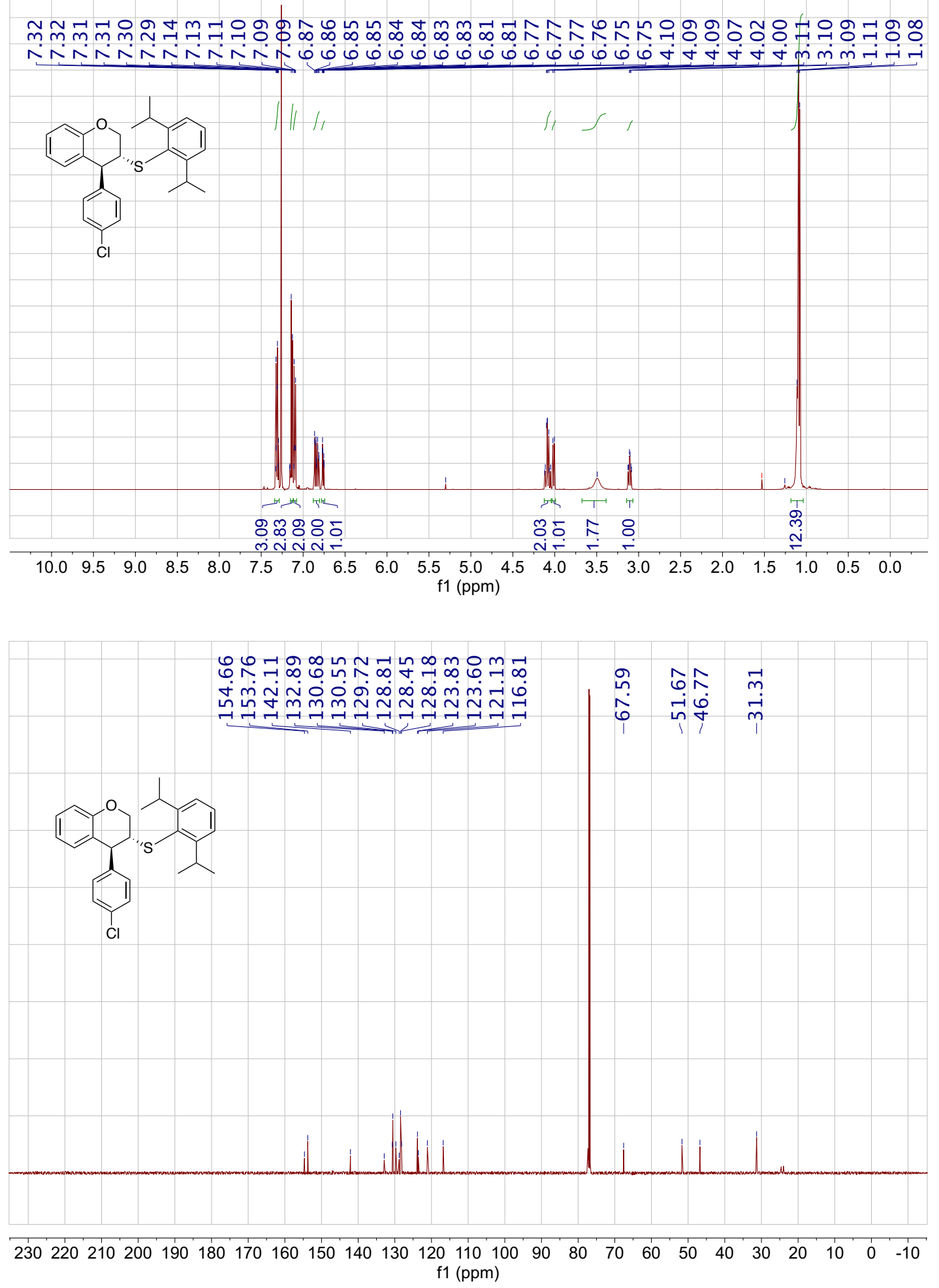

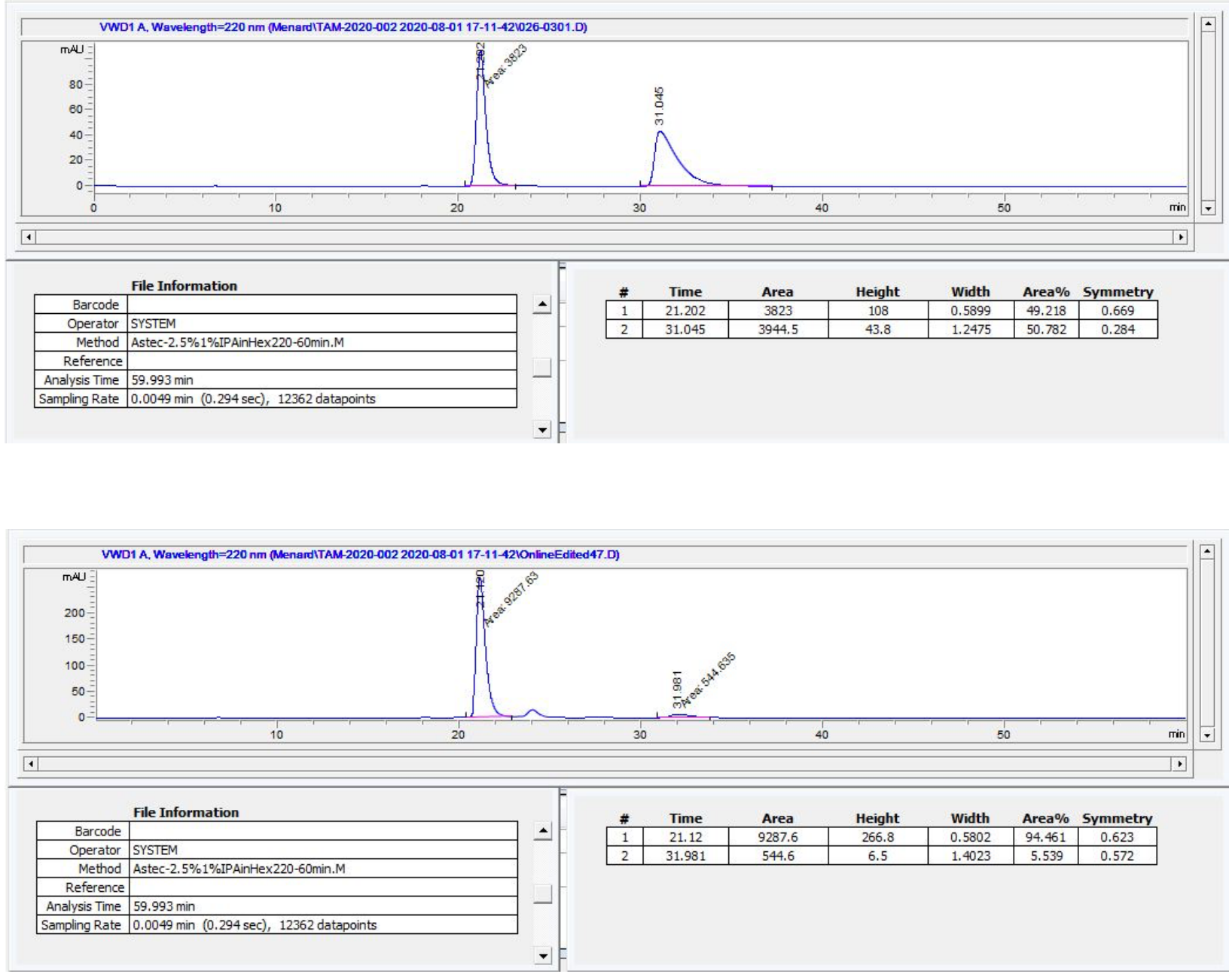
$(3 R, 4 S)-4-\left(4-(B e n z y l o x y)\right.$ phenyl)-3-((2,6-diisopropylphenyl)thio)chromane - (4c) - ${ }^{1} \mathrm{H}$ NMR $\left(500 \mathrm{MHz}, \mathrm{CDCl}_{3}\right) ;{ }^{13} \mathrm{C}\left\{{ }^{1} \mathrm{H}\right\}\left(126 \mathrm{MHz}, \mathrm{CDCl}_{3}\right)$
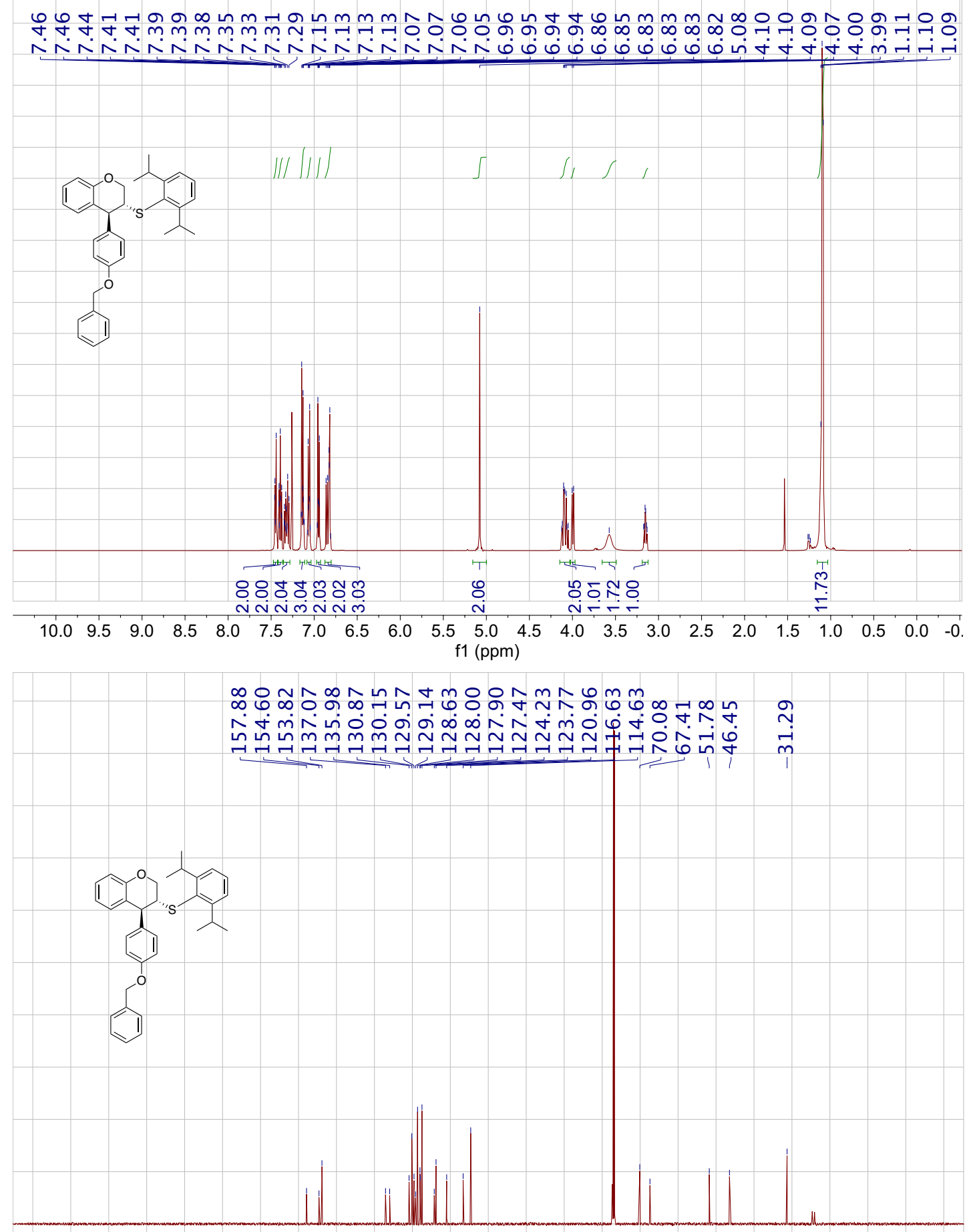

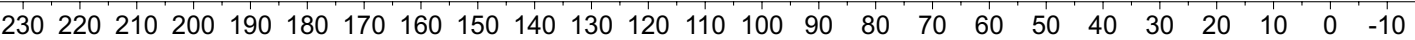
$\mathrm{f1}(\mathrm{ppm})$ 

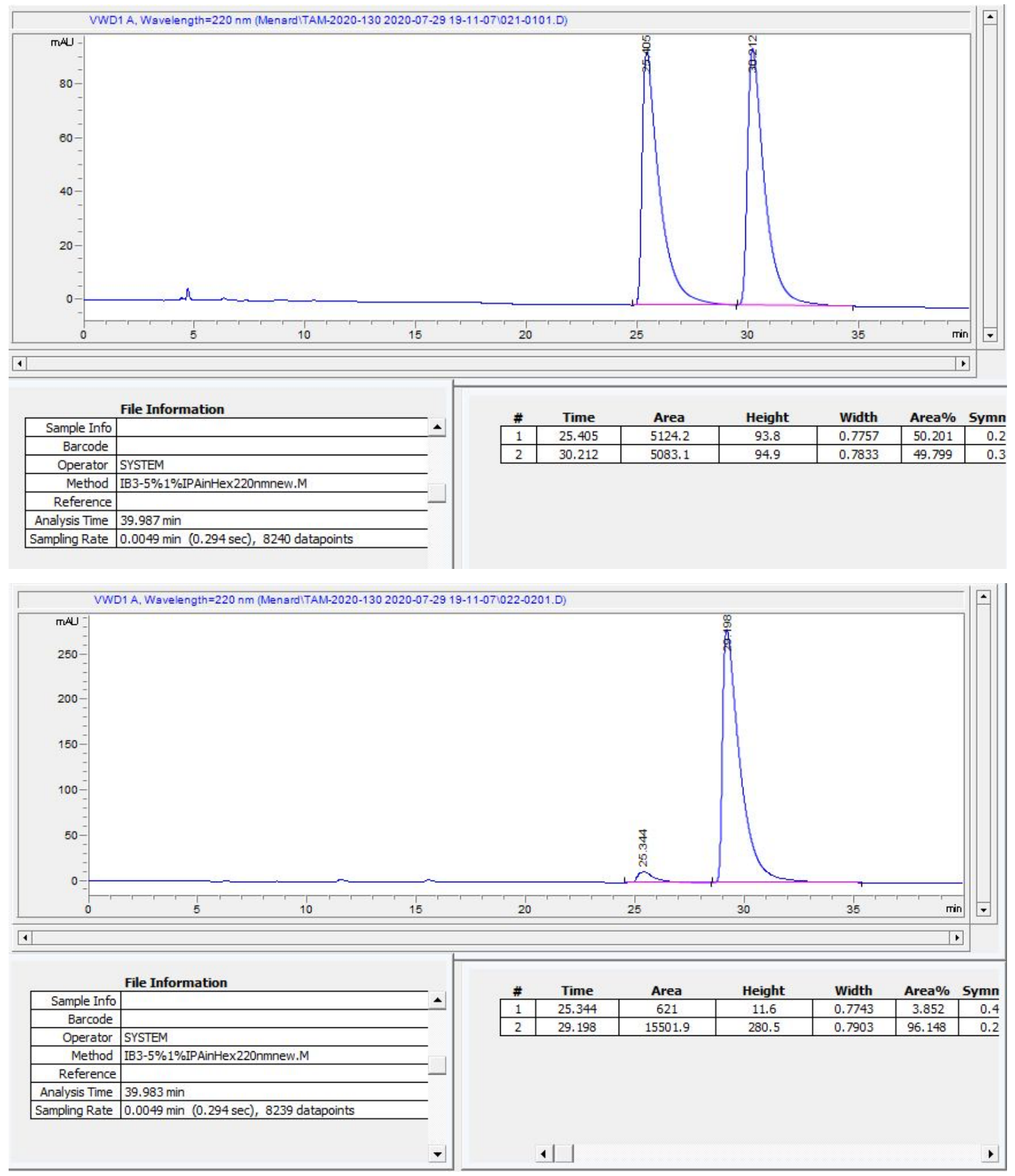
(3S,4R)-3-((2,6-Diisopropylphenyl)sulfinyl)-4-phenylchromane (7) - ${ }^{1} \mathrm{H}$ NMR $(500 \mathrm{MHz}$, $\left.\mathrm{CDCl}_{3}\right) ;{ }^{13} \mathrm{C}\left\{{ }^{1} \mathrm{H}\right\}\left(126 \mathrm{MHz}, \mathrm{CDCl}_{3}\right)$
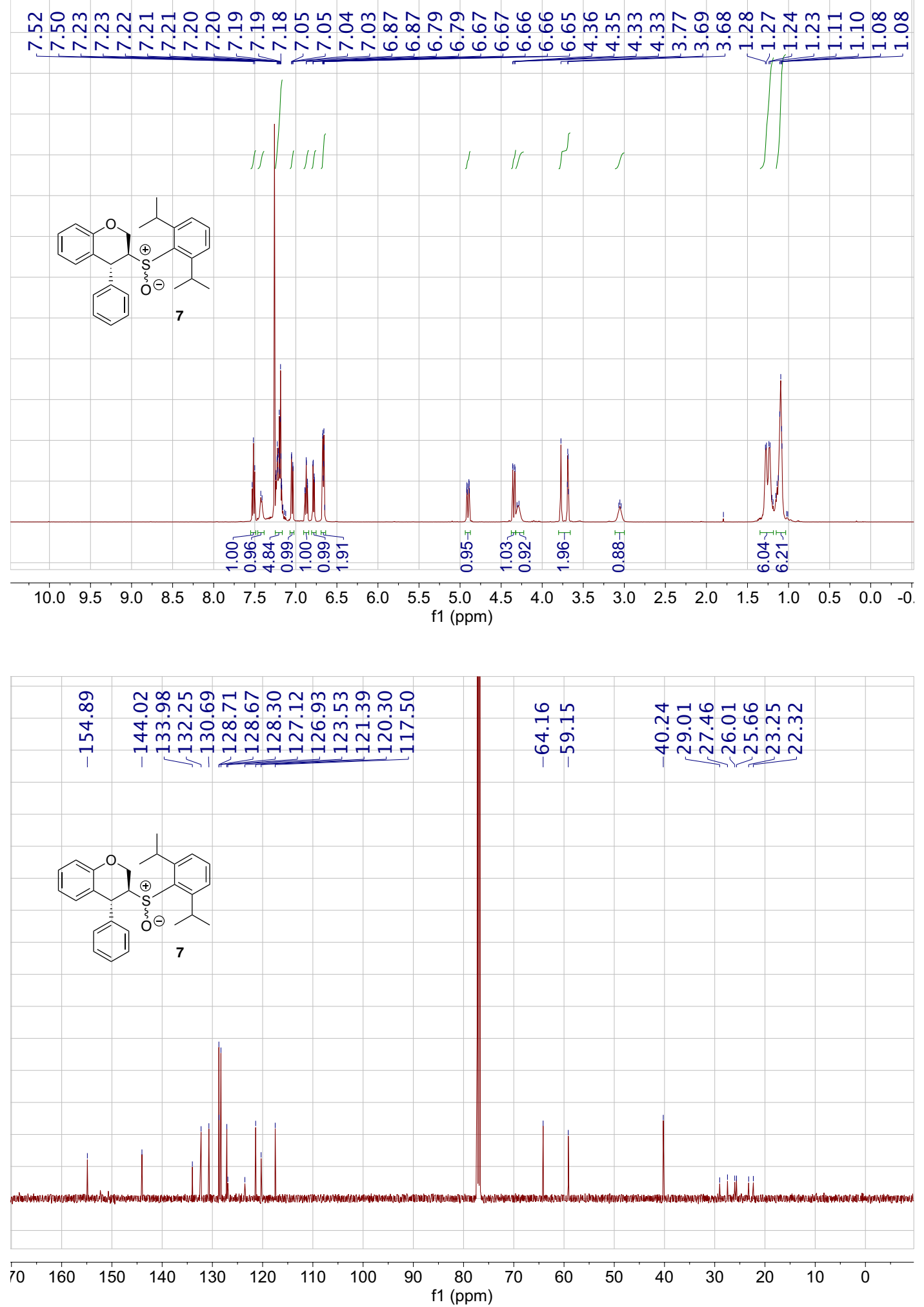
(3S,4R)-3-((2,6-Diisopropylphenyl)sulfonyl)-4-phenylchromane (8) - ${ }^{1} \mathrm{H}$ NMR $(500 \mathrm{MHz}$, $\left.\mathrm{CDCl}_{3}\right) ;{ }^{13} \mathrm{C}\left\{{ }^{1} \mathrm{H}\right\}\left(126 \mathrm{MHz}, \mathrm{CDCl}_{3}\right)$
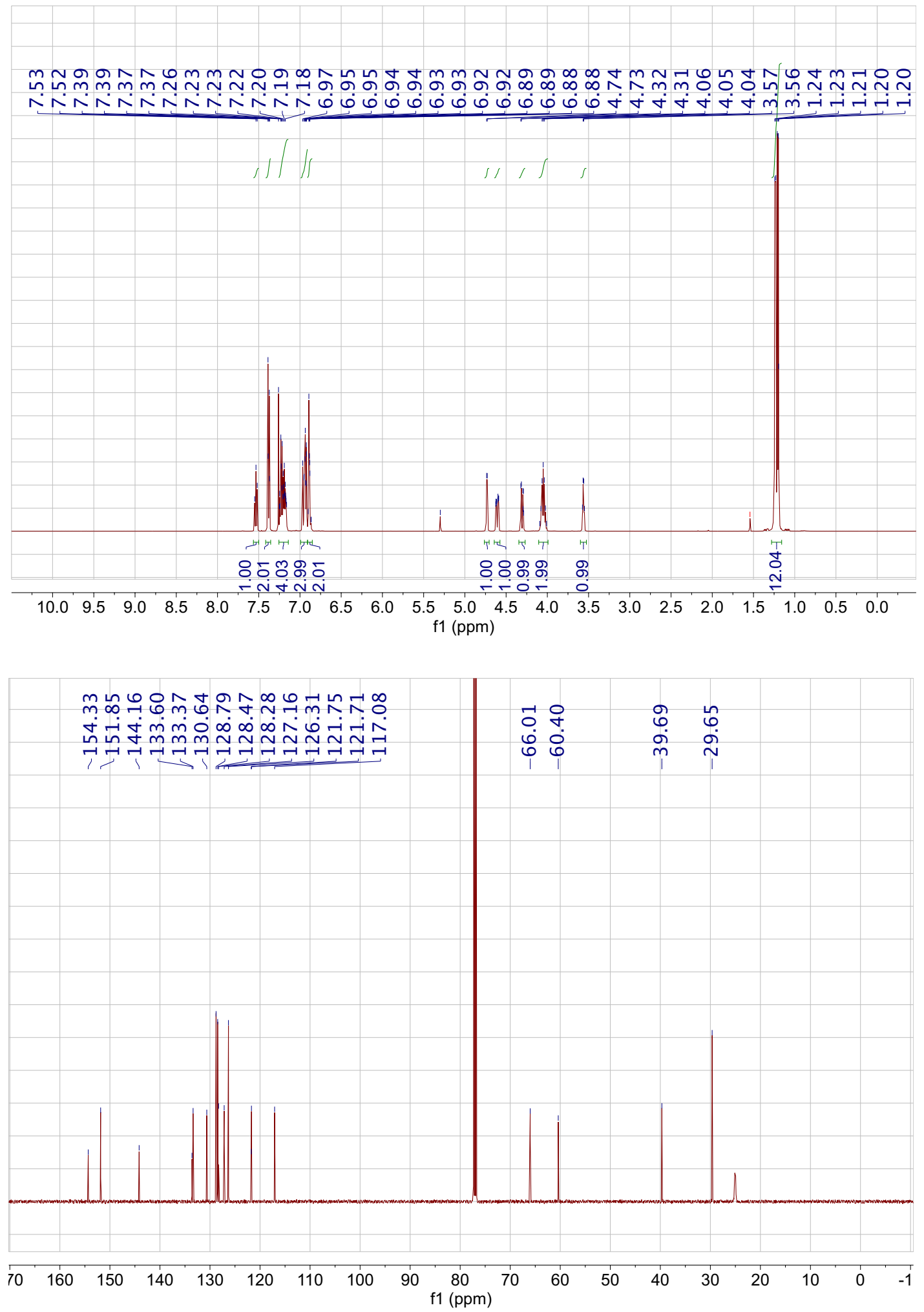
(3S,4R)-4-Phenyl-3-(phenylthio)chromane - (10) - ${ }^{1} \mathrm{H}$ NMR $\left(500 \mathrm{MHz}, \mathrm{CDCl}_{3}\right) ;{ }^{13} \mathrm{C}\left\{{ }^{1} \mathrm{H}\right\}(126$ $\mathrm{MHz}, \mathrm{CDCl}_{3}$ )
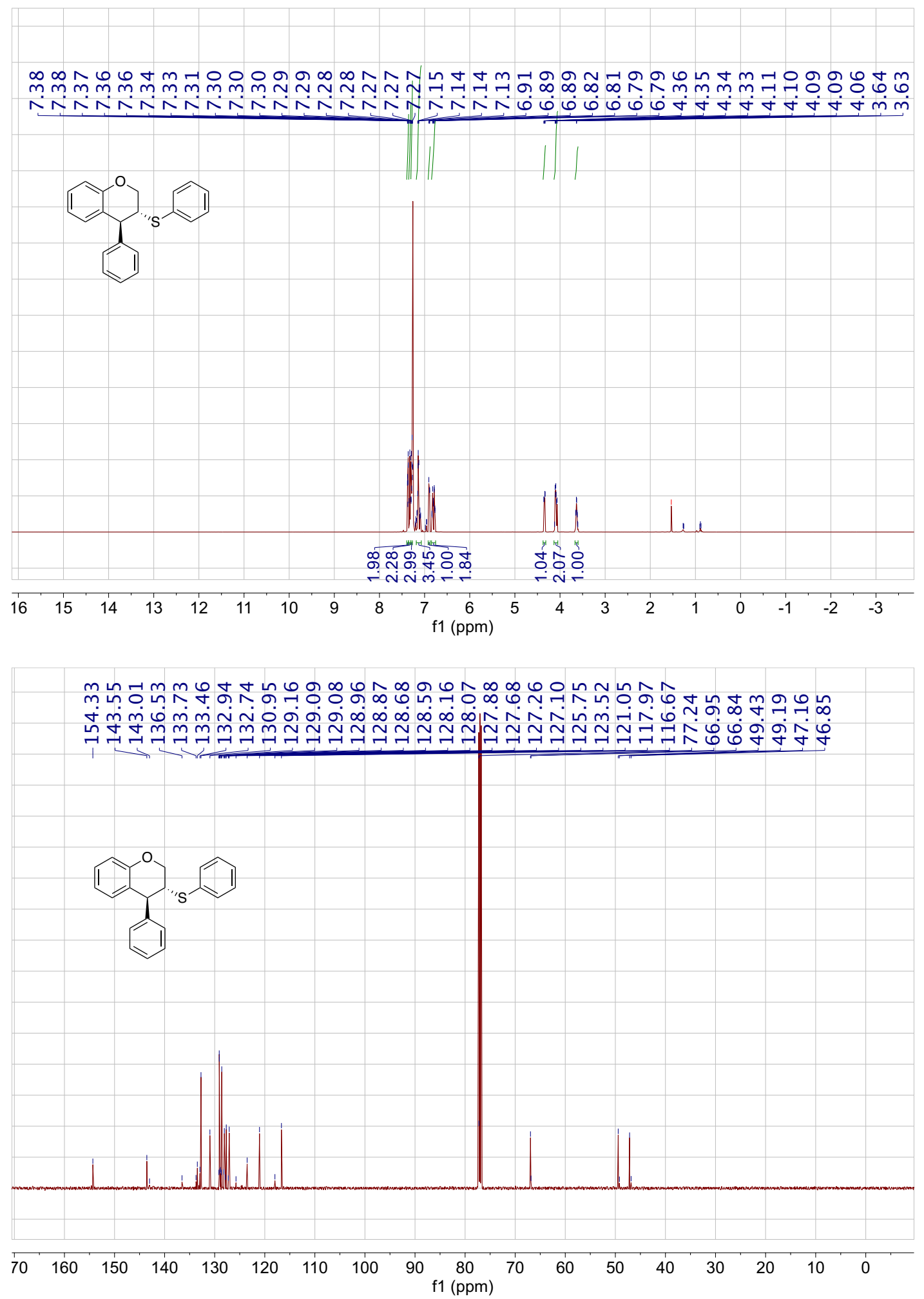


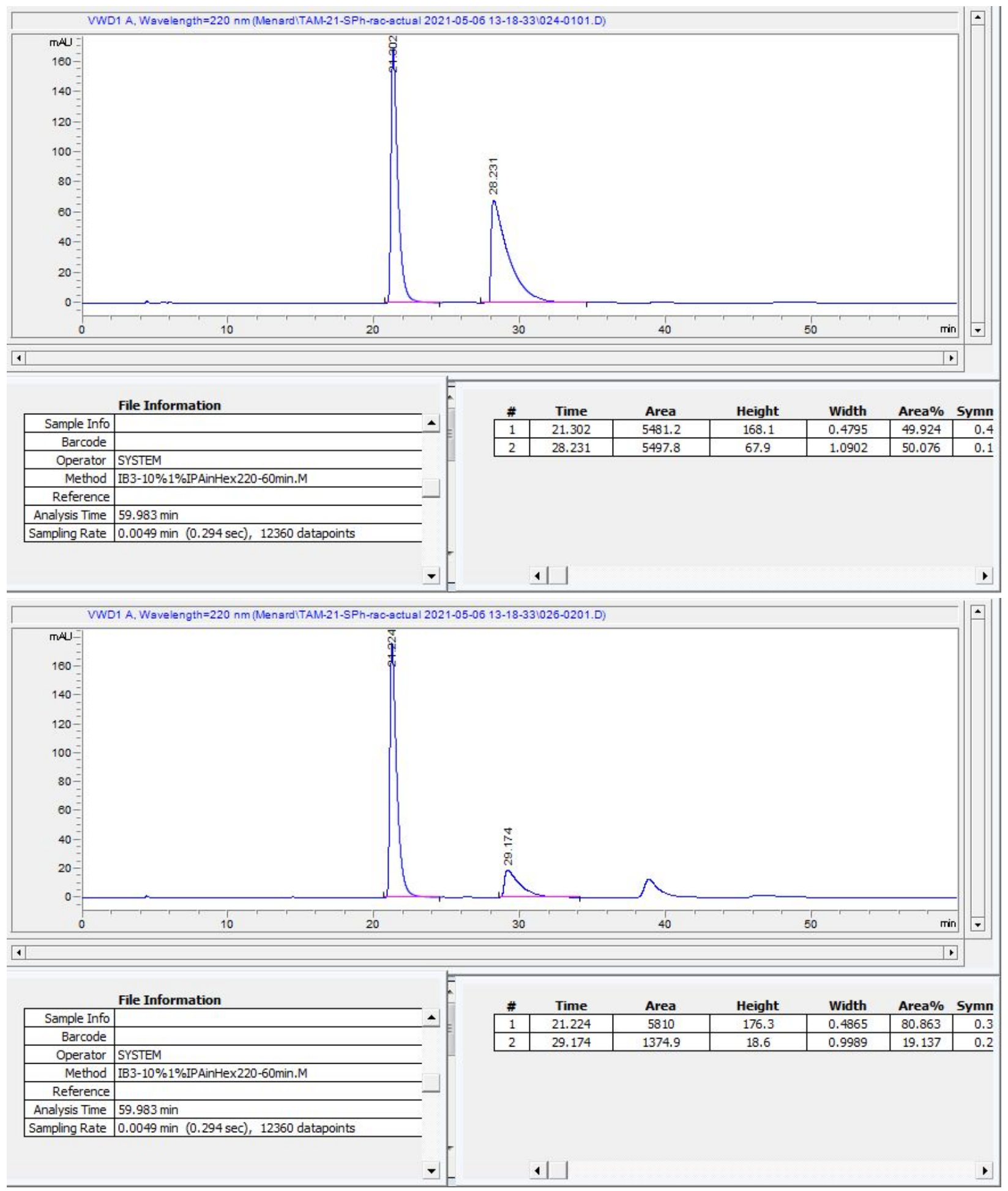


(3R,4S)-4-Phenylchromane-3-thiol - (9) - ${ }^{1} \mathrm{H}$ NMR $\left(500 \mathrm{MHz}, \mathrm{CDCl}_{3}\right) ;{ }^{13} \mathrm{C}\left\{{ }^{1} \mathrm{H}\right\}(126 \mathrm{MHz}$, $\left.\mathrm{CDCl}_{3}\right)$
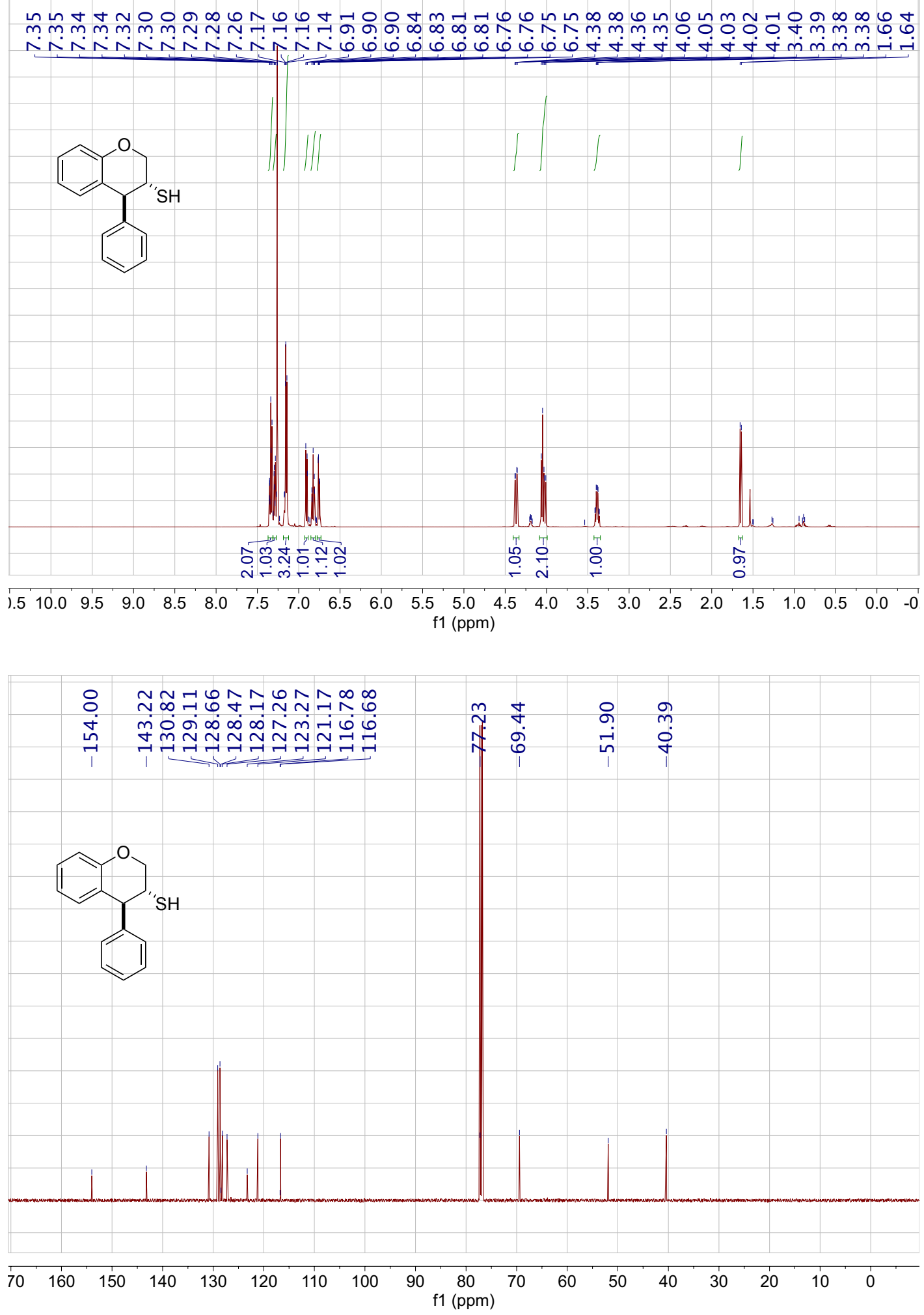
( \pm )-1-Phenyl-5-(((3R,4S)-4-phenylchroman-3-yl)thio)-1H-tetrazole (12) - ${ }^{1} \mathrm{H}$ NMR $(500 \mathrm{MHz}$, $\left.\mathrm{CDCl}_{3}\right) ;{ }^{13} \mathrm{C}\left\{{ }^{1} \mathrm{H}\right\}\left(126 \mathrm{MHz}, \mathrm{CDCl}_{3}\right)$
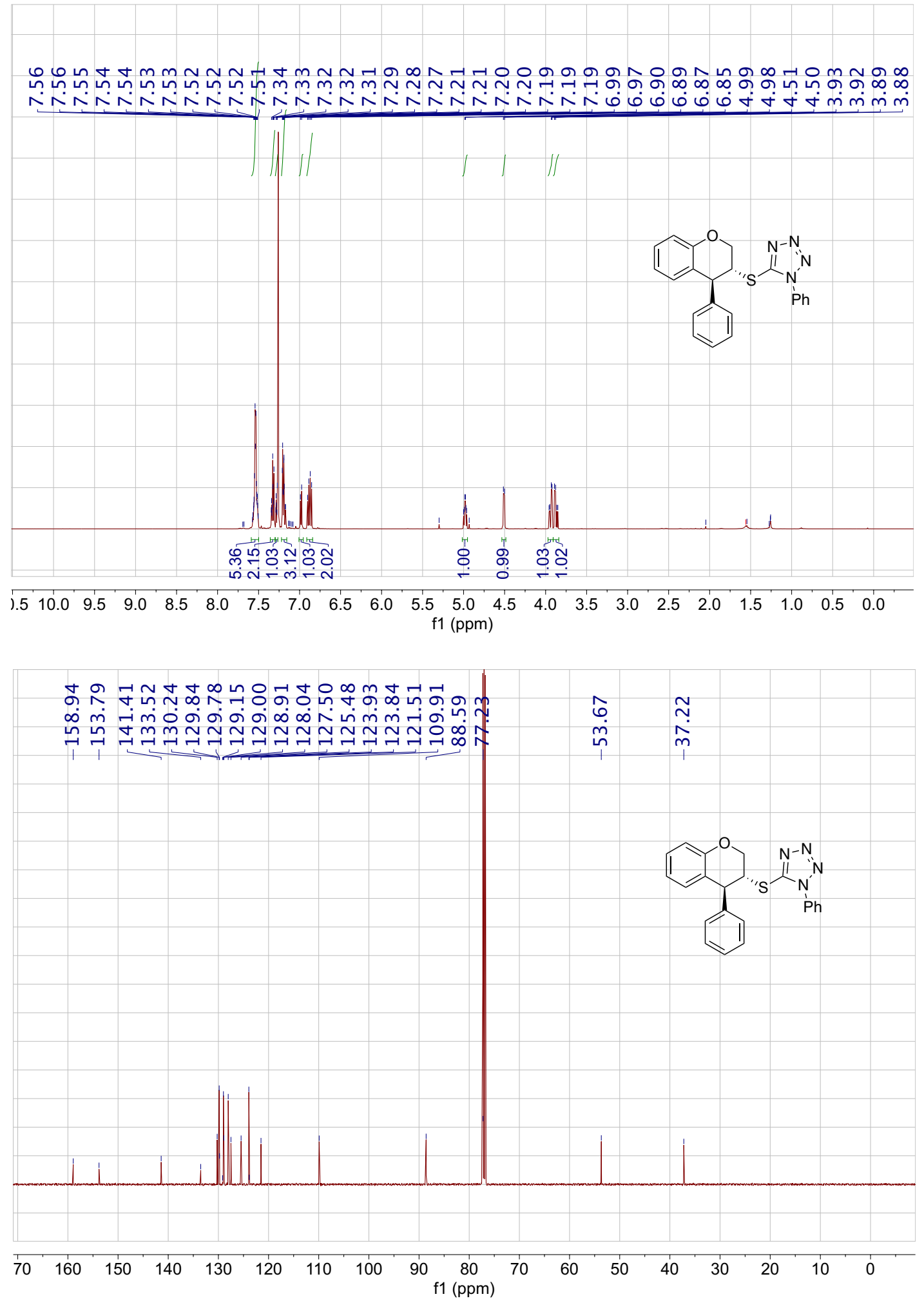
( \pm )-1-phenyl-5-(((3R,4S)-4-phenylchroman-3-yl)sulfonyl)-1H-tetrazole (13) - ${ }^{1} \mathrm{H}$ NMR (500 $\left.\mathrm{MHz}, \mathrm{CDCl}_{3}\right) ;{ }^{13} \mathrm{C}\left\{{ }^{1} \mathrm{H}\right\}\left(126 \mathrm{MHz}, \mathrm{CDCl}_{3}\right)$

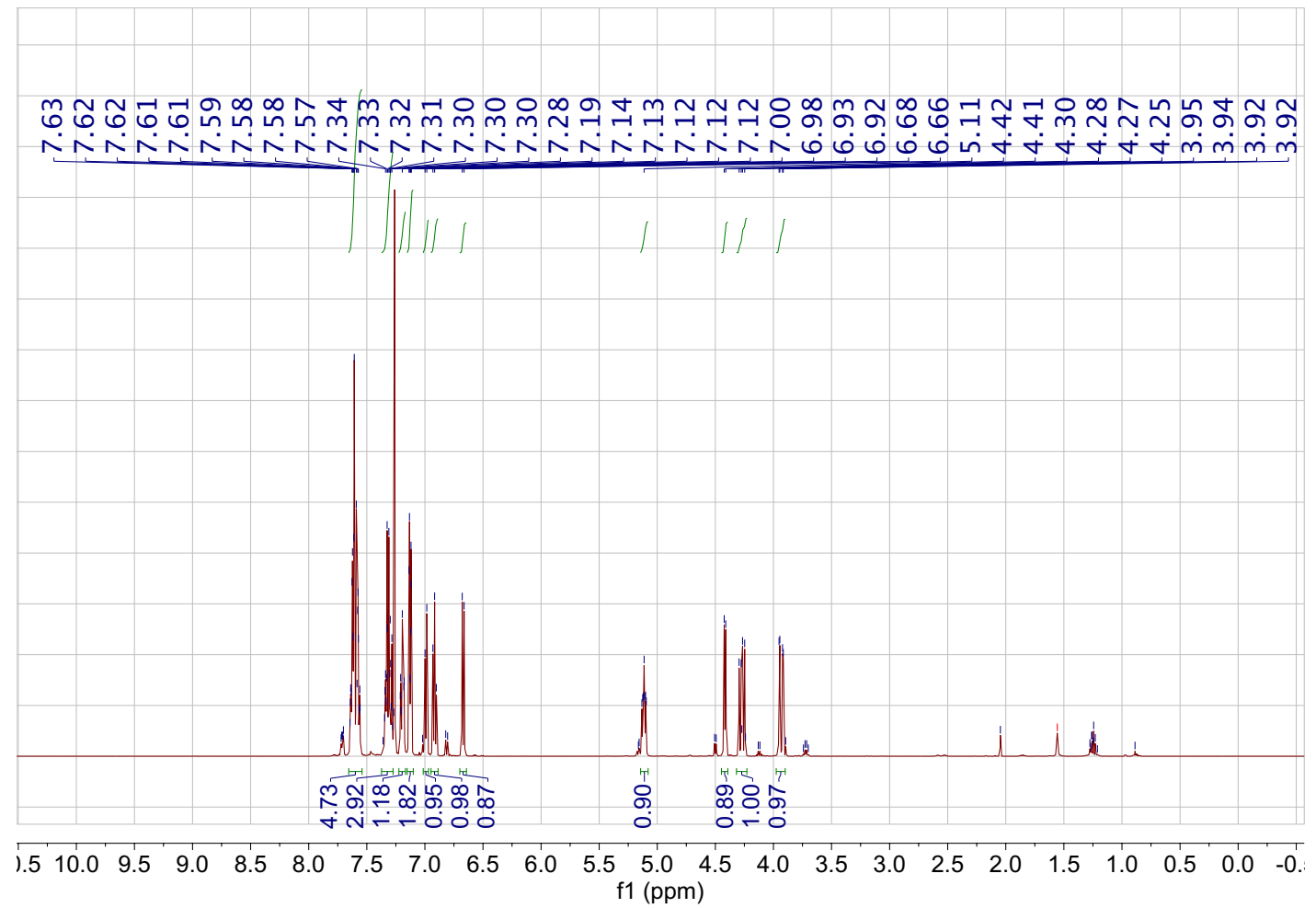

Fのm

めர்

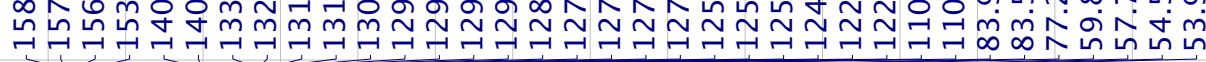

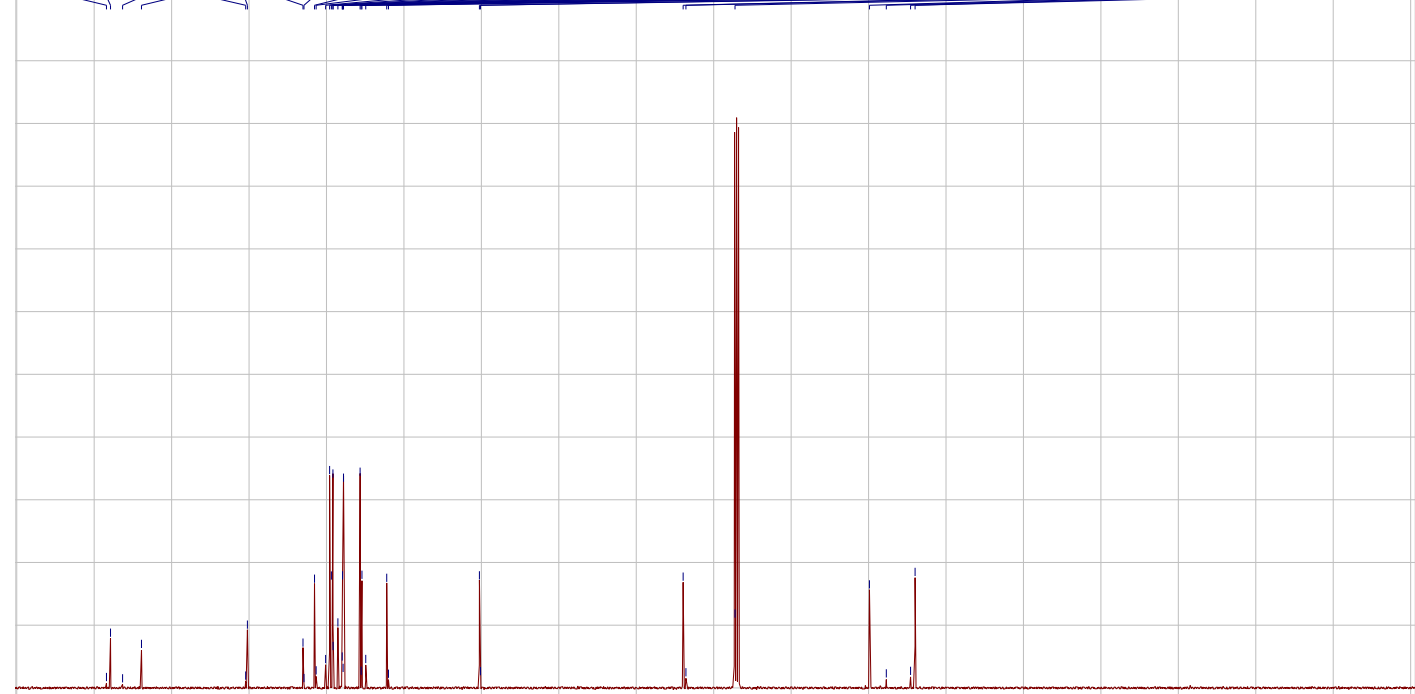

$\begin{array}{llllllllllllllllllll}70 & 160 & 150 & 140 & 130 & 120 & 110 & 100 & 90 & \begin{array}{c}80 \\ \mathrm{f} 1(\mathrm{ppm})\end{array} & 70 & 60 & 50 & 40 & 30 & 20 & 10 & 0 & -1\end{array}$ 


\section{X-ray - Determination of Absolute Configuration}

The following X-ray structure was obtained from the cyclization of substrate 1e utilizing the BINOL catalyst $(R)-6$ furnishing $(3 R, 4 S)$-3e (ent-3e). The sample was prepared by dissolving completely in minimal HPLC grade hexanes and allowing for slow evaporation at $23{ }^{\circ} \mathrm{C}$ to yield thin needles.

Substrate $(3 S, 4 R)-3 \mathbf{e}$ in the main text was synthesized using catalyst $(S)-\mathbf{5}$. The absolute configuration of ent-3e , as determined by X-ray structure, allows for the assignment of absolute configuration of all products cyclized by analogy. CSP-HPLC UV traces reveal that the opposite enantiomer is furnished when using $(S)-5$ versus $(R)-6$.

\section{Crystallography}

Intensity data were collected on a Bruker D8 Venture kappa diffractometer equipped with a Photon II detector. An I $\mu$ s microfocus source provided the Mo K $\alpha$ radiation $(\lambda=0,71073 \AA)$ that was monochromated with multilayer mirrors. The collection, cell refinement and integration of intensity data were carried out with the APEX3 software (1). The absorption corrections were performed using multi-scan methods with SADABS (2). The initial structure solution was solved with dual space methods using SHELXT (3), and the structure was refined with the full-matrix least-squares SHELXL (4) program.

X-ray Data for $(3 R, 4 S)$-6-Bromo-3-((2,6-diisopropylphenyl)thio)-4-phenylchromane - (ent-3e)

Crystal Data and Structure Refinement.

Identification code

Empirical formula

Formula weight

Temperature

Wavelength

Crystal system

Space group

Unit cell dimensions

Volume
CCDC 2105042

C27 H29 Br O S

481.47

130(2) K

$0.71073 \AA$

Monoclinic

P21

$\mathrm{a}=11.9201(3) \AA \quad \mathrm{a}=90^{\circ}$.

$\mathrm{b}=5.76180(10) \AA$

$b=100.1760(8)^{\circ}$.

$\mathrm{c}=17.3524(4) \AA$

$g=90^{\circ}$.

1173.04(5) $\AA^{3}$ 
Z

Density (calculated)

Absorption coefficient

$\mathrm{F}(000)$

Crystal size

Theta range for data collection

Index ranges

Reflections collected

Independent reflections

Completeness to theta $=25.242^{\circ}$

Absorption correction

Max. and min. transmission

Refinement method

Data / restraints / parameters

Goodness-of-fit on $\mathrm{F}^{2}$

Final $\mathrm{R}$ indices [I $>2 \operatorname{sigma}(\mathrm{I})]$

$\mathrm{R}$ indices (all data)

Absolute structure parameter

Extinction coefficient

Largest diff. peak and hole
2

$1.363 \mathrm{Mg} / \mathrm{m}^{3}$

$1.857 \mathrm{~mm}^{-1}$

500

$0.328 \times 0.138 \times 0.072 \mathrm{~mm}^{3}$

2.273 to $28.294^{\circ}$.

$-15<=\mathrm{h}<=15,-7<=\mathrm{k}<=7,-22<=\mathrm{l}<=23$

41853

$5821[\mathrm{R}(\mathrm{int})=0.0402]$

$99.9 \%$

Semi-empirical from equivalents

0.7457 and 0.6343

Full-matrix least-squares on $\mathrm{F}^{2}$

$5821 / 1 / 275$

1.048

$\mathrm{R} 1=0.0252, \mathrm{wR} 2=0.0523$

$\mathrm{R} 1=0.0305, \mathrm{wR} 2=0.0547$

$-0.007(2)$

$\mathrm{n} / \mathrm{a}$

0.266 and -0.302 e. $\AA^{-3}$ 
Figure S2. X-Ray structure of $(3 R, 4 S)$-3e (35\% ellipsoid contour probability)

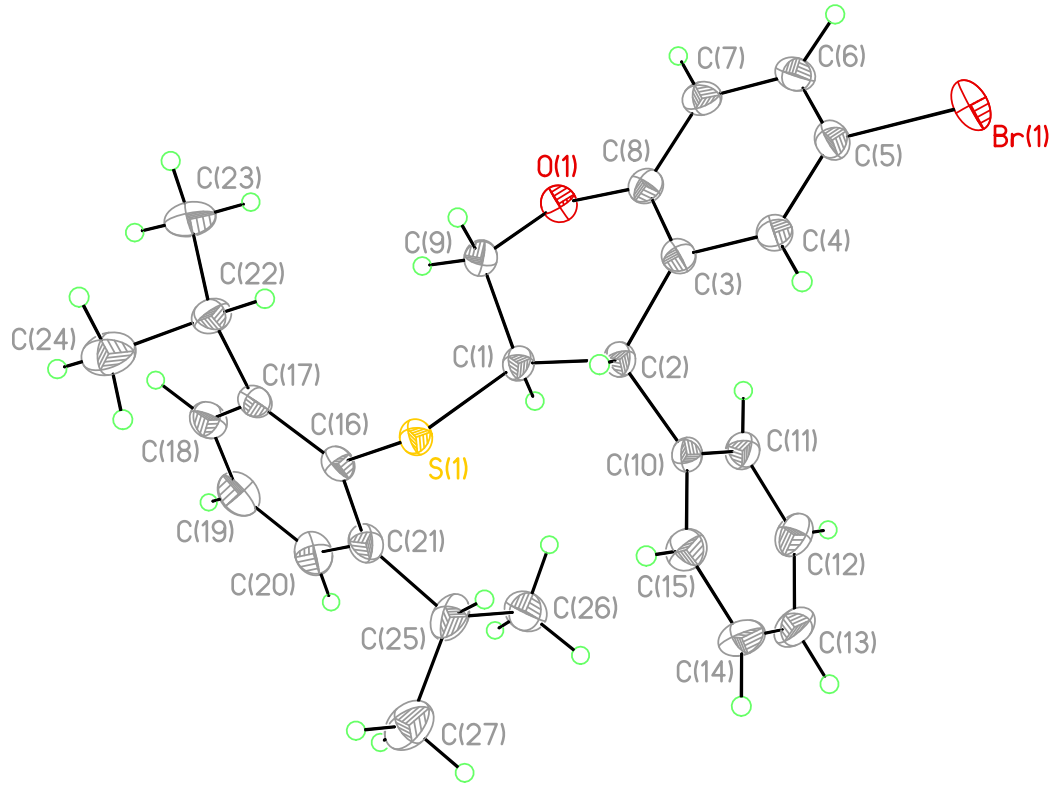

Table S1. Atomic coordinates ( $\mathrm{x} 10^{4}$ ) and equivalent isotropic displacement parameters $\left(\AA^{2} \mathrm{x} 10^{3}\right)$ for ed61m1s. $U(e q)$ is defined as one third of the trace of the orthogonalized $U^{\mathrm{ij}}$ tensor.

\begin{tabular}{lcccc}
\hline & $\mathrm{x}$ & $\mathrm{y}$ & $\mathrm{z}$ & $\mathrm{U}(\mathrm{eq})$ \\
\hline $\mathrm{Br}(1)$ & $9162(1)$ & $7485(1)$ & $9495(1)$ & $34(1)$ \\
$\mathrm{S}(1)$ & $3267(1)$ & $6782(1)$ & $6534(1)$ & $20(1)$ \\
$\mathrm{O}(1)$ & $5721(1)$ & $1973(3)$ & $7254(1)$ & $23(1)$ \\
$\mathrm{C}(1)$ & $4230(2)$ & $4883(4)$ & $7191(1)$ & $19(1)$ \\
$\mathrm{C}(2)$ & $4984(2)$ & $6430(4)$ & $7808(1)$ & $18(1)$ \\
$\mathrm{C}(3)$ & $6174(2)$ & $5420(4)$ & $8040(1)$ & $20(1)$ \\
$\mathrm{C}(4)$ & $6992(2)$ & $6655(5)$ & $8557(1)$ & $22(1)$ \\
$\mathrm{C}(5)$ & $8065(2)$ & $5727(5)$ & $8801(2)$ & $24(1)$ \\
$\mathrm{C}(6)$ & $8359(2)$ & $3565(5)$ & $8549(2)$ & $26(1)$ \\
$\mathrm{C}(7)$ & $7561(2)$ & $2345(6)$ & $8024(1)$ & $24(1)$ \\
$\mathrm{C}(8)$ & $6483(2)$ & $3273(4)$ & $7774(1)$ & $21(1)$ \\
$\mathrm{C}(9)$ & $4930(2)$ & $3402(4)$ & $6735(2)$ & $22(1)$
\end{tabular}




\begin{tabular}{lrlll}
$\mathrm{C}(10)$ & $4392(2)$ & $6716(4)$ & $8512(1)$ & $20(1)$ \\
$\mathrm{C}(11)$ & $4450(2)$ & $4947(5)$ & $9064(2)$ & $24(1)$ \\
$\mathrm{C}(12)$ & $3842(3)$ & $5100(5)$ & $9676(2)$ & $30(1)$ \\
$\mathrm{C}(13)$ & $3171(2)$ & $7015(5)$ & $9743(2)$ & $32(1)$ \\
$\mathrm{C}(14)$ & $3113(3)$ & $8796(5)$ & $9202(2)$ & $34(1)$ \\
$\mathrm{C}(15)$ & $3724(2)$ & $8645(5)$ & $8589(2)$ & $28(1)$ \\
$\mathrm{C}(16)$ & $2233(2)$ & $4657(4)$ & $6123(2)$ & $21(1)$ \\
$\mathrm{C}(17)$ & $2305(2)$ & $3625(4)$ & $5398(2)$ & $22(1)$ \\
$\mathrm{C}(18)$ & $1539(2)$ & $1862(5)$ & $5126(2)$ & $27(1)$ \\
$\mathrm{C}(19)$ & $717(2)$ & $1159(5)$ & $5548(2)$ & $33(1)$ \\
$\mathrm{C}(20)$ & $634(2)$ & $2245(6)$ & $6244(2)$ & $32(1)$ \\
$\mathrm{C}(21)$ & $1376(2)$ & $4021(5)$ & $6547(2)$ & $27(1)$ \\
$\mathrm{C}(22)$ & $3138(2)$ & $4439(5)$ & $4880(2)$ & $28(1)$ \\
$\mathrm{C}(23)$ & $3737(2)$ & $2443(8)$ & $4542(2)$ & $44(1)$ \\
$\mathrm{C}(24)$ & $2508(3)$ & $5952(6)$ & $4217(2)$ & $40(1)$ \\
$\mathrm{C}(25)$ & $1209(3)$ & $5186(6)$ & $7306(2)$ & $36(1)$ \\
$\mathrm{C}(26)$ & $1321(3)$ & $3457(8)$ & $7981(2)$ & $54(1)$ \\
$\mathrm{C}(27)$ & $53(3)$ & $6409(6)$ & $7204(2)$ & $44(1)$ \\
& & & & \\
\hline
\end{tabular}


Table S2. Bond lengths $[\AA]$ and angles $\left[{ }^{\circ}\right]$ for ed61m1s.

\begin{tabular}{|c|c|}
\hline $\operatorname{Br}(1)-C(5)$ & $1.905(3)$ \\
\hline$S(1)-C(16)$ & 1.793(3) \\
\hline $\mathrm{S}(1)-\mathrm{C}(1)$ & $1.831(3)$ \\
\hline $\mathrm{O}(1)-\mathrm{C}(8)$ & $1.382(3)$ \\
\hline $\mathrm{O}(1)-\mathrm{C}(9)$ & $1.442(3)$ \\
\hline $\mathrm{C}(1)-\mathrm{C}(9)$ & $1.511(3)$ \\
\hline $\mathrm{C}(1)-\mathrm{C}(2)$ & $1.552(3)$ \\
\hline $\mathrm{C}(1)-\mathrm{H}(1)$ & 1.0000 \\
\hline $\mathrm{C}(2)-\mathrm{C}(3)$ & $1.520(3)$ \\
\hline $\mathrm{C}(2)-\mathrm{C}(10)$ & $1.524(3)$ \\
\hline $\mathrm{C}(2)-\mathrm{H}(2)$ & 1.0000 \\
\hline $\mathrm{C}(3)-\mathrm{C}(8)$ & $1.393(3)$ \\
\hline $\mathrm{C}(3)-\mathrm{C}(4)$ & $1.398(3)$ \\
\hline$C(4)-C(5)$ & $1.382(3)$ \\
\hline $\mathrm{C}(4)-\mathrm{H}(4)$ & 0.9500 \\
\hline$C(5)-C(6)$ & $1.386(4)$ \\
\hline$C(6)-C(7)$ & $1.386(4)$ \\
\hline $\mathrm{C}(6)-\mathrm{H}(6)$ & 0.9500 \\
\hline$C(7)-C(8)$ & $1.390(3)$ \\
\hline $\mathrm{C}(7)-\mathrm{H}(7)$ & 0.9500 \\
\hline $\mathrm{C}(9)-\mathrm{H}(9 \mathrm{~A})$ & 0.9900 \\
\hline $\mathrm{C}(9)-\mathrm{H}(9 \mathrm{~B})$ & 0.9900 \\
\hline$C(10)-C(15)$ & $1.386(3)$ \\
\hline$C(10)-C(11)$ & $1.391(3)$ \\
\hline$C(11)-C(12)$ & $1.390(4)$ \\
\hline $\mathrm{C}(11)-\mathrm{H}(11)$ & 0.9500 \\
\hline$C(12)-C(13)$ & $1.380(4)$ \\
\hline $\mathrm{C}(12)-\mathrm{H}(12)$ & 0.9500 \\
\hline$C(13)-C(14)$ & $1.384(4)$ \\
\hline $\mathrm{C}(13)-\mathrm{H}(13)$ & 0.9500 \\
\hline$C(14)-C(15)$ & $1.395(4)$ \\
\hline $\mathrm{C}(14)-\mathrm{H}(14)$ & 0.9500 \\
\hline $\mathrm{C}(15)-\mathrm{H}(15)$ & 0.9500 \\
\hline$C(16)-C(17)$ & $1.408(4)$ \\
\hline
\end{tabular}




\begin{tabular}{|c|c|}
\hline$C(16)-C(21)$ & $1.409(4)$ \\
\hline$C(17)-C(18)$ & $1.392(4)$ \\
\hline$C(17)-C(22)$ & $1.526(4)$ \\
\hline $\mathrm{C}(18)-\mathrm{C}(19)$ & $1.383(4)$ \\
\hline $\mathrm{C}(18)-\mathrm{H}(18)$ & 0.9500 \\
\hline$C(19)-C(20)$ & $1.380(4)$ \\
\hline $\mathrm{C}(19)-\mathrm{H}(19)$ & 0.9500 \\
\hline$C(20)-C(21)$ & $1.394(4)$ \\
\hline $\mathrm{C}(20)-\mathrm{H}(20)$ & 0.9500 \\
\hline$C(21)-C(25)$ & $1.523(4)$ \\
\hline$C(22)-C(23)$ & $1.524(4)$ \\
\hline$C(22)-C(24)$ & $1.530(4)$ \\
\hline $\mathrm{C}(22)-\mathrm{H}(22)$ & 1.0000 \\
\hline $\mathrm{C}(23)-\mathrm{H}(23 \mathrm{~A})$ & 0.9800 \\
\hline $\mathrm{C}(23)-\mathrm{H}(23 \mathrm{~B})$ & 0.9800 \\
\hline $\mathrm{C}(23)-\mathrm{H}(23 \mathrm{C})$ & 0.9800 \\
\hline $\mathrm{C}(24)-\mathrm{H}(24 \mathrm{~A})$ & 0.9800 \\
\hline $\mathrm{C}(24)-\mathrm{H}(24 \mathrm{~B})$ & 0.9800 \\
\hline $\mathrm{C}(24)-\mathrm{H}(24 \mathrm{C})$ & 0.9800 \\
\hline$C(25)-C(26)$ & $1.525(5)$ \\
\hline$C(25)-C(27)$ & $1.530(5)$ \\
\hline $\mathrm{C}(25)-\mathrm{H}(25)$ & 1.0000 \\
\hline $\mathrm{C}(26)-\mathrm{H}(26 \mathrm{~A})$ & 0.9800 \\
\hline $\mathrm{C}(26)-\mathrm{H}(26 \mathrm{~B})$ & 0.9800 \\
\hline $\mathrm{C}(26)-\mathrm{H}(26 \mathrm{C})$ & 0.9800 \\
\hline $\mathrm{C}(27)-\mathrm{H}(27 \mathrm{~A})$ & 0.9800 \\
\hline $\mathrm{C}(27)-\mathrm{H}(27 \mathrm{~B})$ & 0.9800 \\
\hline $\mathrm{C}(27)-\mathrm{H}(27 \mathrm{C})$ & 0.9800 \\
\hline$C(16)-S(1)-C(1)$ & $98.68(12)$ \\
\hline $\mathrm{C}(8)-\mathrm{O}(1)-\mathrm{C}(9)$ & $112.37(18)$ \\
\hline $\mathrm{C}(9)-\mathrm{C}(1)-\mathrm{C}(2)$ & $112.3(2)$ \\
\hline $\mathrm{C}(9)-\mathrm{C}(1)-\mathrm{S}(1)$ & $110.77(17)$ \\
\hline$C(2)-C(1)-S(1)$ & $107.99(16)$ \\
\hline $\mathrm{C}(9)-\mathrm{C}(1)-\mathrm{H}(1)$ & 108.6 \\
\hline $\mathrm{C}(2)-\mathrm{C}(1)-\mathrm{H}(1)$ & 108.6 \\
\hline
\end{tabular}




\begin{tabular}{|c|c|}
\hline$S(1)-C(1)-H(1)$ & 108.6 \\
\hline$C(3)-C(2)-C(10)$ & $111.8(2)$ \\
\hline$C(3)-C(2)-C(1)$ & $111.2(2)$ \\
\hline$C(10)-C(2)-C(1)$ & $108.77(19)$ \\
\hline $\mathrm{C}(3)-\mathrm{C}(2)-\mathrm{H}(2)$ & 108.3 \\
\hline $\mathrm{C}(10)-\mathrm{C}(2)-\mathrm{H}(2)$ & 108.3 \\
\hline $\mathrm{C}(1)-\mathrm{C}(2)-\mathrm{H}(2)$ & 108.3 \\
\hline $\mathrm{C}(8)-\mathrm{C}(3)-\mathrm{C}(4)$ & $118.0(2)$ \\
\hline $\mathrm{C}(8)-\mathrm{C}(3)-\mathrm{C}(2)$ & $122.8(2)$ \\
\hline $\mathrm{C}(4)-\mathrm{C}(3)-\mathrm{C}(2)$ & 119.2(2) \\
\hline$C(5)-C(4)-C(3)$ & $120.2(2)$ \\
\hline $\mathrm{C}(5)-\mathrm{C}(4)-\mathrm{H}(4)$ & 119.9 \\
\hline $\mathrm{C}(3)-\mathrm{C}(4)-\mathrm{H}(4)$ & 119.9 \\
\hline$C(4)-C(5)-C(6)$ & $121.5(2)$ \\
\hline$C(4)-C(5)-B r(1)$ & $118.9(2)$ \\
\hline$C(6)-C(5)-B r(1)$ & 119.61(19) \\
\hline$C(5)-C(6)-C(7)$ & $118.8(2)$ \\
\hline $\mathrm{C}(5)-\mathrm{C}(6)-\mathrm{H}(6)$ & 120.6 \\
\hline $\mathrm{C}(7)-\mathrm{C}(6)-\mathrm{H}(6)$ & 120.6 \\
\hline $\mathrm{C}(6)-\mathrm{C}(7)-\mathrm{C}(8)$ & $120.0(3)$ \\
\hline $\mathrm{C}(6)-\mathrm{C}(7)-\mathrm{H}(7)$ & 120.0 \\
\hline $\mathrm{C}(8)-\mathrm{C}(7)-\mathrm{H}(7)$ & 120.0 \\
\hline $\mathrm{O}(1)-\mathrm{C}(8)-\mathrm{C}(7)$ & $117.4(2)$ \\
\hline $\mathrm{O}(1)-\mathrm{C}(8)-\mathrm{C}(3)$ & 121.1(2) \\
\hline $\mathrm{C}(7)-\mathrm{C}(8)-\mathrm{C}(3)$ & $121.5(2)$ \\
\hline $\mathrm{O}(1)-\mathrm{C}(9)-\mathrm{C}(1)$ & $110.8(2)$ \\
\hline $\mathrm{O}(1)-\mathrm{C}(9)-\mathrm{H}(9 \mathrm{~A})$ & 109.5 \\
\hline $\mathrm{C}(1)-\mathrm{C}(9)-\mathrm{H}(9 \mathrm{~A})$ & 109.5 \\
\hline $\mathrm{O}(1)-\mathrm{C}(9)-\mathrm{H}(9 \mathrm{~B})$ & 109.5 \\
\hline $\mathrm{C}(1)-\mathrm{C}(9)-\mathrm{H}(9 \mathrm{~B})$ & 109.5 \\
\hline $\mathrm{H}(9 \mathrm{~A})-\mathrm{C}(9)-\mathrm{H}(9 \mathrm{~B})$ & 108.1 \\
\hline $\mathrm{C}(15)-\mathrm{C}(10)-\mathrm{C}(11)$ & $118.8(2)$ \\
\hline$C(15)-C(10)-C(2)$ & $121.2(2)$ \\
\hline $\mathrm{C}(11)-\mathrm{C}(10)-\mathrm{C}(2)$ & $119.9(2)$ \\
\hline $\mathrm{C}(12)-\mathrm{C}(11)-\mathrm{C}(10)$ & $120.6(2)$ \\
\hline $\mathrm{C}(12)-\mathrm{C}(11)-\mathrm{H}(11)$ & 119.7 \\
\hline
\end{tabular}




$\begin{array}{ll}\mathrm{C}(10)-\mathrm{C}(11)-\mathrm{H}(11) & 119.7 \\ \mathrm{C}(13)-\mathrm{C}(12)-\mathrm{C}(11) & 120.2(3) \\ \mathrm{C}(13)-\mathrm{C}(12)-\mathrm{H}(12) & 119.9 \\ \mathrm{C}(11)-\mathrm{C}(12)-\mathrm{H}(12) & 119.9 \\ \mathrm{C}(12)-\mathrm{C}(13)-\mathrm{C}(14) & 119.7(2) \\ \mathrm{C}(12)-\mathrm{C}(13)-\mathrm{H}(13) & 120.1 \\ \mathrm{C}(14)-\mathrm{C}(13)-\mathrm{H}(13) & 120.1 \\ \mathrm{C}(13)-\mathrm{C}(14)-\mathrm{C}(15) & 120.0(3) \\ \mathrm{C}(13)-\mathrm{C}(14)-\mathrm{H}(14) & 120.0 \\ \mathrm{C}(15)-\mathrm{C}(14)-\mathrm{H}(14) & 120.0 \\ \mathrm{C}(10)-\mathrm{C}(15)-\mathrm{C}(14) & 120.6(3) \\ \mathrm{C}(10)-\mathrm{C}(15)-\mathrm{H}(15) & 119.7 \\ \mathrm{C}(14)-\mathrm{C}(15)-\mathrm{H}(15) & 119.7 \\ \mathrm{C}(17)-\mathrm{C}(16)-\mathrm{C}(21) & 121.4(2) \\ \mathrm{C}(17)-\mathrm{C}(16)-\mathrm{S}(1) & 119.86(19) \\ \mathrm{C}(21)-\mathrm{C}(16)-\mathrm{S}(1) & 118.7(2) \\ \mathrm{C}(18)-\mathrm{C}(17)-\mathrm{C}(16) & 118.1(2) \\ \mathrm{C}(18)-\mathrm{C}(17)-\mathrm{C}(22) & 118.7(2) \\ \mathrm{C}(16)-\mathrm{C}(17)-\mathrm{C}(22) & 123.0(2) \\ \mathrm{C}(19)-\mathrm{C}(18)-\mathrm{C}(17) & 121.2(3) \\ \mathrm{C}(19)-\mathrm{C}(18)-\mathrm{H}(18) & 119.4 \\ \mathrm{C}(17)-\mathrm{C}(18)-\mathrm{H}(18) & 119.4 \\ \mathrm{C}(20)-\mathrm{C}(19)-\mathrm{C}(18) & 119.8(2) \\ \mathrm{C}(20)-\mathrm{C}(19)-\mathrm{H}(19) & 120.1 \\ \mathrm{C}(18)-\mathrm{C}(19)-\mathrm{H}(19) & 120.1 \\ \mathrm{C}(19)-\mathrm{C}(20)-\mathrm{C}(21) & 121.7(2) \\ \mathrm{C}(19)-\mathrm{C}(20)-\mathrm{H}(20) & 119.2 \\ \mathrm{C}(21)-\mathrm{C}(20)-\mathrm{H}(20) & 119.2 \\ \mathrm{C}(20)-\mathrm{C}(21)-\mathrm{C}(16) & 117.7(3) \\ \mathrm{C}(20)-\mathrm{C}(21)-\mathrm{C}(25) & 118.6(2) \\ \mathrm{C}(16)-\mathrm{C}(21)-\mathrm{C}(25) & 123.7(2) \\ \mathrm{C}(23)-\mathrm{C}(22)-\mathrm{C}(17) & 113.1(3) \\ \mathrm{C}(23)-\mathrm{C}(22)-\mathrm{C}(24) & 110.0(2) \\ & 109.5(2) \\ \mathrm{C}(22)-\mathrm{C}(24) & 108.0 \\ & \end{array}$




$\begin{array}{ll}\mathrm{C}(24)-\mathrm{C}(22)-\mathrm{H}(22) & 108.0 \\ \mathrm{C}(22)-\mathrm{C}(23)-\mathrm{H}(23 \mathrm{~A}) & 109.5 \\ \mathrm{C}(22)-\mathrm{C}(23)-\mathrm{H}(23 \mathrm{~B}) & 109.5 \\ \mathrm{H}(23 \mathrm{~A})-\mathrm{C}(23)-\mathrm{H}(23 \mathrm{~B}) & 109.5 \\ \mathrm{C}(22)-\mathrm{C}(23)-\mathrm{H}(23 \mathrm{C}) & 109.5 \\ \mathrm{H}(23 \mathrm{~A})-\mathrm{C}(23)-\mathrm{H}(23 \mathrm{C}) & 109.5 \\ \mathrm{H}(23 \mathrm{~B})-\mathrm{C}(23)-\mathrm{H}(23 \mathrm{C}) & 109.5 \\ \mathrm{C}(22)-\mathrm{C}(24)-\mathrm{H}(24 \mathrm{~A}) & 109.5 \\ \mathrm{C}(22)-\mathrm{C}(24)-\mathrm{H}(24 \mathrm{~B}) & 109.5 \\ \mathrm{H}(24 \mathrm{~A})-\mathrm{C}(24)-\mathrm{H}(24 \mathrm{~B}) & 109.5 \\ \mathrm{C}(22)-\mathrm{C}(24)-\mathrm{H}(24 \mathrm{C}) & 109.5 \\ \mathrm{H}(24 \mathrm{~A})-\mathrm{C}(24)-\mathrm{H}(24 \mathrm{C}) & 109.5 \\ \mathrm{H}(24 \mathrm{~B})-\mathrm{C}(24)-\mathrm{H}(24 \mathrm{C}) & 109.5 \\ \mathrm{C}(21)-\mathrm{C}(25)-\mathrm{C}(26) & 111.6(3) \\ \mathrm{C}(21)-\mathrm{C}(25)-\mathrm{C}(27) & 110.8(3) \\ \mathrm{C}(26)-\mathrm{C}(25)-\mathrm{C}(27) & 110.2(2) \\ \mathrm{C}(21)-\mathrm{C}(25)-\mathrm{H}(25) & 108.0 \\ \mathrm{C}(26)-\mathrm{C}(25)-\mathrm{H}(25) & 108.0 \\ \mathrm{C}(27)-\mathrm{C}(25)-\mathrm{H}(25) & 108.0 \\ \mathrm{C}(25)-\mathrm{C}(26)-\mathrm{H}(26 \mathrm{~A}) & 109.5 \\ \mathrm{C}(25)-\mathrm{C}(26)-\mathrm{H}(26 \mathrm{~B}) & 109.5 \\ \mathrm{H}(26 \mathrm{~A})-\mathrm{C}(26)-\mathrm{H}(26 \mathrm{~B}) & 109.5 \\ \mathrm{C}(25)-\mathrm{C}(26)-\mathrm{H}(26 \mathrm{C}) & 109.5 \\ \mathrm{H}(26 \mathrm{~A})-\mathrm{C}(26)-\mathrm{H}(26 \mathrm{C}) & 109.5 \\ \mathrm{H}(26 \mathrm{~B})-\mathrm{C}(26)-\mathrm{H}(26 \mathrm{C}) & 109.5 \\ \mathrm{C}(25)-\mathrm{C}(27)-\mathrm{H}(27 \mathrm{~A}) & 109.5 \\ \mathrm{C}(25)-\mathrm{C}(27)-\mathrm{H}(27 \mathrm{~B}) & 109.5 \\ \mathrm{H}(27 \mathrm{~A})-\mathrm{C}(27)-\mathrm{H}(27 \mathrm{~B}) & 109.5 \\ \mathrm{C}(25)-\mathrm{C}(27)-\mathrm{H}(27 \mathrm{C}) & 109.5 \\ \mathrm{H}(27 \mathrm{~A})-\mathrm{C}(27)-\mathrm{H}(27 \mathrm{C}) & 109.5 \\ \mathrm{H}(27 \mathrm{~B})-\mathrm{C}(27)-\mathrm{H}(27 \mathrm{C}) & 109.5 \\ & \end{array}$

Symmetry transformations used to generate equivalent atoms: 
Table S3. Anisotropic displacement parameters $\left(\AA^{2} \times 10^{3}\right)$ for ed61m1s. The anisotropic displacement factor exponent takes the form: $-2 p^{2}\left[h^{2} a^{* 2} U^{11}+\ldots+2 h k a^{*} b^{*} U^{12}\right]$

\begin{tabular}{|c|c|c|c|c|c|c|}
\hline & $\mathrm{U}^{11}$ & $\mathrm{U}^{22}$ & $\mathrm{U}^{33}$ & $\mathrm{U}^{23}$ & $\mathrm{U}^{13}$ & $\mathrm{U}^{12}$ \\
\hline $\operatorname{Br}(1)$ & $24(1)$ & $41(1)$ & $33(1)$ & $-1(1)$ & $-7(1)$ & $-3(1)$ \\
\hline$S(1)$ & $19(1)$ & $22(1)$ & $19(1)$ & $0(1)$ & $2(1)$ & $-2(1)$ \\
\hline $\mathrm{O}(1)$ & $24(1)$ & $19(1)$ & $26(1)$ & $0(1)$ & $3(1)$ & $0(1)$ \\
\hline $\mathrm{C}(1)$ & $20(1)$ & $21(1)$ & $17(1)$ & $1(1)$ & $4(1)$ & $-2(1)$ \\
\hline$C(2)$ & $19(1)$ & $20(1)$ & $17(1)$ & $2(1)$ & $2(1)$ & $-1(1)$ \\
\hline$C(3)$ & $18(1)$ & $22(1)$ & $19(1)$ & $3(1)$ & $4(1)$ & $-2(1)$ \\
\hline C(4) & $20(1)$ & $25(1)$ & $20(1)$ & $2(1)$ & $5(1)$ & $-2(1)$ \\
\hline$C(5)$ & $20(1)$ & $29(1)$ & $21(1)$ & $2(1)$ & $1(1)$ & $-4(1)$ \\
\hline$C(6)$ & $19(1)$ & $33(1)$ & $26(1)$ & $7(1)$ & $5(1)$ & $3(1)$ \\
\hline$C(7)$ & $25(1)$ & $25(1)$ & $24(1)$ & $4(1)$ & $9(1)$ & $3(1)$ \\
\hline$C(8)$ & $23(1)$ & $20(1)$ & $20(1)$ & $2(1)$ & $6(1)$ & $-2(1)$ \\
\hline$C(9)$ & $24(1)$ & $22(1)$ & $20(1)$ & $0(1)$ & $3(1)$ & $0(1)$ \\
\hline$C(10)$ & $18(1)$ & $23(1)$ & $17(1)$ & $-3(1)$ & $2(1)$ & $-2(1)$ \\
\hline $\mathrm{C}(11)$ & $26(1)$ & $26(1)$ & $20(1)$ & $1(1)$ & $4(1)$ & 1(1) \\
\hline$C(12)$ & $37(2)$ & $34(2)$ & $22(1)$ & $1(1)$ & $9(1)$ & $-3(1)$ \\
\hline$C(13)$ & $33(1)$ & $41(2)$ & $26(1)$ & $-7(1)$ & $13(1)$ & $-3(1)$ \\
\hline$C(14)$ & $34(2)$ & $34(2)$ & $35(2)$ & $-9(1)$ & $12(1)$ & $7(1)$ \\
\hline$C(15)$ & $33(1)$ & $25(1)$ & $25(1)$ & $2(1)$ & $6(1)$ & $3(1)$ \\
\hline$C(16)$ & $18(1)$ & $23(1)$ & $22(1)$ & $1(1)$ & 1(1) & $-1(1)$ \\
\hline$C(17)$ & $18(1)$ & $24(1)$ & $23(1)$ & $-1(1)$ & $1(1)$ & $3(1)$ \\
\hline$C(18)$ & $24(1)$ & $26(1)$ & $30(1)$ & $-7(1)$ & $0(1)$ & $0(1)$ \\
\hline$C(19)$ & $27(1)$ & $26(1)$ & $42(2)$ & $0(1)$ & $-1(1)$ & $-9(1)$ \\
\hline$C(20)$ & $26(1)$ & $38(2)$ & $31(1)$ & $7(1)$ & $4(1)$ & $-10(1)$ \\
\hline$C(21)$ & $21(1)$ & $35(2)$ & $24(1)$ & $4(1)$ & $3(1)$ & $-5(1)$ \\
\hline$C(22)$ & $22(1)$ & $37(2)$ & $26(1)$ & $-10(1)$ & $6(1)$ & $-3(1)$ \\
\hline$C(23)$ & $37(1)$ & $52(2)$ & $44(2)$ & $-8(2)$ & $16(1)$ & $14(2)$ \\
\hline$C(24)$ & $40(2)$ & $40(2)$ & $45(2)$ & $8(1)$ & $22(2)$ & $2(1)$ \\
\hline$C(25)$ & $30(2)$ & $57(2)$ & $25(2)$ & $-5(1)$ & $12(1)$ & $-17(1)$ \\
\hline$C(26)$ & $37(2)$ & $97(3)$ & $29(2)$ & $18(2)$ & $11(1)$ & $23(2)$ \\
\hline$C(27)$ & $64(2)$ & $37(2)$ & $34(2)$ & $5(1)$ & $20(2)$ & $5(2)$ \\
\hline
\end{tabular}


Table S4. Hydrogen coordinates ( $\left.\mathrm{x} 10^{4}\right)$ and isotropic displacement parameters $\left(\AA^{2} \mathrm{x} 10^{3}\right)$ for ed61m1s.

\begin{tabular}{|c|c|c|c|c|}
\hline & $\mathrm{x}$ & $\mathrm{y}$ & $\mathrm{z}$ & $\mathrm{U}(\mathrm{eq})$ \\
\hline $\mathrm{H}(1)$ & 3762 & 3837 & 7469 & 23 \\
\hline $\mathrm{H}(2)$ & 5052 & 7995 & 7571 & 22 \\
\hline $\mathrm{H}(4)$ & 6811 & 8138 & 8741 & 26 \\
\hline $\mathrm{H}(6)$ & 9094 & 2932 & 8731 & 31 \\
\hline $\mathrm{H}(7)$ & 7752 & 874 & 7836 & 29 \\
\hline $\mathrm{H}(9 \mathrm{~A})$ & 4417 & 2401 & 6365 & 26 \\
\hline $\mathrm{H}(9 \mathrm{~B})$ & 5355 & 4415 & 6427 & 26 \\
\hline $\mathrm{H}(11)$ & 4910 & 3622 & 9022 & 29 \\
\hline $\mathrm{H}(12)$ & 3889 & 3882 & 10049 & 36 \\
\hline $\mathrm{H}(13)$ & 2751 & 7111 & 10159 & 39 \\
\hline $\mathrm{H}(14)$ & 2656 & 10122 & 9248 & 40 \\
\hline $\mathrm{H}(15)$ & 3683 & 9874 & 8220 & 33 \\
\hline $\mathrm{H}(18)$ & 1582 & 1127 & 4642 & 33 \\
\hline $\mathrm{H}(19)$ & 210 & -68 & 5358 & 39 \\
\hline $\mathrm{H}(20)$ & 57 & 1770 & 6524 & 38 \\
\hline $\mathrm{H}(22)$ & 3733 & 5416 & 5207 & 34 \\
\hline $\mathrm{H}(23 \mathrm{~A})$ & 4078 & 1403 & 4966 & 65 \\
\hline $\mathrm{H}(23 \mathrm{~B})$ & 4335 & 3063 & 4278 & 65 \\
\hline $\mathrm{H}(23 \mathrm{C})$ & 3182 & 1581 & 4164 & 65 \\
\hline $\mathrm{H}(24 \mathrm{~A})$ & 1929 & 5021 & 3883 & 60 \\
\hline $\mathrm{H}(24 \mathrm{~B})$ & 3053 & 6556 & 3905 & 60 \\
\hline $\mathrm{H}(24 \mathrm{C})$ & 2140 & 7250 & 4440 & 60 \\
\hline $\mathrm{H}(25)$ & 1817 & 6388 & 7443 & 44 \\
\hline $\mathrm{H}(26 \mathrm{~A})$ & 709 & 2304 & 7872 & 81 \\
\hline $\mathrm{H}(26 \mathrm{~B})$ & 1266 & 4281 & 8467 & 81 \\
\hline $\mathrm{H}(26 \mathrm{C})$ & 2061 & 2673 & 8037 & 81 \\
\hline $\mathrm{H}(27 \mathrm{~A})$ & -14 & 7499 & 6765 & 66 \\
\hline $\mathrm{H}(27 \mathrm{~B})$ & -10 & 7257 & 7684 & 66 \\
\hline $\mathrm{H}(27 \mathrm{C})$ & -558 & 5253 & 7098 & 66 \\
\hline
\end{tabular}


Table S5. Torsion angles $\left[{ }^{\circ}\right]$ for ed $61 \mathrm{~m} 1 \mathrm{~s}$.

\begin{tabular}{|c|c|}
\hline $\mathrm{C}(16)-\mathrm{S}(1)-\mathrm{C}(1)-\mathrm{C}(9)$ & $74.75(19)$ \\
\hline$C(16)-S(1)-C(1)-C(2)$ & $-161.87(17)$ \\
\hline $\mathrm{C}(9)-\mathrm{C}(1)-\mathrm{C}(2)-\mathrm{C}(3)$ & $-23.5(3)$ \\
\hline $\mathrm{S}(1)-\mathrm{C}(1)-\mathrm{C}(2)-\mathrm{C}(3)$ & $-145.98(17)$ \\
\hline $\mathrm{C}(9)-\mathrm{C}(1)-\mathrm{C}(2)-\mathrm{C}(10)$ & $-147.1(2)$ \\
\hline$S(1)-C(1)-C(2)-C(10)$ & $90.5(2)$ \\
\hline $\mathrm{C}(10)-\mathrm{C}(2)-\mathrm{C}(3)-\mathrm{C}(8)$ & $116.4(3)$ \\
\hline $\mathrm{C}(1)-\mathrm{C}(2)-\mathrm{C}(3)-\mathrm{C}(8)$ & $-5.4(3)$ \\
\hline $\mathrm{C}(10)-\mathrm{C}(2)-\mathrm{C}(3)-\mathrm{C}(4)$ & $-61.9(3)$ \\
\hline $\mathrm{C}(1)-\mathrm{C}(2)-\mathrm{C}(3)-\mathrm{C}(4)$ & $176.3(2)$ \\
\hline $\mathrm{C}(8)-\mathrm{C}(3)-\mathrm{C}(4)-\mathrm{C}(5)$ & $-1.1(4)$ \\
\hline $\mathrm{C}(2)-\mathrm{C}(3)-\mathrm{C}(4)-\mathrm{C}(5)$ & $177.3(2)$ \\
\hline$C(3)-C(4)-C(5)-C(6)$ & $-0.3(4)$ \\
\hline$C(3)-C(4)-C(5)-B r(1)$ & $179.17(18)$ \\
\hline$C(4)-C(5)-C(6)-C(7)$ & $1.5(4)$ \\
\hline $\mathrm{Br}(1)-\mathrm{C}(5)-\mathrm{C}(6)-\mathrm{C}(7)$ & $-177.95(19)$ \\
\hline$C(5)-C(6)-C(7)-C(8)$ & $-1.3(4)$ \\
\hline $\mathrm{C}(9)-\mathrm{O}(1)-\mathrm{C}(8)-\mathrm{C}(7)$ & $-149.4(2)$ \\
\hline $\mathrm{C}(9)-\mathrm{O}(1)-\mathrm{C}(8)-\mathrm{C}(3)$ & $31.1(3)$ \\
\hline $\mathrm{C}(6)-\mathrm{C}(7)-\mathrm{C}(8)-\mathrm{O}(1)$ & $-179.7(2)$ \\
\hline$C(6)-C(7)-C(8)-C(3)$ & $-0.2(4)$ \\
\hline $\mathrm{C}(4)-\mathrm{C}(3)-\mathrm{C}(8)-\mathrm{O}(1)$ & $-179.1(2)$ \\
\hline $\mathrm{C}(2)-\mathrm{C}(3)-\mathrm{C}(8)-\mathrm{O}(1)$ & $2.5(4)$ \\
\hline $\mathrm{C}(4)-\mathrm{C}(3)-\mathrm{C}(8)-\mathrm{C}(7)$ & $1.4(4)$ \\
\hline$C(2)-C(3)-C(8)-C(7)$ & $-177.0(2)$ \\
\hline $\mathrm{C}(8)-\mathrm{O}(1)-\mathrm{C}(9)-\mathrm{C}(1)$ & $-60.7(3)$ \\
\hline $\mathrm{C}(2)-\mathrm{C}(1)-\mathrm{C}(9)-\mathrm{O}(1)$ & $56.8(3)$ \\
\hline $\mathrm{S}(1)-\mathrm{C}(1)-\mathrm{C}(9)-\mathrm{O}(1)$ & $177.67(15)$ \\
\hline $\mathrm{C}(3)-\mathrm{C}(2)-\mathrm{C}(10)-\mathrm{C}(15)$ & $140.6(2)$ \\
\hline $\mathrm{C}(1)-\mathrm{C}(2)-\mathrm{C}(10)-\mathrm{C}(15)$ & $-96.2(3)$ \\
\hline$C(3)-C(2)-C(10)-C(11)$ & $-44.1(3)$ \\
\hline $\mathrm{C}(1)-\mathrm{C}(2)-\mathrm{C}(10)-\mathrm{C}(11)$ & $79.2(3)$ \\
\hline$C(15)-C(10)-C(11)-C(12)$ & $0.6(4)$ \\
\hline $\mathrm{C}(2)-\mathrm{C}(10)-\mathrm{C}(11)-\mathrm{C}(12)$ & $-174.9(2)$ \\
\hline
\end{tabular}




$\begin{array}{lc}\mathrm{C}(10)-\mathrm{C}(11)-\mathrm{C}(12)-\mathrm{C}(13) & 0.0(4) \\ \mathrm{C}(11)-\mathrm{C}(12)-\mathrm{C}(13)-\mathrm{C}(14) & -0.5(4) \\ \mathrm{C}(12)-\mathrm{C}(13)-\mathrm{C}(14)-\mathrm{C}(15) & 0.4(4) \\ \mathrm{C}(11)-\mathrm{C}(10)-\mathrm{C}(15)-\mathrm{C}(14) & -0.7(4) \\ \mathrm{C}(2)-\mathrm{C}(10)-\mathrm{C}(15)-\mathrm{C}(14) & 174.7(3) \\ \mathrm{C}(13)-\mathrm{C}(14)-\mathrm{C}(15)-\mathrm{C}(10) & 0.2(4) \\ \mathrm{C}(1)-\mathrm{S}(1)-\mathrm{C}(16)-\mathrm{C}(17) & -96.6(2) \\ \mathrm{C}(1)-\mathrm{S}(1)-\mathrm{C}(16)-\mathrm{C}(21) & 82.0(2) \\ \mathrm{C}(21)-\mathrm{C}(16)-\mathrm{C}(17)-\mathrm{C}(18) & -3.3(4) \\ \mathrm{S}(1)-\mathrm{C}(16)-\mathrm{C}(17)-\mathrm{C}(18) & 175.29(19) \\ \mathrm{C}(21)-\mathrm{C}(16)-\mathrm{C}(17)-\mathrm{C}(22) & 173.4(2) \\ \mathrm{S}(1)-\mathrm{C}(16)-\mathrm{C}(17)-\mathrm{C}(22) & -8.1(3) \\ \mathrm{C}(16)-\mathrm{C}(17)-\mathrm{C}(18)-\mathrm{C}(19) & 1.0(4) \\ \mathrm{C}(22)-\mathrm{C}(17)-\mathrm{C}(18)-\mathrm{C}(19) & -175.8(3) \\ \mathrm{C}(17)-\mathrm{C}(18)-\mathrm{C}(19)-\mathrm{C}(20) & 1.2(4) \\ \mathrm{C}(18)-\mathrm{C}(19)-\mathrm{C}(20)-\mathrm{C}(21) & -1.2(4) \\ \mathrm{C}(19)-\mathrm{C}(20)-\mathrm{C}(21)-\mathrm{C}(16) & -1.0(4) \\ \mathrm{C}(19)-\mathrm{C}(20)-\mathrm{C}(21)-\mathrm{C}(25) & 178.2(3) \\ \mathrm{C}(17)-\mathrm{C}(16)-\mathrm{C}(21)-\mathrm{C}(20) & 3.2(4) \\ \mathrm{S}(1)-\mathrm{C}(16)-\mathrm{C}(21)-\mathrm{C}(20) & -175.3(2) \\ \mathrm{C}(17)-\mathrm{C}(16)-\mathrm{C}(21)-\mathrm{C}(25) & -175.8(3) \\ \mathrm{S}(1)-\mathrm{C}(16)-\mathrm{C}(21)-\mathrm{C}(25) & 5.6(4) \\ \mathrm{C}(18)-\mathrm{C}(17)-\mathrm{C}(22)-\mathrm{C}(23) & -47.4(3) \\ \mathrm{C}(16)-\mathrm{C}(17)-\mathrm{C}(22)-\mathrm{C}(23) & 136.0(3) \\ \mathrm{C}(18)-\mathrm{C}(17)-\mathrm{C}(22)-\mathrm{C}(24) & 75.7(3) \\ \mathrm{C}(16)-\mathrm{C}(17)-\mathrm{C}(22)-\mathrm{C}(24) & -100.9(3) \\ \mathrm{C}(20)-\mathrm{C}(21)-\mathrm{C}(25)-\mathrm{C}(26) & 60.4(4) \\ \mathrm{C}(16)-\mathrm{C}(21)-\mathrm{C}(25)-\mathrm{C}(26) & \\ \mathrm{C}(20)-\mathrm{C}(21)-\mathrm{C}(25)-\mathrm{C}(27) & \\ \mathrm{C}(16)-\mathrm{C}(21)-\mathrm{C}(25)-\mathrm{C}(27) & \\ & \\ & \end{array}$

Symmetry transformations used to generate equivalent atoms: 


\section{References:}

(1) Bruker (2018). APEX3. Bruker AXS, Inc., Madison, Wisconsin, USA.

(2) Krause, L.; Herbst-Irmer, R.; Sheldrick, G. M.; Stalke, D. (2015) J. Appl. Cryst., 48, 3-10.

(3) Sheldrick,G.M. (2015). Acta Cryst. A71, 3-8.

(4) Sheldrick, G. M. (2015). Acta Cryst. C71, 3-8. 